\title{
Residual Dipolar Couplings: Measurements and Applications to Biomolecular Studies
}

\author{
Weidong Hu $\S *$ \\ Lincong Wang ${ }^{\dagger} \ddagger \S$
}

\begin{abstract}
Since the first successful demonstration of the tunable alignment of ubiquitin in an anisotropic liquid crystal medium only eight years ago, much progress has been made in both NMR pulse techniques for the measurements of various residual dipolar couplings (RDCs) in both proteins and nucleic acids, and applications of RDCs to many important problems in biochemistry and structural biology. In this annual report, we first review recent developments in NMR techniques for the measurements of many types of RDCs in both proteins and nucleic acids, especially a series of novel techniques for improving spectral resolution, signal to noise ratio and saving experimental time. We then describe the applications of RDCs to proteins including automated resonance assignment, structure determination, ligand-protein and proteinprotein dockings as well as protein folding.
\end{abstract}

${ }^{*}$ Immunology Division, BRI, City of Hope, Duarte Rd. 1500, Duarte, CA 91010, USA.

${ }^{\dagger}$ Dartmouth Computer Science Department, Hanover, NH 03755, USA.

${ }^{\ddagger}$ Dartmouth Chemistry Department, Hanover, NH 03755, USA.

${ }^{\S}$ Corresponding authors: Weidong Hu, Immunology Division, BRI, City of Hope, Duarte Rd. 1500, Duarte, CA 91010, USA. Email: whu@coh.org and Lincong Wang, 6211 Sudikoff Laboratory, Dartmouth Computer Science Department, Hanover, NH 03755, USA. Email: wlincong@cs . dartmouth. edu. Weidong Hu and Lincong Wang contribute equally to the work. 


\section{Introduction ${ }^{1}$}

Biological processes with the involvement of many different biomolecules are extremely complicated. Their characterization requires the use of very sophisticated experimental tools and computational techniques as well as comprehensive modelings. In this post-genomic era, biochemical research at the molecular level is focused on high-throughput determination of structures and screening of ligand-protein and protein-protein interactions. Work at the system level is focused on quantifying genetic regulatory networks and different pathways such as those involved in metabolism, signal transduction, as well as the integration of different pathways and regulatory networks. The quantitative information obtained from such researches is critical for understanding the function of a living cell or an organism as a coherent unit, and consists of the basis for developing therapeutic methods for many diseases. Solution nuclear magnetic resonance (NMR) spectroscopy, capable of exploring the electronic environments of individual nuclei, measuring a variety of geometric restraints and further monitoring the changes of these restraints with time, has become a very useful experimental tool for studying both individual biomolecules and their interactions. The electronic environments of individual nuclei can be investigated by the measurement of chemical shifts and scalar couplings. The geometric restraints measurable by NMR include internuclear distance restraints from nuclear Overhauser effect (NOE), the local angular restraints from scalar couplings, and more recently the global orientational restraints from residual dipolar couplings (RDCs). In the last decade, there are four major advances in biomolecular NMR spectroscopy that makes NMR indispensable for current biochemical research: perdeuteration [1, 2] and TROSY techniques [3] for large sized-systems, RDCs [4, 5] for measuring global orientational restraints and a series of new techniques [6] for reducing experimental time in multidimensional NMR. In this review, we focus on residual dipolar couplings: the various techniques for the measurements of RDCs as well as the ever increasing applications of RDCs to many important biochemical problems such as structure determination, protein folding, ligand-protein and protein-protein interactions. Due to the limitations of volume and our knowledge, we will not cover the application of RDCs to nucleic acids and carbohydrates. The interested readers can consult other excellent recent reviews [7, 8, 9, 10] for the application of RDCs to nucleic acids and carbohydrates [11]. The rest of the review is organized as follows. we begin with a description of the physical basis of RDCs where different forms of RDC equations are derived. The various alignment media currently available are then described very briefly, followed by a presentation of the basic NMR techniques for RDC measurement. Next, we review the recent developments in NMR pulse sequence techniques for measuring RDCs in both proteins and nucleic acids. Finally, we describe the applications of RDCs to study a series of problems in biochemistry and structural biology. Compared with several recent reviews $[12,13,14,15,16]$ on RDCs, the unique features of our review are: (a) extensive coverage of pulse sequence techniques for RDC measurement of both proteins and nucleic acids, (b) the coverage of new RDC applications such as automated chemical shift resonance assignment and protein folding.

\subsection{The physical basis of residual dipolar coupling (RDC)}

The physical basis of RDCs is the dipole-dipole (DD) interaction between two nuclear spins. The RDC equation can be derived as follows. From classical electrodynamics [17, p.101], the magnetic field $\mathbf{B}_{L}$ produced

\footnotetext{
${ }^{1}$ Abbreviations used: 2D, two-dimensional; 3D, three-dimensional; AP, anti-phase; BMRB, BioMagResBank; $\mathrm{CH}$, the vector between $\mathrm{C}_{\alpha}$ and $\mathrm{H}_{\alpha}$; CSA, chemical-shift anisotropy; CT, constant time; DD, dipole-dipole; DFS, depth-first search; ${ }^{1} \mathrm{D}_{\mathrm{NH}}$, residual dipolar coupling of internuclear vector between amide nitrogen and amide proton; E.COSY, exclusive correlation spectroscopy; FID, free-induction decay; H-bond, hydrogen bond; H-D exchange experiment, hydrogen-deuterium exchange experiment; HMQC, heteronuclear multiple quantum coherence; HSQC, heteronuclear single quantum coherence; INEPT, insensitive nuclei enhanced by polarization transfer; IP, in-phase; LC, liquid crystal; MC, Monte-Carlo; MD, molecular dynamics; MQ, multiple-quantum; NH, the internuclear vector between amide nitrogen $(\mathrm{N})$ and amide proton $(\mathrm{H})$; NMR, nuclear magnetic resonance; NOE, nuclear Overhauser effect; NOESY, nuclear Overhauser effect spectroscopy; PDB, protein data bank; POF, principal order frame; RDC, residual dipolar coupling; RMSD, root mean square deviation; $S^{3} E$, spin-state-selective excitation; $S^{3} C T$, spin-state-selective coherence transfer; SA, simulated annealing; SQ, single-quantum; TROSY, transverse relaxation optimized spectroscopy.
} 
by a spin $\mathbf{I}_{k}$ at the location $\mathbf{r}_{k l}$, far away compared with it's own dimension, is:

$$
\mathbf{B}_{L}=\frac{3\left(\mathbf{I}_{k} \mathbf{r}_{k l}\right) \mathbf{r}_{k l}-r_{k l}^{2} \mathbf{I}_{k}}{r_{k l}^{5}}
$$

where we have ignored the physical constants. The dipole-dipole interaction Hamiltonian, $\mathscr{H}_{D}$, between two spins $\mathbf{I}_{k}$ and $\mathbf{I}_{l}$ is

$$
\mathscr{H}_{D}=-\mathbf{B}_{L} \mathbf{I}_{l}=r_{k l}^{-3}\left(\mathbf{I}_{k} \mathbf{I}_{l}-3 \frac{1}{r_{k l}^{2}}\left(\mathbf{I}_{k} \mathbf{r}_{k l}\right)\left(\mathbf{I}_{l} \mathbf{r}_{k l}\right)\right)
$$

Eq. (2) can be represented in tensor form in order to separate the spin and space terms,

$$
\mathscr{H}_{D}=r_{k l}^{-3} \sum_{q=-2}^{2} F_{k l}^{(q)} A_{k l}^{(q)}
$$

where both $F_{k l}$ and $A_{k l}$ are second rank tensors:

$$
\begin{aligned}
A_{k l}^{(0)} & =I_{k z} I_{l z}-\frac{1}{4}\left(I_{k}^{+} I_{l}^{-}+I_{k}^{-} I_{l}^{+}\right), & F_{k l}^{(0)} & =1-3 \cos ^{2} \theta_{k l}, \\
A_{k l}^{(1)} & =-\frac{3}{2}\left(I_{k z} I_{l}^{+}+I_{k}^{+} I_{l z}\right), & F_{k l}^{(1)} & =\sin \theta_{k l} \cos \theta_{k l} e^{-i \phi_{k l}}, \\
A_{k l}^{(-1)} & =-\frac{3}{2}\left(I_{k z} I_{l}^{-}+I_{k}^{-} I_{l z}\right), & F_{k l}^{(-1)} & =\sin \theta_{k l} \cos \theta_{k l} e^{+i \phi_{k l}}, \\
A_{k l}^{(2)} & =-\frac{3}{4} I_{k}^{+} I_{l}^{+}, & F_{k l}^{(2)} & =\sin ^{2} \theta_{k l} e^{-2 i \phi_{k l}}, \\
A_{k l}^{(-2)} & =-\frac{3}{4} I_{k}^{-} I_{l}^{-}, & F_{k l}^{(-2)} & =\sin ^{2} \theta_{k l} e^{+2 i \phi_{k l}}
\end{aligned}
$$

where $\theta_{k l}$ and $\phi_{k l}$ are polar angles specifying the orientation of the internuclear vector $\mathbf{r}_{k l}$ in a frame with the external magnetic field $\mathbf{B}_{0}$ in the $+z$-axis (laboratory frame). In the high field approximation, only the $q=0$ term of $A_{k l}$ contributes (all other terms account for less than $2 \%$ at $500 \mathrm{MHz}$ ) so we have

$$
\mathscr{H}_{D}^{\text {trunc }}=\frac{3}{2 r_{k l}^{3}}\left(1-3 \cos ^{2} \theta_{k l}\right)\left[3 I_{k z} I_{l z}-I_{k}^{+} I_{l}^{-}+I_{k}^{-} I_{l}^{+}\right]
$$

In heteronuclear spin systems (e.g. $I_{k}=$ proton and $S_{l}=$ nitrogen) we can further approximate the Hamiltonian as;

$$
\mathscr{H}_{D}^{I S}=\frac{3}{2 r_{k l}^{3}}\left(1-3 \cos ^{2} \theta_{k l}\right) I_{k z} I_{l z}
$$

This approximation is good for both solid and solution state NMR. If the tensor $F_{k l}$ is also represented in Cartesian coordinates, its expressions will be identical to those for $A_{k l}$. From a mathematical viewpoint, we can draw a similarity between spins in spin space and points in real space. The basis for such a similarity (Eq. (3)) can be directly traced to group representation of angular moments and spins. The two tensors form the basis for the representation of rotation groups in their respective spaces (spin space and real 3D coordinate space). Eq. (5) is also the basis for using the experiments for measuring scalar coupling to measure RDCs since the RDC Hamiltonians have the same spin terms as those for scalar couplings.

In order to describe dipole-dipole interactions in solution NMR experiments, we must resort to statistical mechanics since the signals observed in an NMR experiment are averages over an ensemble of molecules such as proteins. In solution, the solute (target) molecules tumble very fast, so that the average dipole-dipole interaction becomes zero. However, it is a mistake to assume that dipole-dipole interactions do not exist in an isotropic solution. In fact, only the first-order term, which is proportional to $\frac{1}{r^{3}}$, of an expansion for the 
evolution of an NMR observable such as an $I_{z}$ spin operator under the dipole-dipole interaction Hamiltonian $\mathscr{H}_{D}$, becomes zero. The second-order term of the same expansion is non-zero and proportional to $\frac{1}{r^{6}}$, which can be observed in an NOE experiment $[18,19]$. When solute molecules such as proteins are weakly aligned with an external magnetic field either by the intrinsic anisotropic magnetic susceptibility of the solutes or by putting the solutes in a medium such as a dilute liquid crystal (LC) medium, the first order term is no longer zero. What remains is called the residual dipolar coupling (RDC). Thus, the equation for RDC Hamiltonian, Eq. (5), becomes [20]:

$$
\mathscr{H}_{D}^{I S}=\frac{3}{2 r_{k l}^{3}}\left\langle 1-3 \cos ^{2} \theta_{k l}\right\rangle I_{k z} I_{l z}
$$

where the brackets denote the ensemble average. If we choose a molecular frame in which the direction of $\mathbf{B}_{0}$ is specified by three angles $\theta_{x^{\prime}}, \theta_{y^{\prime}}$ and $\theta_{z^{\prime}}$, and the direction of $\mathbf{r}_{k l}$ by three angles $\phi_{x^{\prime}}, \phi_{y^{\prime}}$ and $\phi_{z^{\prime}}$, then Eq. (6) becomes

$$
\mathscr{H}_{D}^{I S}=\frac{3}{2 r_{k l}^{3}}\left\langle\sum_{i, j} \cos \phi_{i} S_{i, j} \cos \phi_{j}\right\rangle I_{k z} I_{l z}
$$

where $S_{i, j}=\frac{3 \cos \theta_{i} \cos \theta_{j}-\delta_{i, j}}{2}, i, j=x^{\prime}, y^{\prime}, z^{\prime}$ and $\delta_{i, j}$ is the Kronecker delta. The matrix $\mathbf{S}$ is a $3 \times 3$ traceless and symmetric matrix called the Saupe order matrix, or alignment tensor, or order tensor. The matrix $\mathbf{S}$ specifies the ensemble-averaged anisotropic orientation of a molecule in the laboratory frame. Assuming that the overall rotation of the entire molecule can be decoupled from the internal motions of its individual internuclear vectors, Eq. (7) becomes

$$
\mathscr{H}_{D}^{I S}=\frac{3}{2 r_{k l}^{3}} \sum_{i, j} S_{i, j}\left\langle\cos \phi_{i} \cos \phi_{j}\right\rangle I_{k z} I_{l z}
$$

The ensemble average over the internal motions, $\left\langle\cos \phi_{i} \cos \phi_{j}\right\rangle$, can be described in different levels of detail. Similar to the Saupe matrix used to describe the overall anisotropic motion of the entire molecule, the most detailed representation of the anisotropic internal motion for an internuclear vector relevant to RDCs is a $3 \times 3$ symmetric matrix with five independent elements. Such a representation gives of the basis for extracting, simultaneously, the structural and dynamic information from RDCs measured in multiple media [21, 22, 23].

In the simplest case, the internal motion can be described by a single variable, order parameter $S^{2}$, then Eq. (8) becomes

$$
\mathscr{H}_{D}^{I S}=\frac{3}{2 r_{k l}^{3}} S^{2} \sum_{i, j} \cos \phi_{i} S_{i, j} \cos \phi_{j} I_{k z} I_{l z}
$$

The matrix $\mathbf{S}$ can be diagonalized, and in that frame $\mathbf{S}$, Eq. (9) becomes

$$
\mathscr{H}_{D}^{I S}=\frac{3 S^{2}}{2 r_{k l}^{3}}\left(S_{x x} x^{2}+S_{y y} y^{2}+S_{z z} z^{2}\right) I_{k z} I_{l z}
$$

where $S_{x x}, S_{y y}$ and $S_{z z}$ are the three diagonal elements of a diagonalized Saupe matrix $\mathbf{S} . x=\cos \phi_{x}, y=$ $\cos \phi_{y}$ and $z=\cos \phi_{z}$ are, respectively, the $x, y$ and $z$-components of an internuclear unit vector $\mathbf{v}=(x, y, z)$ in the principal order frame (POF) which diagonalizes $\mathbf{S}$. Since diagonalizing a $3 \times 3$ matrix is equivalent to a rotation in the $3 \mathrm{D}$ space, three Euler angles can be used to specify the relative rotation between a POF and any molecular-fixed frame. Finally, since RDCs are usually measured in NMR experiments by following the changes in either scalar couplings or the modulation of peak intensities by scalar couplings, the equation for a RDC $r$ is generally written with only the space terms while the spin term $\left(I_{k z} I_{l z}\right)$ is ignored. The RDC equation can be written as

$$
r=D_{\max } S^{2}\left(S_{x x} x^{2}+S_{y y} y^{2}+S_{z z} z^{2}\right), \quad D_{\max }=\frac{\mu_{0} \hbar \gamma_{N} \gamma_{H}}{4 \pi r_{k l}^{3}}
$$


where we have introduced the physical constants back into the equation. Note that $x^{2}+y^{2}+z^{2}=1$ and $S_{x x}+S_{y y}+S_{z z}=0$. Thus, given $\mathbf{S}$, Eq. (11) represents an ellipse on a 2-sphere, and the RDC data constrains onto the ellipse the orientation of the unit vector $\mathbf{v}$ with respect to a POF common to all the RDCs measured on the same aligned biomolecule. In other words, extracting global orientation restraints on internuclear vectors from scalar RDC data requires the Saupe matrix $\mathbf{S}$ to be known a priori. Another RDC equation, which is equivalent to Eq. (11) but use polar angles $\theta$ and $\phi$ to specify the direction of $\mathbf{v}$ is

$$
r=D_{\max } S^{2} D_{a}\left(\left(3 \cos ^{2} \theta-1\right)+R \frac{3 \sin ^{2} \theta \cos 2 \phi}{2}\right)
$$

where $D_{a}=\frac{S_{Z Z}}{3}$ and $R=\frac{S_{x x}-S_{y y}}{S_{Z Z}}$ are called, respectively, axial and rhombicity alignment parameters. Another variable called $D_{r}$ defined as $D_{r}=R D_{a}$, also appears frequently in the literature. In most applications, the order of parameter $S^{2}$ in Eqs. (9-12) is assumed to be 1.

\subsection{Alignment media}

Since the first successful demonstration of the alignment of ubiquitin in lipid bicelles [4], many types of alignment media have been developed for the following reasons:

1. Some alignment media may not be suitable for the target solute (biomolecule) due to unwanted interactions, such as electrostatic interactions between the solute and alignment media, which can increase the total correlation time for the target molecules;

2. Some alignment media may be incompatible with certain sample conditions such as $\mathrm{pH}$, ionic strength and temperature etc.;

3. More than one alignment media is needed to compute more precise structures [24, 25] and quantify dynamics $[21,22]$.

The following is a list of recently discovered alignment media: cetylpyridinium-based media [26, 27], filamentous phage [28, 29], purple membrane fragments [30], cellulose crystallites [31], compressed polyacrylamide gel [32], alkyl poly(ethylene glycol) based media [33], strained acrylamide/acrylate copolymers [34], and embedded filamentous Pf1 phage in polyacrylamide matrix [35]. Nice summaries of the properties of alignment media and how to prepare oriented samples with different alignment media can be found in several recent reviews $[36,37,11]$.

\section{The basic techniques for measuring residual dipolar couplings}

The experiments for measuring RDCs are based on two basic techniques: frequency-displacement techniques, where RDCs are measured directly by following changes in J-couplings (for example, the difference in ${ }^{1} \mathbf{J}_{\mathrm{NH}}$ coupling as measured on a partially oriented sample and the same ${ }^{1} \mathbf{J}_{\mathrm{NH}}$ coupling as measured on an isotropic sample), and quantitative J-modulation techniques, where the couplings are obtained from variations in peak intensities induced by both the J-couplings and RDCs. The frequency-displacement techniques can be further classified into three distinct categories:

1. Direct measurement of the resolved J couplings from heteronuclear edited spectra for small or mediumsized proteins;

2. Spin-state-separation techniques such as the IPAP experiment [38], $S^{3} E[39,40]$ and $S^{3} C T$ scheme [41] to resolve crowded spectra;

3. E.COSY-type [42] experiments to resolve small couplings between a pair of spins through a larger coupling between another pair of spins.

In addition, the TROSY scheme [3] has been widely used in RDC measurement for large-sized biomolecules. In the following, we will first discuss the principles and pros and cons of these commonly used techniques. 


\subsection{J-resolved experiment}

The J-resolved experiment is the most straightforward approach to coupling measurement. It is particularly useful for measuring large one-bond heteronuclear couplings such as ${ }^{13} \mathrm{C}_{\alpha^{-}}{ }^{1} \mathrm{H}_{\alpha}$ and ${ }^{1} \mathrm{H}^{-15} \mathrm{~N}$ couplings in proteins. In 2D heteronuclear correlation experiments, the couplings can evolve in either $t_{1}$ or $t_{2}$ dimension, and the cross peaks are split into doublets for isolated spin pairs such as ${ }^{13} \mathrm{C}_{\alpha^{-}}{ }^{1} \mathrm{H}_{\alpha}$ or ${ }^{1} \mathrm{H}^{-15} \mathrm{~N}$. The couplings can be extracted from the split separation. Since it is frequency-based approach, measurement accuracy is less likely to be affected by factors that affect the peak intensity. The peak separation should be large compared to the linewidth in order to avoid potential measurement errors. The approach works well for small-sized biomolecules. For medium- and large-sized proteins, the coupled spectrum becomes crowded, and consequently makes it very difficult to measure a complete or near-complete set of couplings. To overcome this problem, spin-state-selection (see Section 2.2) can be used to separate doublets into two sub-spectra. Alternatively, the $2 \mathrm{D}$ experiment can be extended to $3 \mathrm{D}$ to enhance the resolution, such as the measurement of $\mathrm{C}_{\alpha}-\mathrm{H}_{\alpha}$ coupling with $3 \mathrm{D}^{1} \mathrm{~J}_{\mathrm{C}_{\alpha} \mathrm{H}_{\alpha}}$-resolved (HA)CACONH [43] and 3D HA(CA)CONH [44] experiments.

\subsection{Spin-state-selection technique}

Spin-state-selection is a technique developed to reduce the overlap of coupled spectra obtained from J-resolved experiments. It separates the two components of a doublet in a J-resolved experiment into two different spectra, where one spectrum corresponds to the $\alpha$ spin state of the coupled spin and the other spectrum the $\beta$ state. The spectral resolution is thus enhanced significantly. More importantly, small couplings not accessible directly via the J-resolved method become measurable because the individual peaks of doublets are now separated into two different spectra. There are several approaches to achieving this goal [45, 39, 40, 38, 41]. In the following, we focus on IPAP, $\mathrm{S}^{3} \mathrm{E}$ and $\mathrm{S}^{3} \mathrm{CT}$ schemes because they are most frequently used in current experiments.

IPAP-HSQC experiment: The experiment is carried out by acquiring two date sets in an interleaved manner [38]. The first experiment is a regular HSQC with ${ }^{1} \mathrm{H}$ coupled to ${ }^{15} \mathrm{~N}$ during $t_{1}$ evolution. The ${ }^{15} \mathrm{~N}$ chemical shifts are modulated by $\cos \left(\mathrm{J}_{\mathrm{HN}} \mathrm{t}_{1}\right)$, and the doublets have the same sign. The spectrum is called in-phase (IP). In the second experiment, an extra INEPT step (the block within the rectangular box in Fig. 1a) is used to transfer the ${ }^{15} \mathrm{~N}$ anti-phase term to an in-phase term prior to the ${ }^{15} \mathrm{~N}$ evolution. After the ${ }^{15} \mathrm{~N}$ evolution period, only the term modulated by $\sin \left(\mathrm{J}_{\mathrm{HN}} \mathrm{t}_{1}\right)$ is detected, and the doublets have the opposite sign. The spectrum is called anti-phase (AP). The $90^{\circ}$ purge pulse on ${ }^{1} \mathrm{H}$ prior to the $t_{1}$ period is used to transfer the remaining residual $\cos \left(\pi \mathrm{J}_{\mathrm{HN}} \Delta\right) \mathrm{H}_{\mathrm{Z}} \mathrm{N}_{\mathrm{x}}$ to unobservable multiple quantum terms. A more detailed description in product operator formalism, such as how to obtain the IP and AP spectra, can be found in the original paper [38]. The addition and subtraction of the IP and AP spectra give two simplified spectra just like regular HSQCs with the

${ }^{15} \mathrm{~N}$ chemical shifts displaced by either $+\mathrm{J}_{\mathrm{NH}} / 2$ or $-\mathrm{J}_{\mathrm{NH}} / 2$ as schematically shown in Fig. 2 . The couplings can be readily measured from the frequency difference of the corresponding peaks in the two spectra.

$\mathbf{S}^{3} \mathbf{E}$ element: $\quad$ Fig. $1 b$ shows the basic pulse scheme for spin-state-selective excitation $\left(\mathrm{S}^{3} \mathrm{E}\right)[39,40]$. The $\mathrm{S}^{3} \mathrm{E}$ can be easily understood using product operator formalism. Just before the second $90^{\circ}$ pulse on spin I, two terms evolve from magnetization I due to $\mathrm{J}_{\mathrm{IS}}$ evolution: $\mathrm{I}_{\mathrm{y}} \cos \left(\pi \mathrm{J}_{\mathrm{IS}} \tau\right)+2 \mathrm{I}_{\mathrm{x}} \mathrm{S}_{\mathrm{z}} \sin \left(\pi \mathrm{J}_{\mathrm{IS}} \tau\right)$. The terms have equal intensities if $\tau$ is set to $\frac{1}{4} \mathrm{~J}_{\text {IS }}$. The selective observation of either one of the two terms can be achieved by phase cycling of $\phi_{3}$ and $\phi_{4}$ of the last two $90^{\circ}$ pulses on spin I. The two data sets are stored separately, and then the addition and subtraction separate the upfield and downfield components of doublets I- $\{\mathrm{S}\}$ into two sub-spectra. An $S^{3} E$ element is usually applied in the preparation period of the pulse sequence.

$S^{3}$ CT element: Spin-state-selective coherence transfer $\left(S^{3} \mathrm{CT}\right)$ [41] combines coherence transfer with spinstate-selection. The basic function of this unit is to transfer double quantum (DQ) or zero-quantum (ZQ) terms of a coupled I-S spin pair to a desired single quantum (SQ) term. The collective effect of the pulses shown in 
(a)

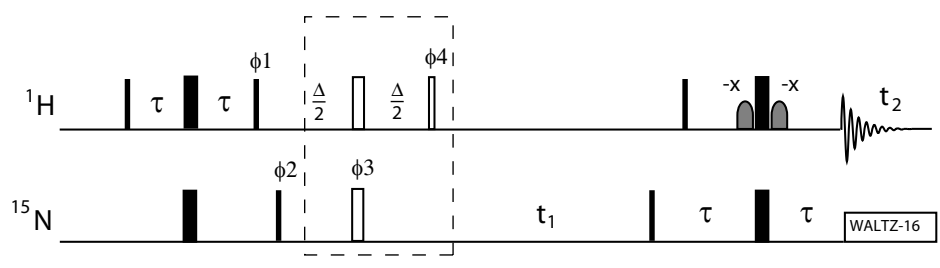

(b)

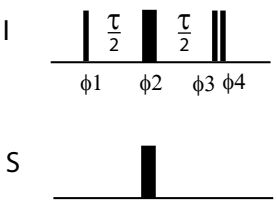

(c)

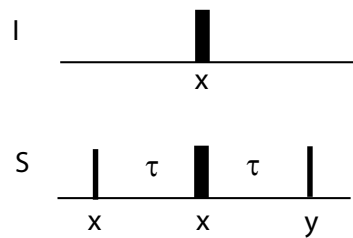

Figure 1: Basic spin-state-selection elements used in experiments for coupling measurements. a) IPAPHSQC, b) $\mathrm{S}^{3} \mathrm{E}$ and c) $\mathrm{S}^{3} \mathrm{CT}$. The phases of all the pulses are on $x$ unless indicated otherwise. The narrow and wide bars stand for $90^{\circ}$ and $180^{\circ}$ pulses, respectively. $\tau$ is set to $\frac{1}{4 \mathrm{~J}_{\mathrm{NH}}}$ in a), $\frac{1}{4 \mathrm{~J}_{\mathrm{IS}}}$ in b) and c). $\Delta$ is set to $\frac{1}{2 \mathrm{~J}_{\mathrm{NH}}}$ in a). More technical details can be found in the original papers [38] for a), $[39,40]$ for $b$ ), and [41] for c).

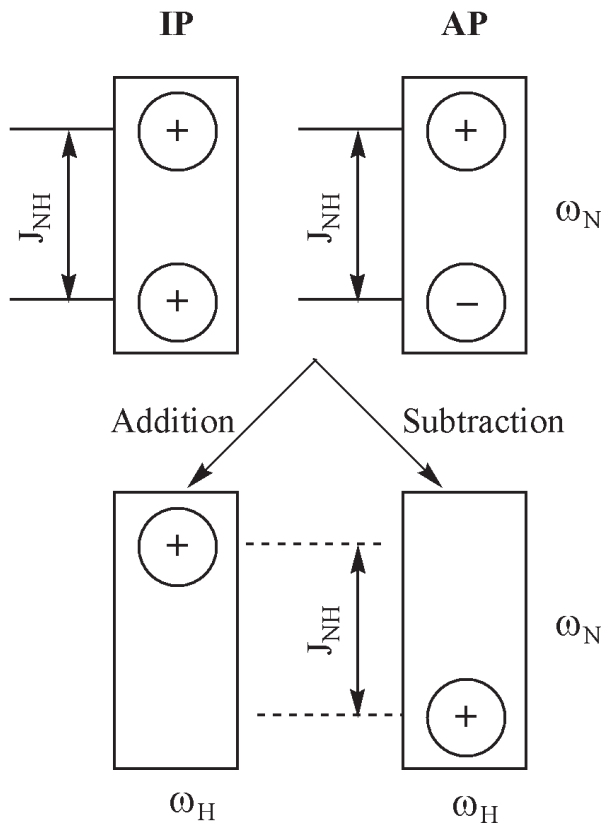

Figure 2: Schematic illustration of a procedure for extracting ${ }^{1} \mathbf{H}-{ }^{15} \mathbf{N}$ couplings from an IPAP-HSQC experiment. 
Fig. $1 \mathrm{c}$ is equivalent to a $\pi \mathrm{I}^{\alpha} \mathrm{S}_{y}$. Thus the $\alpha$ and $\beta$ states of the $\mathrm{S}$ nucleus exchange only if they are coupled to $\mathrm{I}^{\alpha}$, but not $\mathrm{I}^{\beta}$. These can be expressed using product operator formalism as follows:

$$
\begin{array}{lll}
I^{-} S^{-} & \stackrel{\pi I^{\alpha} S_{y}}{\longrightarrow} & I^{-} S^{\beta} \\
I^{-} S^{+} & \stackrel{\pi I^{\alpha} S_{y}}{\longrightarrow} & -I^{-} S^{\alpha} \\
I^{+} S^{-} & \stackrel{\pi I^{\alpha} S_{y}}{\longrightarrow} & -I^{+} S^{\alpha} \\
I^{+} S^{+} & \stackrel{\pi I^{\alpha} S_{y}}{\longrightarrow} & I^{+} S^{\beta}
\end{array}
$$

Through phase cycling, the coherence transfer from DQ to $\mathrm{I}^{-} \mathrm{S}^{\beta}$ and $\mathrm{I}^{+} \mathrm{S}^{\beta}$ can be separated from the coherence transfer from ZQ to $\mathrm{I}^{-} \mathrm{S}^{\alpha}$ and $\mathrm{I}^{+} \mathrm{S}^{\alpha}$, and the coherence of I spin coupled to either $\alpha$ or $\beta$ state of $\mathrm{S}$ spin can be selected.

The efficiency of spin-state-selection can be affected by (a) differential relaxation rates of the consisting components due to the cross-correlation of chemical shift anisotropy (CSA) and DD, (b) pulse imperfections in the spin-state-filter elements and (c) J-mismatch. A J-mismatch happens when the value of a measured RDC $+\mathrm{J}$ is different from the value used in the spin-state-selection element. $\mathrm{J}$-mismatch results in phase distortion for the observed peaks, although this can be removed by purge pulses [38]. In addition, J-mismatch causes the intensities of the in- and anti-phase terms to become unequal, and thus makes unwanted components appear in the spectrum of selected components, the so-called J-crosstalk. Among the three spin-state-selection elements, IPAP and $S^{3} \mathrm{CT}$ have better tolerance than $\mathrm{S}^{3} \mathrm{E}$ for J-mismatch. To remove or suppress the J-crosstalk, a scaling factor has been used when the in-phase and anti-phase data sets are combined linearly [38, 46]. A more recently proposed scheme claims to better suppress the J-mismatch [47].

\subsection{E.COSY method}

Exclusive correlation spectroscopy (E.COSY) was originally developed to enhance the resolution of experiments measuring ${ }^{1} \mathrm{H}-{ }^{1} \mathrm{H}$ couplings [42]. It requires an A-M-X three coupled spin system to work, making use of the larger $\mathrm{J}_{\mathrm{MX}}$ coupling to resolve the smaller $\mathrm{J}_{\mathrm{AX}}$ coupling in an $[\mathrm{A}, \mathrm{M}]$ correlated experiment. The spin $\mathrm{X}$ is called a passive spin, whose $\alpha$ and $\beta$ states are not mixed between the detection of $\mathrm{A}$ and $\mathrm{M}$ spins. E.COSY's application to heteronuclear coupling measurement was first demonstrated in a 2D HNCA-J experiment [48], which made use of large ${ }^{1} \mathrm{H}-{ }^{13} \mathrm{C}_{\alpha}$ couplings to resolve the small ${ }^{3} \mathrm{~J}_{\mathrm{HN}-\mathrm{H} \alpha}$ couplings. For ease of exposition, a $2 \mathrm{D}\left[{ }^{15} \mathrm{~N},{ }^{1} \mathrm{H}\right]-\mathrm{HSQC}$ is used as an example. If the experiment is carried out without perturbing the spin-state of $\mathrm{C}^{\prime}$ on a small peptide, then the regular cross peaks become doublets due to the coupling to $\mathrm{C}^{\prime}$ in both ${ }^{15} \mathrm{~N}$ and ${ }^{1} \mathrm{H}$ dimensions. One component of the doublet is coupled to $\mathrm{C}^{\prime}(\alpha)$ and can be described as $\left[\cos \left(\omega_{\mathrm{N}}+\pi^{1} \mathrm{~J}_{\mathrm{NC}^{\prime}}\right) \mathrm{t}_{1}\right] \exp \left[\mathrm{i}\left(\omega_{\mathrm{HN}}+\pi^{2} \mathrm{~J}_{\mathrm{HN}-\mathrm{C}^{\prime}}\right) \mathrm{t}_{2}\right]$ while the other component is coupled to $\mathrm{C}^{\prime}(\beta)$ and can be represented as $\left[\cos \left(\omega_{\mathrm{N}}-\pi^{1} \mathrm{~J}_{\mathrm{NC}^{\prime}}\right) \mathrm{t}_{1}\right] \exp \left[\mathrm{i}\left(\omega_{\mathrm{HN}}-\pi^{2} \mathrm{~J}_{\mathrm{HN}-\mathrm{C}^{\prime}}\right) \mathrm{t}_{2}\right]$. An E.COSY pattern with two cross peaks is shown schematically in Fig. 3. Although the small coupling of ${ }^{2} \mathrm{~J}_{\mathrm{HN}-\mathrm{C}^{\prime}}$ is not resolved along the ${ }^{1} \mathrm{H}$ dimension, it can now be measured from the two cross peaks because they are well resolved along the ${ }^{15} \mathrm{~N}$ dimension. One advantage of the E.COSY method is that the slope of two cross peaks reflects the relative sign of two measured couplings as shown in the two trigonometry terms given above. Basically, if the two couplings have the same sign, then the direction of the line connecting two cross peaks goes from the lower left to the upper right; otherwise, the direction of the line goes from the lower right to the upper left. Please note that the sign of slope can be reversed if there is a $180^{\circ}$ pulse issued on the passive spin between two coupling evolution periods.

To form an E.COSY pattern, the $\alpha$ - and $\beta$-states of passive nucleus should not be mixed. $\mathrm{T}_{1}$ relaxation of passive spin between the two coupling evolution periods and during the data acquisition may mix the two spin states, reducing the separation of E.COSY doublets. Thus, E.COSY methods may underestimate coupling values. This is not a big problem if the passive spin is a ${ }^{13} \mathrm{C}$ or ${ }^{15} \mathrm{~N}$ because their $\mathrm{T}_{1}$ values are usually long compared to experimental time scales. However, caution should be taken if the passive spin is a proton. 


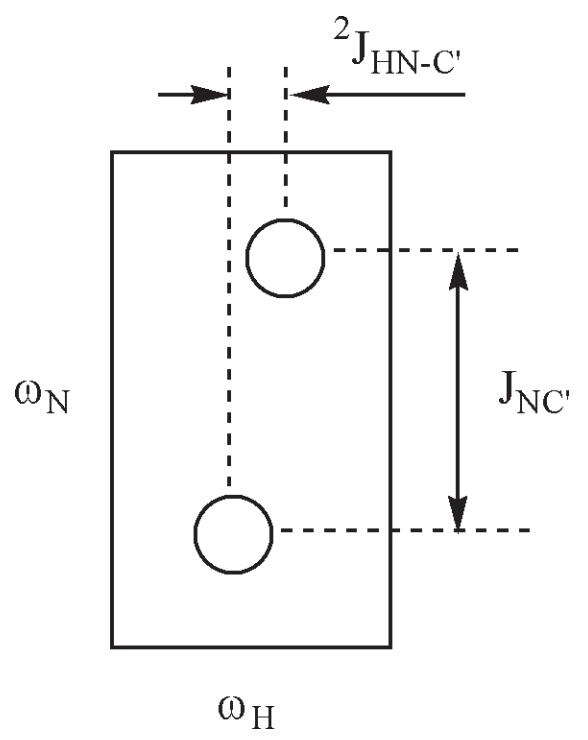

Figure 3: E.COSY pattern. Schematic illustration of doublet obtained from an $\left[{ }^{15} \mathrm{~N},{ }^{1} \mathrm{H}\right]-\mathrm{HSQC}$ experiment without $\mathrm{C}^{\prime}$ decoupling for both ${ }^{15} \mathrm{~N}$ and ${ }^{1} \mathrm{H}$ dimensions. Doublet are well resolved along the ${ }^{15} \mathrm{~N}$ dimension due to $\mathrm{J}_{\mathrm{NC}^{\prime}}$ coupling, thus the small coupling ${ }^{2} \mathrm{~J}_{\mathrm{HN}-\mathrm{C}^{\prime}}$ can be measured from doublet along the ${ }^{1} \mathrm{H}$ dimension.

\subsection{Quantitative J correlation experiment}

The experiment is based on the observation that NMR signal intensities can be modulated by coupling evolution. There are several different ways to implement this idea [49]. The first approach is to acquire two data sets where the signal is modulated with a cosine function in one experiment, but not modulated in the other socalled reference experiment. The coupling can then be derived from the intensity ratio of the two experiments. A second approach is to run a series of experiments where the signal is modulated by an array of time delays where couplings are active. The coupling constants can then be extracted from a curve fitting of the observed peak intensities versus the coupling evolution time [50]. This approach usually offers higher precision than the first one. A third approach is to determine the couplings from a single spectrum where the intensity ratio of cross and diagonal peaks is a well defined function of the magnitude of targeted couplings [51, 52].

\subsection{TROSY experiment}

Transverse relaxation optimized spectroscopy (TROSY) is a powerful experiment for studying large-sized biomolecules [3]. It makes use of cross-correlation interference between CSA and DD interactions and selects only the narrowest component out of four coupled multiplets. For an isolated coupled spin- $\frac{1}{2}$ system, I-S, in a protein, the $\mathrm{T}_{2}$ relaxation rates of both spins are dominated by the DD interaction between I and $\mathrm{S}$ as well as the CSA of each spin. To explain how TROSY works, we can take a look at the $\mathrm{T}_{2}$ relaxation of I spin. The CSA of I spin contributes equally to $\mathrm{T}_{2}$ relaxation of $\mathrm{I}^{-} \mathrm{S}^{\alpha}$ and $\mathrm{I}^{-} \mathrm{S}^{\beta}$ transitions (single quantum transitions), while the DD contributions to $\mathrm{T}_{2}$ relaxation of $\mathrm{I}^{-} \mathrm{S}^{\alpha}$ and $\mathrm{I}^{-} \mathrm{S}^{\beta}$ transitions have opposite sign. Thus, the contributions of CSA and DD add up for $\mathrm{I}^{-} \mathrm{S}^{\alpha}$ transition, and counteract for the $\mathrm{I}^{-} \mathrm{S}^{\beta}$ transition. The same phenomenon also holds true for the relaxation rates of $S$ spin single quantum transitions. When two interactions are collinear and their magnitudes are comparable, the $T_{2}$ relaxation rates of $\mathrm{I}^{-} \mathrm{S}^{\beta}$ and $\mathrm{S}^{-} \mathrm{I}^{\beta}$ can be significantly reduced. In particular, since CSA is proportional to the field strength, the CSA and DD interactions are almost canceled for an amide spin pair, ${ }^{1} \mathrm{H}-{ }^{15} \mathrm{~N}$, when the magnetic field strength corresponding to a ${ }^{1} \mathrm{H}$ frequency is at $1.1 \mathrm{GHz}$ [3]. Experimentally, the $\left[{ }^{1} \mathrm{H},{ }^{15} \mathrm{~N}\right]$-TROSY sequence chooses only the transition $\mathrm{N}^{ \pm} \mathrm{H}^{\beta} \rightarrow \mathrm{H}^{\mp} \mathrm{N}^{\beta}$ through a combination of proper phase cycling and echo/anti-echo quadrature detection [53]. A schematic 


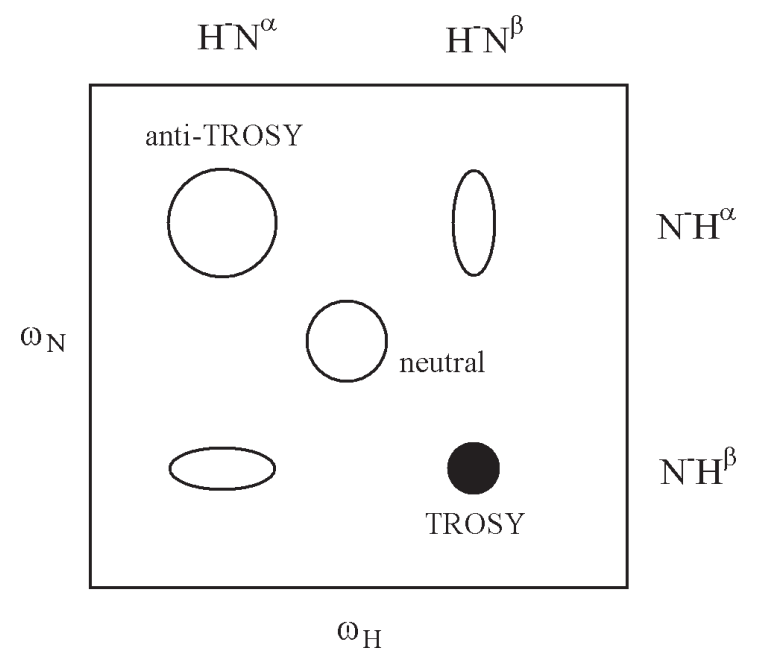

Figure 4: Schematic multiplets of a coupled ${ }^{15} \mathbf{N}-{ }^{1} \mathrm{H}$ spin pair with TROSY effect. The diameters of circles, and the major and minor axes of ellipses reflect the linewidth of each component. The components coupled with partner's $\beta$ states have narrower linewidth. TROSY experiment only observes the narrowest component (black circle). The broadest one is called anti-TROSY peak [55]. The middle neutral component represents a peak from a regular decoupled HSQC.

illustration of the TROSY effect of a coupled ${ }^{15} \mathrm{~N}-{ }^{1} \mathrm{H}$ spin pair is shown in Fig. 4.

The TROSY method makes it much easier to use NMR spectroscopy to study large-sized proteins (up to $100 \mathrm{kDa}$ ) [54] by providing optimal sensitivity and resolution. Specifically, the TROSY scheme can be easily implemented in all kinds of pulse sequences for coupling measurement (see Section 3).

However, before going into a detailed discussion of the applications of these basic methods to RDC measurement, we will first consider the measurement precision and sensitivity.

\subsection{General considerations for measurement precision and sensitivity}

Before the RDCs of partially ordered protein samples prepared using a variety of alignment media (Section 1.2) can be measured successfully, they can only be obtained from the magnetic field-dependent alignment $[36,56,57]$. Such RDCs are rather small, ranging from a few Hertz for a system with large magnetic susceptibility anisotropy [57], to as small as a few tenths of a Hertz for diamagnetic protein samples [50]. In order to measure such small RDCs accurately, several different versions of $\left[{ }^{15} \mathrm{~N},{ }^{1} \mathrm{H}\right]$-HSQC experiments have been designed based on either frequency displacement or J-quantitative modulation approaches [50, 51, 58]. The precisions of these experiments range from $0.5 \mathrm{~Hz}$ to $0.02 \mathrm{~Hz}$.

In practice, the relatively large uncertainty in RDC values (measurement error vs. measurement value) obtained from field aligned samples severely limit the application of RDCs to structural and dynamic studies of biomolecules in solution. The relative error was reduced by more than an order of a magnitude by Bax and Tjandra [59, 4], who adopted the liquid crystal (LC) medium, used in LC NMR to align small organic molecules [20], to align proteins to an extent that balances the line broadening with the increase in RDC values. The LC medium (bicelles) can be easily made from a mixture of dihexanoyl phosphatidylcholine (DHPC) and dimyristoyl phosphatidylcholine (DMPC) in water, and the concentration of bicelles can range from 3\% to $10 \% \mathrm{w} / \mathrm{v}$. The method provides a tunable ordering of the solutes (proteins), and the dipolar contribution to the ${ }^{1} \mathrm{H}_{-}{ }^{15} \mathrm{~N}$ splitting is proportional to the bicelle concentration. The RDC magnitude obtained on ubiquitin in 
such bicelle media is about 2 orders larger than that obtained in field dependent alignment [50].

As is well known in LC NMR for small molecules, increasing the ordering enlarges not only the RDCs for the specific pairs of interest, but all the RDCs, including those between two protons. The latter will significantly broaden the linewidths of NMR signals and lead to a reduction in both the resolution and sensitivity of the signals. As the size of the molecules being studied increases, the resolution and sensitivity deteriorate quickly, and this is a major force for the invention of many new pulse schemes for measuring RDCs.

Generally, quantitative J-modulation experiments can deliver higher measurement precision than frequencydisplacement ones. However, in the J-modulation method systematic errors [60] may occur, caused by differential relaxation properties of the two components (due to DD-CSA cross correlation, DD-DD cross correlation), coupling to a third spin, or pulse imperfection etc. Details of the errors' effect on the accuracy of coupling measurement and how to overcome them can be found in original works [60, 61, 51]. In addition, a good $\mathrm{S} / \mathrm{N}$ ratio is critical for obtaining accurate couplings because the J-modulation method is based on intensity analysis. In frequency-displacement experiments, measurement accuracy is proportional to both the S/N ratio and spectral resolution, and is inversely related to peak linewidth [61]. For large macromolecules, differential relaxation can significantly broaden one of the two components, thus making accurate determination of peak position difficult. In addition, peak overlaps and imperfect pulse phase can also introduce errors in the measurement of peak positions.

As discussed above, a good $\mathrm{S} / \mathrm{N}$ ratio is critical to coupling measurement accuracy in both approaches. The $\mathrm{S} / \mathrm{N}$ ratio of oriented samples is usually lower than that of corresponding isotropic samples due to the lower concentration of oriented samples compared to isotropic samples [36], the broader linewidth caused by RDCs and a possible reduction in the tumbling rate of aligned biomolecules. The following techniques are generally used for increasing the $\mathrm{S} / \mathrm{N}$ ratio:

1. Sensitivity enhancement using multiple-quantum (MQ) coherence transfer for an isolated heteronuclear spin pair [62], TROSY technique [3] for large-sized proteins with or without perdeuteration, and RANCE-KAY scheme [63, 64].

2. Removal of the unwanted proton homo-coupling interactions ( $\mathrm{J}$ and RDC) either using shaped pulse in the indirect dimension [65, 66] or using band-selective adiabatic decoupling during the acquisition [67].

3. Removal of the unresolved couplings between ${ }^{15} \mathrm{~N}$ and remote protons in TROSY type experiments via use of the G-BIRD (gradient bilinear rotation decoupling) module in the $t_{1}$ dimension. The ${ }^{15} \mathrm{~N}$ linewidth of ubiquitin can be reduced by as much as $25 \%$ using this technique [68].

4. More recently, a numerically optimized coherence transfer scheme has been developed, which allows for selective observation of individual ${ }^{1} \mathrm{H}$-transitions in ${ }^{13} \mathrm{C}$-labeled methyl groups with optimal sensitivity. The applications of this technique to the measurement of ${ }^{1} \mathrm{H}-{ }^{1} \mathrm{H}$ and ${ }^{1} \mathrm{H}-{ }^{13} \mathrm{C}$ couplings in magnetically equivalent $\mathrm{CH}_{3}$ groups have been demonstrated [69, 70].

\section{Measurement of residual dipolar couplings in proteins}

RDC measurement is a prerequisite for the application of RDCs to studying the structure, dynamics and biochemical properties of biomolecules. The RDC measurement experiments published prior to 2001 have been recently reviewed [36]. In this review, we will concentrate on newly-developed pulse sequences whose applications have been demonstrated on both isotropic and anisotropic protein samples. We have tried our best to cover the literature as comprehensively as possible. However, since RDC measurement is still an active research area in biomolecular NMR and new results are being published daily, we may still have missed some important recent results. In the following sections, we will first describe experiments for measuring individual 


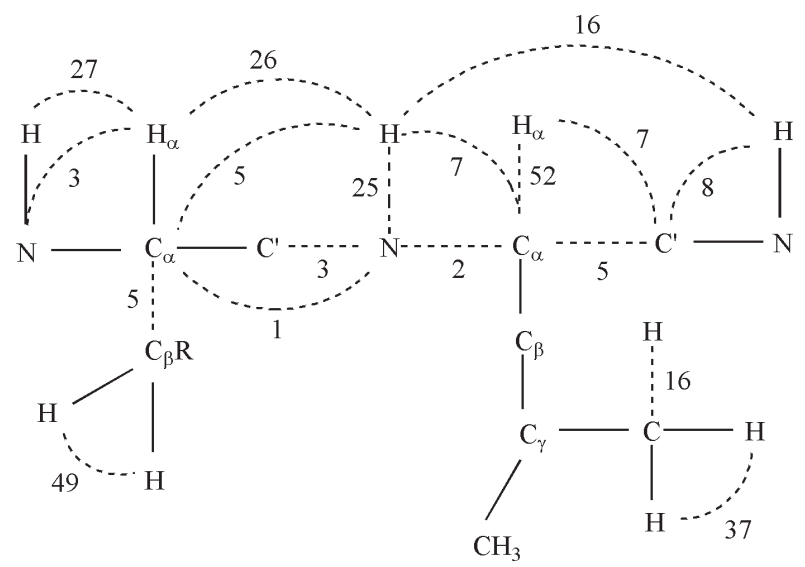

Figure 5: The calculated maximal RDCs (relative to $25 \mathrm{~Hz}$ of a ${ }^{1} \mathrm{H}-{ }^{15} \mathrm{~N}$ spin pair) on proteins. All these RDCs can be experimentally measured (see this review). Some of these numbers are taken directly from Permi and Annila's work [71] or calculated based on relative scales given in Table 1 in [36]. Bond lengths and angles used to calculate RDCs are taken from [72]. The bond lengths of $\mathrm{H}-\mathrm{N}$ and $\mathrm{C}-\mathrm{H}$ are $1.04 \AA ̊$ and $1.09 \AA$, respectively. For spin pairs separated by three or more bonds, only the shortest possible distance is used. The shortest distance for the intra ${ }^{1} \mathrm{H}_{\alpha}-\mathrm{H}_{\mathrm{N}}$ is $2.17 \AA$, taken from ubiquitin $\mathrm{X}$-ray structure [73]. The distances between $\mathrm{H}_{\mathrm{N}}(\mathrm{i})$ and $\mathrm{H}_{\alpha}(\mathrm{i}-1) / \mathrm{H}_{\mathrm{N}}(\mathrm{i}-1)$ are $2.2 \AA / 2.6 \AA$, respectively [74]. The value of ${ }^{1} \mathrm{H}^{-}{ }^{1} \mathrm{H}$ RDC $\left(\mathrm{D}_{\mathrm{HH}}\right)$ in a methyl group was calculated using $\mathrm{D}_{\mathrm{HH}}=2.3 \mathrm{D}_{\mathrm{CH}}$ [75], where the $\mathrm{D}_{\mathrm{CH}}$ is the $\mathrm{RDC}$ of $\mathrm{C}-\mathrm{H}$ vector in a methyl group.

types of RDC, then present experiments for simultaneous measurement of several types of RDCs. To facilitate the discussion, shown in Fig. 5 are the magnitudes of some measurable RDCs on proteins.

\section{1 $\quad{ }^{1} \mathbf{H}-{ }^{15} \mathbf{N}$ dipolar coupling}

Both NH $\left({ }^{1} \mathrm{H}^{-15} \mathrm{~N}\right)$ and $\mathrm{CH}\left({ }^{13} \mathrm{C}_{\alpha^{-}}{ }^{1} \mathrm{H}_{\alpha}\right)$ RDCs can be measured more accurately than other backbone onebond RDCs because their dipolar couplings are large (Fig. 5) due to the large ${ }^{1} \mathrm{H}$ gyromagnetic ratio and short bond lengths (Eq. (11)). These RDCs have been used most frequently in various biochemical applications. Compared to CH RDCs, NH RDCs can be measured more easily since the interference from other nuclei can be readily removed, and $\left[{ }^{1} \mathrm{H},{ }^{15} \mathrm{~N}\right]$ correlated spectra are generally well resolved. Previously, NH RDCs were usually measured using $\left[{ }^{1} \mathrm{H},{ }^{15} \mathrm{~N}\right]$-HSQC experiments with either J-resolved or J-quantitative modulation approaches $[50,51,58]$. To reduce the overlap of coupled spectra, an IPAP-HSQC experiment [38] was introduced. More recent developments have focused on enhancing resolution and improving the sensitivity, especially for large molecules.

The DSSE-HSQC (Doublet Separation and Sensitivity Enhancement) experiment [76] combines features of both IPAP [38] techniques and sensitivity enhancement schemes [64]. The sequence is similar to the TROSY experiment, with ${ }^{15} \mathrm{~N}$ decoupled during data acquisition. In each $t_{1}$-increment, four FIDs are recorded in order to perform simultaneous quadrature detection and spin-state-editing. The precision on ubiquitin is $0.05 \mathrm{~Hz}$. The precision on hepatitis $\mathrm{C}$ protease complex is estimated to be $0.13 \mathrm{~Hz}$; this protein has an isotropic rotational correlation time of $10.1 \mathrm{~ns}$ at $37^{\circ} \mathrm{C}$.

A suite of $2 \mathrm{D}\left[{ }^{1} \mathrm{H},{ }^{15} \mathrm{~N}\right]$ correlation experiments have been proposed for different size proteins by choosing different components of multiplets caused by a ${ }^{1} \mathrm{H}_{-}{ }^{15} \mathrm{~N}$ coupling [55]. Fig. 6 illustrates schematically how to use spin-state-selective coherence transfer $\left(\mathrm{S}^{3} \mathrm{CT}\right)$ and a modified TROSY sequence to determine ${ }^{1} \mathbf{J}_{\mathrm{NH}}$ by selectively observing only one out of four possible components. Shown in Fig. 6a is the overlay of two subspectra through a linear combination of two data sets from a modified TROSY sequence. The two peaks used 

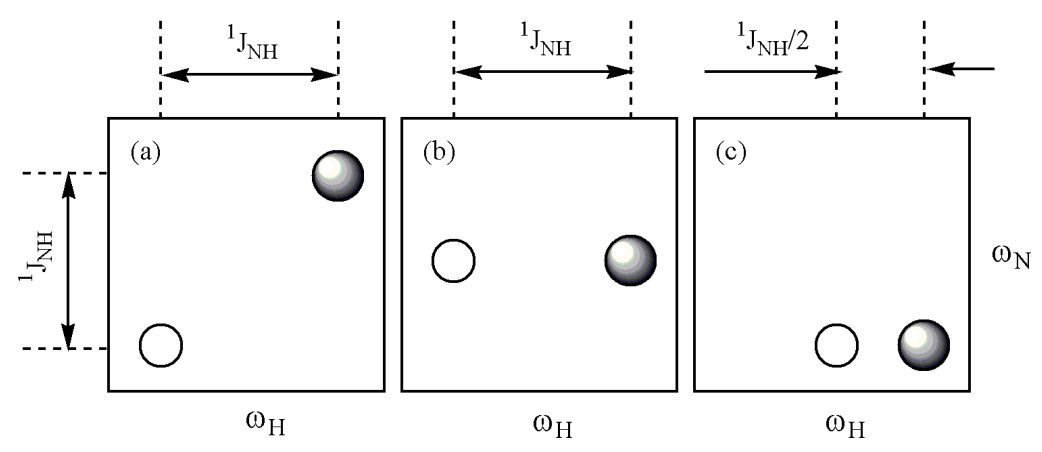

Figure 6: Overlays of two different 2D $\left[{ }^{15} \mathbf{N},{ }^{1} \mathbf{H}\right]$-correlated spectra (gray and blank circles). These different experiments are designed to measure couplings on different sized-protein samples. The overlays in a) and c) are from modified TROSY sequences; $b$ ) is from $\mathrm{S}^{3} \mathrm{CT}$ sequences.

to extract the couplings are called anti-TROSY/TROSY and TROSY/anti-TROSY (see Fig. 4), respectively. This approach is applicable to medium-sized molecules. Shown in Fig. $6 \mathrm{~b}$ is the overlay of two sub-spectra from the $\mathrm{S}^{3} \mathrm{CT}$ sequence, where ${ }^{15} \mathrm{~N}$ is decoupled from ${ }^{1} \mathrm{H}$. The doublet of an ${ }^{1} \mathrm{H}$ spin is separated into two different spectra depending on whether it is coupled to the $\alpha$ or $\beta$ spin state of the ${ }^{15} \mathrm{~N}$ nucleus. This approach is applicable to small-sized molecules because one component is neutral/anti-TROSY peak. For large-sized molecules, a combination of regular TROSY and ${ }^{15} \mathrm{~N}$ decoupled TROSY experiments can be used. The overlay of the spectra from these two experiments are shown in Fig. 6c. The methods have been demonstrated on ${ }^{15} \mathrm{~N}$-labeled protein chymotrypsin inhibitor 2 in a lipid bicelle mixture.

TROSY-based HNCO experiments can be used to measure five different dipolar couplings on deuterated large-sized proteins [77]. Here we will only discuss the strategy used to measure ${ }^{1} \mathrm{~J}_{\mathrm{NH}}$ and ${ }^{1} \mathrm{D}_{\mathrm{NH}}$; applications to the measurement of other RDCs will be discussed later. ${ }^{1} \mathbf{J}_{\mathrm{NH}}$ couplings are measured in two separate experiments with three resonances at $\left(\omega_{\mathrm{C}^{\prime}}-\pi^{2} \mathrm{~J}_{\mathrm{HC}^{\prime}}, \omega_{\mathrm{N}}-(\kappa+1) \pi^{1} \mathrm{~J}_{\mathrm{NH}}, \omega_{\mathrm{HN}}+\pi^{1} \mathrm{~J}_{\mathrm{NH}}\right)$, where $k$ is a J-enhancement factor with values of 0 and 2 . The chemical shifts for a given particular cross peak in two experiments are separated by ${ }^{1} \mathrm{~J}_{\mathrm{NH}}$ along the ${ }^{15} \mathrm{~N}$ dimension. Since both experiments only observe the narrowest component (the TROSY peak), TROSY-HNCO is a highly sensitive and resolution-optimized experiment for large-sized proteins with or without perdeuteration. It has been tested on an ${ }^{15} \mathrm{~N},{ }^{13} \mathrm{C},{ }^{2} \mathrm{H}$-labeled human carbonic anhydrase II (259 residues), and on a complex of Val, Leu, Ile ( $\delta_{1}$ only) methyl protonated ${ }^{15} \mathrm{~N} /{ }^{13} \mathrm{C} /{ }^{2} \mathrm{H}$-labeled maltose binding protein (MBP, 370 residues) and $\beta$-cyclodextrin. The average value for ${ }^{1} \mathrm{~J}_{\mathrm{NH}}$ measured in the unoriented $1 \mathrm{mM}$ MBP sample is $-93.7 \pm 1.1 \mathrm{~Hz}$.

$(\delta, \mathrm{J})$-E.COSY HSQC [78] is an experiment where the doublet in an E.COSY pattern is separated by $2 \omega_{N}$ along the ${ }^{15} \mathrm{~N}$ dimension. The experiment differs from the regular water gate-HSQC in two aspects. Firstly, there is an extra $90^{\circ}$ pulse on ${ }^{15} \mathrm{~N}$ before the acquisition recovers both orthogonal transverse magnetization components in a way that is similar to the sensitivity enhancement scheme [63], but there is no extra INEPT unit. Secondly, it leaves ${ }^{15} \mathrm{~N}$ coupling on during the acquisition period. The spectral width in the ${ }^{15} \mathrm{~N}$ dimension is set to twice the ${ }^{15} \mathrm{~N}$ chemical shift range. The final spectrum is composed of two distinct regular HSQC spectra with cross peak resonances at $\left(\omega_{\mathrm{N}}, \omega_{\mathrm{H}}+\pi \mathrm{J}_{\mathrm{NH}}\right)$ and $\left(-\omega_{\mathrm{N}}, \omega_{\mathrm{H}}-\pi \mathrm{J}_{\mathrm{NH}}\right)$, respectively. Thus the E.COSY pattern is composed of frequency displacement of $2 \omega_{N}$ along the ${ }^{15} \mathrm{~N}$ dimension and ${ }^{1} \mathbf{J}_{\mathrm{NH}}$ along the ${ }^{1} \mathrm{H}$ dimension. The accuracy of the coupling measurement is controlled by the digital resolution along the ${ }^{1} \mathrm{H}$ dimension. The pulse scheme has been demonstrated on a $1.0 \mathrm{mM}{ }^{15} \mathrm{~N},{ }^{13} \mathrm{C}$-labeled protein $\mathrm{GB} 1$ with 56 residues. One attractive feature of this approach is that there is no interference of unwanted components due to the $\mathbf{J}$ mismatch as seen in the spin-state-selective approaches such as the IPAP-HSQC or $\mathrm{S}^{3} \mathrm{E}$. Another 
advantage is convenience: it is easier to extract couplings from a single spectrum than from two sub-spectra.

Sensitivity-enhanced IPAP, TROSY-anti-TROSY and E.COSY HSQC experiments provide choices for measuring ${ }^{1} \mathrm{D}_{\mathrm{NH}}{ }^{2}$ on proteins of different sizes [79]. The principles for sensitivity improvement and the appearances of spectra acquired from the three experiments are similar to that of the $(\delta, \mathrm{J})$-E.COSY HSQC experiment discussed above. The key difference is that the couplings are encoded in the ${ }^{15} \mathrm{~N}$ dimension, rather than the ${ }^{1} \mathrm{H}$ dimension. All three experiments have been tested on GB1 protein (56 residues); authors [79] suggested that sensitivity-enhanced IPAP-HSQC can be used for large-sized protein samples. Interestingly, the authors found that the RDCs measured from the ${ }^{1} \mathrm{H}$ dimension are always smaller than those from the ${ }^{15} \mathrm{~N}$ dimension. Further analysis revealed that systematic deviations arise from the influence of un-resolved three-bond ${ }^{1} \mathrm{H}_{\mathrm{N}}-{ }^{1} \mathrm{H}_{\alpha}$ couplings and cross-correlated relaxation between ${ }^{1} \mathrm{H}^{-15} \mathrm{~N}$ and ${ }^{1} \mathrm{H}_{\mathrm{N}}-{ }^{1} \mathrm{H}_{\alpha}$ dipolar couplings.

Quantitative J-modulated HSQC [80] is an experiment similar to works previously published by Tjandra and coworkers [50] and Tolman and coworkers [51]. In Tjandra's approach, the peak intensity is not only modulated by couplings but also the $\mathrm{T}_{2}$ relaxation rate of ${ }^{15} \mathrm{~N}$ nuclei, while in Tolman and Prestegard's approach, the $\mathrm{J}$ coupling is extracted from the intensity ratios of both in-phase and anti-phase peaks from the same constant time period. The quantitative J modulated HSQC proposed by McFeetsers and coworkers uses constant time HSQC so that the peak intensity is not a function of the transverse relaxation of ${ }^{15} \mathrm{~N}$ nuclei. The $\mathrm{J}$-modulation is realized by moving the refocused $180^{\circ}{ }^{15} \mathrm{~N}$ pulse within the constant time period $\left(2 \mathrm{~T}_{N}=33.4\right.$ $\mathrm{ms}$ ) according to a series of positions $(\Delta)$. The coupling values are determined by fitting peak intensities to a function of $I_{0} \cos \left(2 \pi \mathrm{J}_{\mathrm{HN}}\left(\mathrm{T}_{\mathrm{N}}-\Delta\right)\right)$. The sequence has been tested on three proteins: ubiquitin in both isotropic and oriented condition, interleukin-4 (IL-4, $16 \mathrm{kDa})$ and NusB (18 kDa) in isotropic condition. The precision on ubiquitin is $0.07 \mathrm{~Hz}$ without data mirroring and $0.04 \mathrm{~Hz}$ with data mirroring. The precision obtained under the same experimental condition using IPAP-HSQC is $0.06 \mathrm{~Hz}$.

It is worth mentioning that an experiment has been developed to measure couplings from individual vectors of ${ }^{1} \mathrm{H}^{\mathrm{N} 1}{ }_{-}^{15} \mathrm{~N}$ and ${ }^{1} \mathrm{H}^{\mathrm{N} 2}{ }_{-}^{15} \mathrm{~N}$ in Asn and Gln side chains. It is an $\mathrm{NH}_{2}$-edited and spin-state-selected HSQC experiment. The couplings of individual vectors can be extracted from the frequency displacement along the ${ }^{1} \mathrm{H}$ dimension in two-subspectra [81].

\section{2 ${ }^{13} \mathbf{C}_{\alpha}{ }^{-1} \mathbf{H}_{\alpha}$ dipolar coupling}

The $\mathrm{CH}$ RDC of a ${ }^{13} \mathrm{C}_{\alpha^{-}}{ }^{1} \mathrm{H}_{\alpha}$ bond is about 2 times larger than the $\mathrm{NH}$ RDC of a ${ }^{1} \mathrm{H}_{-}{ }^{15} \mathrm{~N}$ bond (Fig. 5), and can be as large as $50 \mathrm{~Hz}$ under the conditions routinely used for anisotropic samples [36]. Even with low precision, $\mathrm{CH}$ RDCs are very useful in structure determination and refinement since the orientational information extracted from CH RDCs is non-redundant to that provided by NH RDCs [82]. Previously, CH RDCs were measured from constant-time $\left[{ }^{1} \mathrm{H},{ }^{13} \mathrm{C}\right]$ correlated HSQC experiments by a peak intensity modulation method [83]. The method is applicable to small-sized proteins, but not practical for medium- or large-sized proteins due to poor dispersion in ${ }^{1} \mathrm{H}_{\alpha} /{ }^{13} \mathrm{C}_{\alpha}$ regions. Two approaches have been developed to alleviate the overlap problem. The first is to make use of the good dispersion of $\left[{ }^{1} \mathrm{H},{ }^{15} \mathrm{~N}\right]$ correlation by encoding ${ }^{1} \mathrm{~J}_{\mathrm{C}_{\alpha} \mathrm{H}_{\alpha}}$ couplings into $\left[{ }^{1} \mathrm{H},{ }^{15} \mathrm{~N}\right]$ correlation. The second is to use an extra dimension to enhance the resolution.

The regular 3D (HA)CA(CO)NH experiment has been modified to measure ${ }^{1} \mathrm{~J}_{\mathrm{C}_{\alpha} \mathrm{H}_{\alpha}}$ and ${ }^{1} \mathrm{D}_{\mathrm{C}_{\alpha} \mathrm{H}_{\alpha}}$ by encoding CH RDCs into a ${ }^{13} \mathrm{C}$-dimension [36, 43]. The coherence is transferred as follows:

\footnotetext{
${ }^{2}$ As shown in Section 1.1, any experiment for measuring scalar couplings can also be used to measure the corresponding RDCs. Hence, we use the following convention for notations: if the scalar coupling is denoted be $\mathrm{J}$, then the corresponding RDC will be denoted by D. For example, ${ }^{1} \mathrm{~J}_{\mathrm{NH}}$ is used to represent the one-bond scalar coupling between an amide nitrogen and the attached amide proton, the corresponding NH RDC is represented by ${ }^{1} \mathrm{D}_{\mathrm{NH}}$.
} 


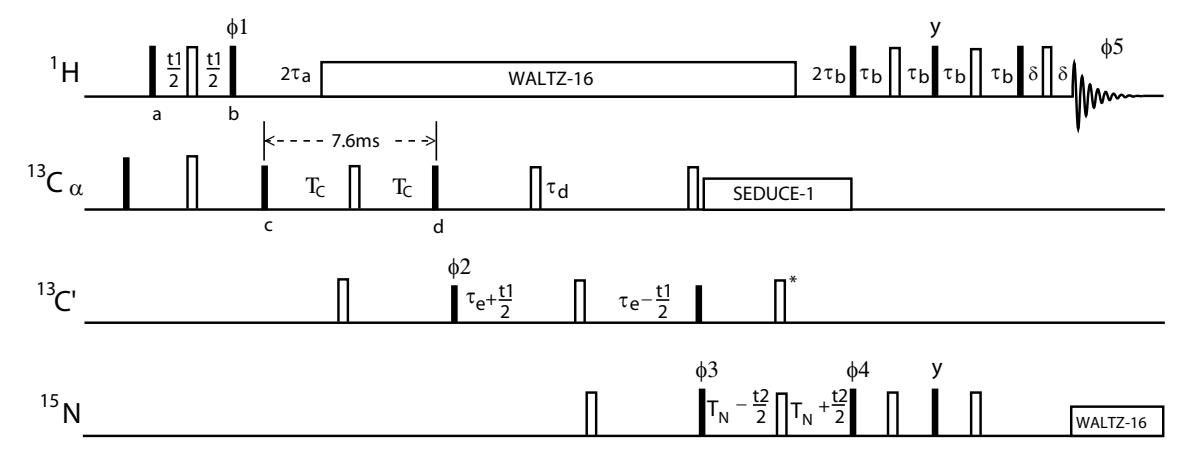

PFG

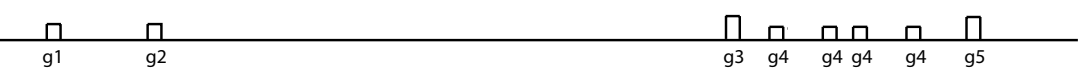

Figure 7: The pulse sequence HA(CA)CONH. Narrow filled and wide blank bars stand for $90^{\circ}$ and $180^{\circ}$ pulses. Phases of pulses are on $x$-axis unless indicated otherwise. Please note that the $180^{\circ}$ pulse on ${ }^{13} \mathrm{C}^{\prime}$ marked with " ** was mistakenly missed in the original paper. Technical details can be found in the original work. The figure was adapted from [44], with permission from Elsevier.

$$
{ }^{1} \mathrm{H}_{\alpha}(\mathrm{i}-1) \rightarrow{ }^{13} \mathrm{C}_{\alpha}(\mathrm{i}-1)\left(\mathrm{CT}, \mathrm{t}_{1}\right) \rightarrow{ }^{13} \mathrm{C}^{\prime}(\mathrm{i}-1) \rightarrow{ }^{15} \mathrm{~N}(\mathrm{i})\left(\mathrm{t}_{2}\right) \rightarrow{ }^{1} \mathrm{H}_{\mathrm{N}}(\mathrm{i})\left(\mathrm{t}_{3}\right)
$$

A constant time, $\mathrm{CT}=1 / \mathrm{J}_{\mathrm{C}_{\alpha} \mathrm{C}_{\beta}} \approx 28 \mathrm{~ms}$, is used in the ${ }^{13} \mathrm{C}$ evolution dimension to suppress the coupling interaction between $\mathrm{C}_{\alpha}$ and $\mathrm{C}_{\beta}$. The doublets of ${ }^{13} \mathrm{C}_{\alpha}-\left\{{ }^{1} \mathrm{H}_{\alpha}\right\}$ are in anti-phase mode because the $\sin \pi^{1} \mathrm{~J}_{\mathrm{C}_{\alpha} \mathrm{H}_{\alpha}}$ term is detected. Unfortunately, the long-delay $\left(1 / \mathrm{J}_{\mathrm{C}_{\alpha} \mathrm{C}_{\beta}}\right)$ reduces the sensitivity significantly for medium or large-sized proteins due to fast $\mathrm{T}_{2}$ relaxation of $\mathrm{C}_{\alpha}$. Another concern is that the cross correlation of $\mathrm{C}_{\alpha}-\mathrm{CSA}$ and ${ }^{13} \mathrm{C}_{\alpha^{-}}{ }^{1} \mathrm{H}_{\alpha}$ DD interaction is not canceled during the $t_{1}$ period, leading to different intensities for the two components of a doublet, even though the TROSY effect of a ${ }^{13} \mathrm{C}_{\alpha^{-}}{ }^{1} \mathrm{H}_{\alpha}$ spin pair is rather small [3].

A 3D $\mathrm{HNCO}(\mathrm{CA})$ experiment has been proposed [84] to measure $\mathrm{CH}$ RDCs by modifying the out-andback HN(CO)CA [85]. Firstly, a spin-state-filter $\left(\alpha / \beta-{ }^{1} \mathrm{~J}_{\mathrm{C}_{\alpha} \mathrm{H}_{\alpha}}\right)$, in a fashion of IPAP [38], is inserted right after the magnetization has been transferred from $\mathrm{C}^{\prime}$ to $\mathrm{C}_{\alpha}$. Post-acquisition addition and subtraction of the IP and AP spectra separates ${ }^{13} \mathrm{C}_{\alpha}-\left\{{ }^{1} \mathrm{H}_{\alpha}\right\}$ doublets into two sub-spectra. Secondly, the ${ }^{13} \mathrm{C}_{\alpha}-\left\{{ }^{1} \mathrm{H}_{\alpha}\right\}$ doublet are concatenated with $\mathrm{C}^{\prime}$ chemical shift evolution to take advantage of the better chemical shift dispersion in $\mathrm{C}^{\prime}$. The experiment has been tested on $1.0 \mathrm{mM}{ }^{13} \mathrm{C} /{ }^{15} \mathrm{~N}$-labeled ubiquitin. The pair-wise RMSD is $1.35 \mathrm{~Hz}$ for 63 residues, according to two independent measurements. The drawback of this approach is that the coupling of $\mathrm{C}_{\alpha}-\mathrm{C}_{\beta}$ is also active during the ${ }^{13} \mathrm{C}_{\alpha^{-}}{ }^{1} \mathrm{H}_{\alpha}$ coupling evolution period, limiting the maximum acquisition time for ${ }^{13} \mathrm{C}_{\alpha^{-}}{ }^{1} \mathrm{H}_{\alpha}$ coupling evolution, and may potentially reduce measurement precision due to line broadening from $\mathrm{C}_{\alpha}-\mathrm{C}_{\beta}$ couplings.

2D (HACACO)NH is a quantitative ${ }^{1} \mathrm{~J}_{\mathrm{C}_{\alpha} \mathrm{H}_{\alpha}}$-modulated triple resonance experiment [86] with a coherence transfer pathway similar to that of $(\mathrm{HA}) \mathrm{CA}(\mathrm{CO}) \mathrm{NH}[36,43]$. After the magnetization of ${ }^{1} \mathrm{H}_{\alpha}$ is converted into $\mathrm{C}_{\alpha}^{y} \mathrm{H}_{\alpha}^{z}$, the antiphase term is refocused by a variable delay over a constant time period (CT = $\left.28 \mathrm{~ms}\right)$. Signal magnitudes are thus modulated by $\sin \left(\pi^{1} \mathrm{~J}_{\mathrm{C}_{\alpha} \mathrm{H}_{\alpha}} \Delta\right)$. The magnetization is finally transferred to ${ }^{15} \mathrm{~N}$ through $\mathrm{C}^{\prime}$, and a $2 \mathrm{D}\left[{ }^{1} \mathrm{H},{ }^{15} \mathrm{~N}\right]$-HSQC spectrum is acquired. Thus, the resolution is much higher than that in $\left[{ }^{1} \mathrm{H}\right.$, ${ }^{13} \mathrm{C}$ ]-HSQC experiments [83]. Another advantage of this approach is that no ${ }^{1} \mathrm{H}_{\alpha} /{ }^{13} \mathrm{C}_{\alpha}$ assignment is needed in order to obtain CH RDCs. The experiment has been demonstrated on a 15-kDa RNA binding domain of E. coli Rho factor. The average error in the measured coupling constants was less than $0.8 \%$. The authors 
anticipated that the experiment should be applicable to fully protonated medium-sized proteins $(20 \sim 25 \mathrm{kDa})$, although the $\mathrm{C}_{\alpha}$ transverse relaxation is active for about $28 \mathrm{~ms}$ during the constant time period. Later on, a modified version was published [80] where the duration of constant time is shortened in order to limit the sensitivity loss due to fast $\mathrm{T}_{2}$ relaxation of $\mathrm{C}_{\alpha}$.

3D HA(CA)CONH experiment uses the same coherence transfer pathway as in $(\mathrm{HA}) \mathrm{CA}(\mathrm{CO}) \mathrm{NH}$ [43], with critical improvement in sensitivity [44]. The key features of $\mathrm{HA}(\mathrm{CA}) \mathrm{CONH}$ are as follows:

1. In order to avoid a long $\mathrm{T}_{2}$ relaxation of $\mathrm{C}_{\alpha}$ during constant-time period, the ${ }^{1} \mathrm{~J}_{\mathrm{C}_{\alpha}} \mathrm{H}_{\alpha}$ coupling evolution is moved to the ${ }^{1} \mathrm{H}_{\alpha}$ dimension at the beginning of the sequence (point a to $\mathrm{b}$ as shown in Fig. 7), and combined with the magnetization transfer from ${ }^{1} \mathrm{H}_{\alpha}$ to ${ }^{13} \mathrm{C}_{\alpha}$.

2. Simultaneous $180^{\circ}$ pulses are applied to both ${ }^{1} \mathrm{H}$ and ${ }^{13} \mathrm{C}$ to remove effects of CSA and DD crosscorrelation resulting more uniform peak intensities for anti-phase doublets.

3. From the point $\mathrm{c}$ to $\mathrm{d}$, the magnetization is further relayed to $\mathrm{C}^{\prime}$ and the duration is set to $7.6 \mathrm{~ms}$, which is an optimized value to suppress coherence dephase due to $\mathrm{C}_{\alpha}-\mathrm{C}_{\beta}$ couplings, and to maintain the transfer efficiency from $\mathrm{C}_{\alpha}$ to $\mathrm{C}^{\prime}$. The duration of the same period is set to $28 \mathrm{~ms}$ as in the (HA)CA(CO)NH sequence.

4. The ${ }^{1} \mathrm{~J}_{\mathrm{C}_{\alpha} \mathrm{H}_{\alpha}}$ coupling evolution is concatenated with the $\mathrm{C}^{\prime}$ chemical shift labeling period to take advantage of the favorable resonance dispersion of $\mathrm{C}^{\prime}$ nucleus.

Since the constant time duration is reduced from $28 \mathrm{~ms}$ to $7.6 \mathrm{~ms}$, the intensity loss due to $\mathrm{T}_{2}$ relaxation of $\mathrm{C}_{\alpha}$ is reduced significantly. The sequence has been tested on a C-terminal domain of the human Ku- 80 protein (152 residues) at a concentration of $1.2 / 0.6 \mathrm{mM}$ for isotropic/anisotropic samples. The results showed that sensitivity enhancement is profound for structured regions, with an average of 50-60\% enhancement being observed when compared with the $(\mathrm{HA}) \mathrm{CA}(\mathrm{CO}) \mathrm{NH}$ sequence. It should be applicable to a protein with a molecular weight of $20 \mathrm{kDa}$.

HCACO $\left(\alpha / \beta-\mathrm{J}_{-}{ }^{1} \mathrm{H}_{\alpha}-\mathrm{C}_{\alpha}\right)$ is an experiment designed to extract ${ }^{1} \mathrm{~J}_{\mathrm{C}_{\alpha} \mathrm{H}_{\alpha}}$ from samples not suitable for ${ }^{1} \mathrm{H}-$ ${ }^{15} \mathrm{~N}$ correlation detection [87]. These include samples that may require high $\mathrm{pH}$ or temperature, where the amide protons exchange so fast with water that the sensitivity of $\left[{ }^{1} \mathrm{H},{ }^{15} \mathrm{~N}\right]$ correlation experiment is greatly reduced, and the proline-rich proteins that lack many amide protons. This experiment is an out-and-back experiment and can be carried out in $\mathrm{D}_{2} \mathrm{O}$. The coherence is transferred as follows:

$$
{ }^{1} \mathrm{H}_{\alpha} \rightarrow{ }^{13} \mathrm{C}_{\alpha} \rightarrow{ }^{13} \mathrm{C}^{\prime}\left(\mathrm{t}_{1}\right) \rightarrow{ }^{13} \mathrm{C}_{\alpha}\left(\mathrm{CT}-\mathrm{t}_{2}\right) \rightarrow{ }^{1} \mathrm{H}_{\alpha}\left(\mathrm{t}_{3}\right)
$$

A spin-state-filter, $\mathrm{S}^{3} 90^{\circ}$ pulse on $\mathrm{C}_{\alpha}$ before the acquisition period was used to separate ${ }^{1} \mathrm{H}_{\alpha}-\left\{{ }^{13} \mathrm{C}_{\alpha}\right\}$ doublets into two sub-spectra [88]. The frequency displacement along the ${ }^{1} \mathrm{H}_{\alpha}$ dimension from the two spectra is the ${ }^{1} \mathrm{H}-{ }^{13} \mathrm{C}$ couplings. Although the experiment uses an approach similar to an earlier version of HCACO$\alpha / \beta$ [45], the sensitivity is improved by a factor of $\sqrt{2}$ because both orthogonal magnetization components are recovered after the $\mathrm{C}_{\alpha}$ evolution period. The pulse scheme has been demonstrated on a $1.5 \mathrm{mM}$ ubiquitin sample with a precision of a fraction of Hertz. Like all other spin-state-editing techniques, the $\mathrm{J}$ crosstalk due to $\mathrm{J}$ mismatch could affect the accuracy of measurement. The effect may not be significant since ${ }^{1} \mathrm{~J}_{\mathrm{C}_{\alpha} \mathrm{H}_{\alpha}}$ doublets are usually well resolved.

\section{3 $\mathrm{C}_{\alpha}-\mathrm{C}^{\prime}$ dipolar coupling}

The intrinsic RDC values of a $\mathrm{C}_{\alpha}-\mathrm{C}^{\prime}$ bond are about one-fifth of that of NH RDCs, but larger than both $\mathrm{C}^{\prime}-\mathrm{N}$ and $\mathrm{N}-\mathrm{C}_{\alpha}$ RDCs (Fig. 5). $\mathrm{C}_{\alpha}-\mathrm{C}^{\prime}$ RDCs can be measured easily with experiments such as 3D HNCO experiments without ${ }^{13} \mathrm{C}$ decoupling during the $\mathrm{C}^{\prime}$ chemical shift evolution [36, 89]. Since HNCO is the most sensitive and well-resolved triple resonance experiment, the approach is applicable for most medium-sized 
proteins. More recently, methods have been developed to extract the $\mathrm{C}_{\alpha}-\mathrm{C}^{\prime}$ couplings from either $2 \mathrm{D}{ }^{1} \mathrm{H}$ ${ }^{15} \mathrm{~N}$ HSQC experiments to save spectrometer time, or TROSY approaches for large-sized molecules. Pulse sequences have also been developed for specifically-labeled proteins, such as $\left[{ }^{13} \mathrm{C},{ }^{13} \mathrm{C}^{\prime},{ }^{15} \mathrm{~N}\right.$, $\left.{ }^{2} \mathrm{H}\right]$, where the interference from $\mathrm{C}_{\beta}$ can be removed. The $\mathrm{C}_{\alpha}-\mathrm{C}^{\prime}$ couplings can be directly observed from either HNCA or $\mathrm{HNCO}$ experiments with high precision on these labeled proteins. Alternatively, they can also be determined from J-modulated HNCA experiments where a constant time period is used in the $\mathrm{C}_{\alpha}$ chemical evolution pe$\operatorname{riod}[90]$.

$\mathrm{HN}(\alpha / \beta$-COCA-J $)$ is a modified $2 \mathrm{D}$ version of $\mathrm{HNCO}$ [91] for measuring $\mathrm{C}_{\alpha}-\mathrm{C}^{\prime}$ RDCs. The flow of coherence is:

$$
{ }^{1} \mathrm{H}_{\mathrm{N}} \rightarrow{ }^{15} \mathrm{~N} \rightarrow{ }^{13} \mathrm{C}^{\prime} \rightarrow{ }^{15} \mathrm{~N}\left(\mathrm{t}_{1}\right) \rightarrow{ }^{1} \mathrm{H}_{\mathrm{N}}\left(\mathrm{t}_{2}\right)
$$

The active $\mathrm{C}_{\alpha}-\mathrm{C}^{\prime}$ coupling and ${ }^{15} \mathrm{~N}$ evolution period $\left(t_{1}\right)$ are concatenated. To simplify the spectra, a spinstate-filter element (using $\mathrm{J}_{\mathrm{C}_{\alpha} \mathrm{C}^{\prime}}$ ) similar to IPAP [38] is used prior to $t_{1}$ evolution to enhance the resolution. The doublet are separated into two-subspectra. The spin-state-filtration is very efficient. Assuming a deviation of up to $\pm 10 \mathrm{~Hz}$ from the tuned $55 \mathrm{~Hz}$ of a $\mathrm{C}_{\alpha}-\mathrm{C}^{\prime}$ coupling, the intensity of the minor component is 30 times smaller than that of the major peak. The pulse sequence was tested on $1.0 \mathrm{mM}$ regulatory domain of human cardiac tropnin (cTnC, $10 \mathrm{kDa})$. Similarly, a 2D $\mathrm{HN}\left(\mathrm{CO}-\alpha / \beta-\mathrm{C}^{\prime} \mathrm{C}_{\alpha}-\mathrm{J}\right)$-TROSY [71] experiment, modified from a TROSY-HNCO experiment [92], has been proposed for large-sized proteins and can be easily extended to $3 \mathrm{D}$. The sequence has been tested on $18 \mathrm{kDa} \mathrm{U}-\left({ }^{15} \mathrm{~N},{ }^{13} \mathrm{C}\right) \mathrm{cNTnC}$ protein in both a dilute liquid crystal medium $(0.5 \mathrm{mM})$ and isotropic solution $(2.0 \mathrm{mM})$.

2D HN(J-COCA) is a quantitative J-modulated HNCO experiment [80]. The coherence is transferred as follows:

$$
{ }^{1} \mathrm{H}_{\mathrm{N}} \rightarrow{ }^{15} \mathrm{~N} \rightarrow{ }^{13} \mathrm{C}^{\prime} \cos \left(2 \pi \mathrm{J}_{\mathrm{C} \alpha \mathrm{C}^{\prime}} \Delta\right) \rightarrow{ }^{15} \mathrm{~N}\left(\mathrm{t}_{1}\right) \rightarrow{ }^{1} \mathrm{H}_{\mathrm{N}}\left(\mathrm{t}_{2}\right)
$$

After the magnetization transfer to ${ }^{13} \mathrm{C}^{\prime}$, a constant time period $(\approx 56 \mathrm{~ms})$ is used, during which a series of delays allow the evolution of $\mathrm{C}_{\alpha}-\mathrm{C}^{\prime}$ couplings, which can then be extracted from a curve fitting of peak intensities versus the coupling evolution time. The average error is less than $\pm 0.05 \mathrm{~Hz}$ on ubiquitin. For large-sized molecules, the sensitivity can be potentially improved by adapting TROSY schemes. Another Jmodulated 2D HN(J-CACO) experiment to measure the couplings of $\mathrm{C}_{\alpha}-\mathrm{C}^{\prime}$ is also proposed in the same paper. The experiment starts from ${ }^{1} \mathrm{H}_{\alpha}$ and ends at $\mathrm{H}_{\mathrm{N}}$ with a coherence transfer similar to that in an (HA)CACONH experiment. The constant time for the $\mathrm{C}_{\alpha}-\mathrm{C}^{\prime}$ evolution is $28 \mathrm{~ms}$, during which the $\mathrm{C}_{\alpha}$ magnetization sits on the $\mathrm{X}-\mathrm{Y}$ plane. Since the $\mathrm{T}_{2}$ relaxation of $\mathrm{C}_{\alpha}$ is faster than $\mathrm{C}^{\prime}$, and the choice of constant time duration is restrained by $\mathrm{J}_{\mathrm{C}_{\alpha} \mathrm{C}_{\beta}}$, neither the intensity nor the precision of this scheme is as good as those of out-and-back experiments such as the $\mathrm{HN}(\mathrm{J}-\mathrm{COCA})$ experiment.

3D $\operatorname{HCACO}\left(\alpha / \beta-\mathrm{J}-\mathrm{C}^{\prime} \mathrm{C}_{\alpha}\right)$ is an out-and-back experiment with the following coherence transfer pathway [87]:

$$
{ }^{1} \mathrm{H}_{\alpha} \rightarrow{ }^{13} \mathrm{C}_{\alpha} \rightarrow{ }^{13} \mathrm{C}^{\prime}\left(\mathrm{t}_{1}\right) \rightarrow{ }^{13} \mathrm{C}_{\alpha}\left(\mathrm{CT}, \mathrm{t}_{2}\right) \rightarrow{ }^{1} \mathrm{H}_{\alpha}\left(\mathrm{t}_{3}\right)
$$

The evolution of $\mathrm{C}_{\alpha}-\mathrm{C}^{\prime}$ couplings is embedded in the $\mathrm{C}^{\prime}$ chemical shift evolution period. To simplify the spectra, a spin-state-filter (using large ${ }^{1} \mathbf{J}_{\mathrm{C}_{\alpha} \mathrm{H}_{\alpha}}$ ) is inserted prior to the $t_{1}$ period. Addition and subtraction of IPAP spectra result in two sub-spectra with cross peaks at $\left\{\omega_{\mathrm{C}^{\prime}}+\pi{ }^{1} \mathrm{~J}_{\mathrm{C}^{\prime} \mathrm{C} \alpha}, \omega_{\mathrm{C} \alpha}, \omega_{\mathrm{H} \alpha}\right\}$ and $\left\{\omega_{\mathrm{C}^{\prime}}-\right.$ $\left.\pi^{1} \mathrm{~J}_{\mathrm{C}^{\prime} \mathrm{C} \alpha}, \omega_{\mathrm{C} \alpha}, \omega_{\mathrm{H} \alpha}\right\}$, respectively. The couplings are extracted by a frequency-displacement method. The 
sequence has been demonstrated on human ubiquitin. The experiment is a useful alternative if the $\left[{ }^{1} \mathrm{H},{ }^{15} \mathrm{~N}\right]$ correlation experiment is not sensitive due to unfavorable $\mathrm{pH}$ or temperature conditions.

\section{4 $\mathbf{C}_{\alpha}-\mathbf{C}_{\beta}$ dipolar coupling}

Similar to ${ }^{1} \mathrm{~J}_{\mathrm{C}_{\alpha} \mathrm{H}_{\alpha}}$, the RDCs of $\mathrm{C}_{\alpha}-\mathrm{C}_{\beta}$ bonds provide non-redundant orientational constraints with respect to the RDCs of bond vectors located in a peptide plane. This information can be critical for the accurate determination of alignment tensors [93, 94]. In addition, $\mathrm{C}_{\alpha}-\mathrm{C}_{\beta}$ RDCs provide information on side chain orientation and backbone dihedral angles [95, 96]. Unfortunately, poor separation of the chemical shift ranges between $\mathrm{C}_{\alpha}$ and $\mathrm{C}_{\beta}$ groups, fast $\mathrm{T}_{2}$ relaxation of $\mathrm{C}_{\alpha}$, and medium-sized $\mathrm{J}_{\mathrm{C}_{\alpha} \mathrm{C}_{\beta}}$ couplings make the measurement of $\mathrm{C}_{\alpha}-\mathrm{C}_{\beta}$ RDCs a big challenge. There are not many experiments published to date for the measurement of $\mathrm{C}_{\alpha}-\mathrm{C}_{\beta}$ RDCs.

$\mathrm{HNCO}\left(\mathrm{C}_{\alpha}-\mathrm{C}_{\beta}\right.$-J)-TROSY is a modification of HNCO-TROSY [92], and the $\mathrm{C}_{\alpha}-\mathrm{C}_{\beta}$ couplings are embedded in the $\mathrm{C}^{\prime}$ chemical shift evolution period [97]. The coherence is transferred as follows:

$$
{ }^{1} \mathrm{H}_{\mathrm{N}} \rightarrow{ }^{15} \mathrm{~N} \rightarrow{ }^{13} \mathrm{C}^{\prime} \rightarrow \mathrm{C}_{\alpha} \cos \left(\pi \mathrm{J}_{\mathrm{C} \alpha \mathrm{C} \beta} \lambda \mathrm{t}_{1}\right) \rightarrow \mathrm{C}^{\prime}\left(\mathrm{t}_{1}\right) \rightarrow{ }^{15} \mathrm{~N}\left(\mathrm{t}_{2}\right) \rightarrow{ }^{1} \mathrm{H}_{\mathrm{N}}\left(\mathrm{t}_{3}\right)
$$

Both the $\mathrm{C}_{\alpha}-\mathrm{C}_{\beta}$ coupling and $\mathrm{C}^{\prime}$ chemical evolution are concatenated in the $t_{1}$ period, while the $\mathrm{C}^{\prime}$ chemical shift evolution is modulated by $\cos \left(\pi^{1} \mathrm{~J}_{\mathrm{C} \alpha \mathrm{C} \beta} \lambda t_{1}\right)$, where $\lambda$ is used to amplify the couplings in order to resolve the two components of the doublet. The split along the $\mathrm{C}^{\prime}$ dimension yields the $\mathrm{C}_{\alpha}-\mathrm{C}_{\beta}$ couplings. The sequence has been demonstrated on a ubiquitin sample with $\lambda$ set to 2 .

2D HN(J-CACB) is a quantitative J-modulated experiment which monitors the intensity of $\left[{ }^{1} \mathrm{H},{ }^{15} \mathrm{~N}\right]$ correlated peaks versus the evolution time of $\mathrm{J}_{\mathrm{C}_{\alpha} \mathrm{C}_{\beta}}$ [80]. The magnetization transfer scheme is as follows:

$$
{ }^{1} \mathrm{H}_{\alpha} \rightarrow{ }^{13} \mathrm{C}_{\alpha} \cos \left(2 \pi \mathrm{J}_{\mathrm{C} \alpha \mathrm{C} \beta}(\mathrm{CT}-\Delta)\right) \rightarrow \mathrm{C}^{\prime} \rightarrow{ }^{15} \mathrm{~N}\left(\mathrm{t}_{1}\right) \rightarrow{ }^{1} \mathrm{H}_{\mathrm{N}}\left(\mathrm{t}_{2}\right)
$$

A key feature of the experiment is that the signal intensity is modulated by $\mathrm{J}_{\mathrm{C}_{\alpha} \mathrm{C}_{\beta}}$ during a constant time period $(2 \mathrm{CT}=28.4 \mathrm{~ms})$, achieved by a very selective inversion pulse on $\mathrm{C}_{\beta}$ at different time intervals. The total coupling evolution time is $2(\mathrm{CT}-\Delta)$. Special attention should be given to the selective $180^{\circ}$ inversion pulse on $\mathrm{C}_{\beta}$, since the imperfection of this pulse can generate significant errors in the measured couplings. The experiment is not suitable for serine and threonine residues since their $\mathrm{C}_{\beta}$ chemical shifts are not well separated from $\mathrm{C}_{\alpha}$ chemical shifts. The total duration of the constant time is $\frac{1}{J_{\mathrm{C} \alpha \mathrm{C} \beta}} \approx 28 \mathrm{~ms}$, and thus the precision of the $\mathrm{J}$ measurement is compromised by the limited array points, although the precision can be improved by data-mirroring. When tested on ubiquitin, $81 \%$ of the possible $\mathrm{C}_{\alpha}-\mathrm{C}_{\beta}$ RDCs can be extracted.

\section{5 $\mathrm{N}-\mathrm{C}^{\prime}$ and $\mathrm{H}_{\mathrm{N}}-\mathrm{C}^{\prime}$ dipolar coupling}

The intrinsic RDC values between one-bond $\mathrm{N}-\mathrm{C}^{\prime}$ and two-bond $\mathrm{H}_{\mathrm{N}}-\mathrm{C}^{\prime}$ nuclei are, respectively, about 8 and 3 times smaller than that of ${ }^{1} \mathrm{D}_{\mathrm{NH}}$ (See Fig. 5). In principle, N-C' couplings can be measured from regular $\left[{ }^{1} \mathrm{H}\right.$, ${ }^{15} \mathrm{~N}$ ]-HSQC or TROSY experiments without the decoupling of $\mathrm{C}^{\prime}$ during an ${ }^{15} \mathrm{~N}$ evolution time. Furthermore, if the spin states of $\mathrm{C}^{\prime}$ are not perturbed after the ${ }^{15} \mathrm{~N}$ evolution and no decoupling is applied on $\mathrm{C}^{\prime}$ during the ${ }^{1} \mathrm{H}$ acquisition period, an E.COSY pattern appears in the $\left[{ }^{1} \mathrm{H},{ }^{15} \mathrm{~N}\right]$ correlation spectrum. Thus, both $\mathrm{N}-\mathrm{C}^{\prime}$ and $\mathrm{H}_{\mathrm{N}}-\mathrm{C}^{\prime}$ couplings can be measured from a single $\left[{ }^{1} \mathrm{H},{ }^{15} \mathrm{~N}\right]$ correlation experiment [36], as schematically illustrated in Fig. 3. N-C' splittings can also be measured along the ${ }^{15} \mathrm{~N}$ dimension in 3D HNCO-TROSY by omitting the ${ }^{13} \mathrm{C}^{\prime} 180^{\circ}$ pulse during the ${ }^{15} \mathrm{~N}$ evolution period [61]. In practice, the spectrum is crowded since there is twice the number of cross peaks compared to a regular HSQC experiment. It is quite difficult to obtain high precision data from the direct measurement of peak splitting since the $\mathrm{N}-\mathrm{C}^{\prime}$ scalar couplings are rather 
small. More sophisticated experiments have been designed to enhance both the resolution and precision.

2D IPAP-H(N)CO is designed to measure $\mathrm{H}_{\mathrm{N}}-\mathrm{C}^{\prime}$ couplings in a 2D $\mathrm{H}_{\mathrm{N}}-\mathrm{C}^{\prime}$ correlated experiment [38]. The coherence transfer is similar to that in $\mathrm{HNCO}$ except that $\mathrm{C}^{\prime}$ and $\mathrm{H}_{\mathrm{N}}$ are detected. To resolve the doublets, a spin-state-filter (use $\mathrm{J}_{\mathrm{HN}}$ ) is inserted in the INEPT step for magnetization transfer from $\mathrm{C}^{\prime}$ to ${ }^{15} \mathrm{~N}$. Thus, two interleaved AP/IP experiments detect, respectively, the $2 \mathrm{~N}_{\mathrm{z}} \mathrm{C}_{\mathrm{y}}^{\prime}$ and $4 \mathrm{H}_{\mathrm{z}} \mathrm{N}_{\mathrm{z}} \mathrm{C}_{\mathrm{y}}^{\prime}$ terms. Through addition and subtraction of IP and AP spectra, doublets of $\mathrm{C}^{\prime}-\left\{{ }^{1} \mathrm{H}_{\mathrm{N}}\right\}$ are separated into two sub-spectra. The frequency displacement of the corresponding cross peaks in two sub-spectra along the $\mathrm{C}^{\prime}$ dimension yields the $\mathrm{C}^{\prime}-\mathrm{H}_{\mathrm{N}}$ couplings, although the values are small. The experiment has been demonstrated on a $0.7 \mathrm{mM}$ ubiquitin sample. When compared with the $\mathrm{C}^{\prime}$ coupled-E.COSY- $\left[{ }^{1} \mathrm{H},{ }^{15} \mathrm{~N}\right]$-HSQC experiment, the pairwise RMSD is 0.38 $\mathrm{Hz}$ for ${ }^{2} \mathrm{D}_{\mathrm{HN}-\mathrm{C}^{\prime}}$ with an error of less than $0.3 \mathrm{~Hz}$ in individual measurements. A very similar scheme called $\mathrm{H}\left(\alpha / \beta-\mathrm{NC}^{\prime}-\mathrm{J}\right) \mathrm{CO}$ experiment has also been proposed where the spin-state-filter part is inserted after the INEPT magnetization transfer step from $\mathrm{N}$ to $\mathrm{C}^{\prime}$ and prior to the $t_{1}$ period [98].

3D $\mathrm{HNCO}(\alpha / \beta$-NC'-J $)$ and 3D $\mathrm{HNCO}(\alpha / \beta$-NC'-J)-TROSY [97], based respectively on HNCO and HNCOTROSY schemes with the insertion of a spin-state-filter to increase the resolution, have been developed to measure $\mathrm{N}-\mathrm{C}^{\prime}$ couplings. $\mathrm{HNCO}\left(\alpha / \beta-\mathrm{NC}^{\prime}-\mathrm{J}\right)$ is suitable for small and medium-sized proteins, while $\mathrm{HNCO}(\alpha / \beta$ $\mathrm{NC}^{\prime}$-J)-TROSY has been tailored for medium and large-sized proteins. The magnetization transfer pathways of both experiments are very similar and can be described as follows:

$$
{ }^{1} \mathrm{H}_{\mathrm{N}} \rightarrow{ }^{15} \mathrm{~N} \rightarrow \mathrm{C}^{\prime}\left(\mathrm{t}_{1}\right) \rightarrow{ }^{15} \mathrm{~N}\left(\mathrm{t}_{2}\right) \rightarrow{ }^{1} \mathrm{H}_{\mathrm{N}}\left(\mathrm{t}_{3}\right)
$$

After the magnetization is transferred to $\mathrm{C}^{\prime}$ from ${ }^{15} \mathrm{~N}$, a spin-state-filter using large ${ }^{1} \mathbf{J}_{\mathrm{NH}}$ couplings turns the density operator into either $4 \mathrm{H}_{\mathrm{z}}^{\mathrm{N}} \mathrm{N}_{\mathrm{z}} \mathrm{C}_{\mathrm{y}}^{\prime}$ or $2 \mathrm{H}_{\mathrm{z}}^{\mathrm{N}} \mathrm{C}_{\mathrm{y}}^{\prime}$ prior to the $t_{1}$ period. Both the $\mathrm{C}^{\prime}$ chemical shifts and $\mathrm{C}^{\prime}$-N couplings evolve during the $t_{1}$ period. The term $4 \mathrm{H}_{\mathrm{z}}^{\mathrm{N}} \mathrm{N}_{\mathrm{z}} \mathrm{C}_{\mathrm{y}}^{\prime}$ yields an in-phase spectrum while $2 \mathrm{H}_{\mathrm{z}}^{\mathrm{N}} \mathrm{C}_{\mathrm{y}}^{\prime}$ gives an anti-phase spectrum. Addition and subtraction of the in- and anti-phase spectra generate two 3D sub-spectra. The relevant coordinates for extracting couplings along the $\mathrm{C}^{\prime}$ dimension in the two sub-spectra

are $\left\{\omega_{\mathrm{C}^{\prime}}+(1+\lambda) \pi^{1} \mathrm{~J}_{\mathrm{NC}^{\prime}}\right\}$ and $\left\{\omega_{\mathrm{C}^{\prime}}-(1+\lambda) \pi^{1} \mathrm{~J}_{\mathrm{NC}^{\prime}}\right\}$, respectively. The parameter $\lambda$ is used to amplify $\mathrm{C}^{\prime}-\mathrm{N}$ couplings in order to enhance the precision of measurement. The application of these experiments at high field spectrometers is limited by the fast $T_{2}$ relaxation of $C^{\prime}$. The authors suggested carrying out these experiments at a field strength of $600 \mathrm{MHz}$ proton frequency or lower. Analysis showed that the J-crosstalk does not significantly affect the precision of peak positions. The precision of the experiments was about 0.1 $\mathrm{Hz}$ when tested on $1.4 \mathrm{mM}{ }^{15} \mathrm{~N} /{ }^{13} \mathrm{C}$-labeled human cardiac Troponin $\mathrm{C}(\mathrm{cTnC}, 18 \mathrm{kDa})$.

A 3D TROSY-HNCO quantitative $\mathrm{J}_{\mathrm{C}^{\prime}-\mathrm{N}}$ experiment has been developed by Chou and coworkers [99]. Compared to a regular TROSY-HNCO experiment [92], a long delay of $2 \mathrm{~T}=66.7 \mathrm{~ms}$ is used in its INEPT step to transfer magnetization from ${ }^{15} \mathrm{~N}$ to $\mathrm{C}^{\prime}$. Two experiments are carried out in an interleaved manner with the positions of the $180^{\circ}$ pulse on $\mathrm{C}^{\prime}$ located at two different places. The signal intensities of the two experiments are modulated by $\mathrm{C}^{\prime}-\mathrm{N}$ couplings with different durations. Thus, $\mathrm{C}^{\prime}-\mathrm{N}$ couplings can be extracted from the intensity ratio of the two experiments. Details for data analysis can be found in the original work. The sequence has been demonstrated on a $1.0 \mathrm{mM}{ }^{13} \mathrm{C} /{ }^{15} \mathrm{~N}$-labeled $\mathrm{Ca}^{2+}$-mammalian calmodulin $(\mathrm{CaM}, 17$ $\mathrm{kDa}$ ) sample. The RMSD between the measured and predicted dipolar couplings is $0.21 \mathrm{~Hz}$. The precision of the measured couplings is proportional to the $\mathrm{S} / \mathrm{N}$ ratios.

\section{6 ${ }^{1} \mathrm{H}-{ }^{1} \mathrm{H}$ dipolar coupling}

Residual dipolar couplings between ${ }^{1} \mathrm{H}_{-}{ }^{1} \mathrm{H}$ can be observed and applied as long-range conformational constraints [4] for structure determination. Compared to traditional NOE distance constraints, ${ }^{1} \mathrm{H}-{ }^{1} \mathrm{H}$ RDCs can potentially provide distance constraints longer than $5 \AA$ since ${ }^{1} \mathrm{H}-{ }^{1} \mathrm{H}$ RDCs are proportional to $r^{-3}$ while NOEs 
are proportional to $r^{-6}$. Nevertheless, the overwhelming ${ }^{1} \mathrm{H}-{ }^{1} \mathrm{H}$ dipolar coupling network, especially those neighboring protons reduce greatly the values of ${ }^{1} \mathrm{H}-{ }^{1} \mathrm{H}$ RDCs. It is also difficult to assign the sign of remote ${ }^{1} \mathrm{H}^{1}{ }^{1} \mathrm{H}$ RDCs if there is no scalar couplings between them, or the magnitude of their scalar couplings are smaller than that of RDCs. Although the absolute values of ${ }^{1} \mathrm{H}-{ }^{1} \mathrm{H}$ RDCs can be used in structural study, RDCs with known sign can speed up the convergence in structure calculation [100]. Two approaches have been proposed to determine the sign of ${ }^{1} \mathrm{H}_{-}{ }^{1} \mathrm{H}$ RDCs for the spin system of $\mathrm{X}-{ }^{1} \mathrm{H} \leftrightarrow{ }^{1} \mathrm{H}^{\mathrm{r}}$, where $\mathrm{X}$ can be either ${ }^{13} \mathrm{C}$ or ${ }^{15} \mathrm{~N}, r$ stands for a remote proton [101, 102]. Another experiment, called soft HNCA-E.COSY (see section 3.7), can determine both the magnitude and sign of ${ }^{3} \mathrm{D}_{\mathrm{HN}-\mathrm{HA}}$ and ${ }^{4} \mathrm{D}_{\mathrm{HN}-\mathrm{HA}}$ [103].

DiM (dipolar couplings in methyls) is a $2 \mathrm{D}\left[{ }^{1} \mathrm{H},{ }^{13} \mathrm{C}\right]$-correlated experiment for measuring ${ }^{1} \mathrm{H}-{ }^{1} \mathrm{H}$ couplings in methyl groups [75]. Theoretical analysis shows that for an isolated methyl group, dipolar couplings result in a triplet with relative line intensities of $1: 2: 1$. The experiment was designed to detect the two outermost lines of the triplet, and suppress the middle one by creating a ${ }^{1} \mathrm{H}-{ }^{1} \mathrm{H}$ anti-phase term right before the data acquisition. The final cross peaks are proportional to $\sin \left(\pi \mathrm{D}_{\mathrm{HH}} \mathrm{t}_{2}\right) \cos \left(\pi \mathrm{D}_{\mathrm{HH}} \mathrm{t}_{2}\right)$. Thus, the two outermost lines of the triplet along the ${ }^{1} \mathrm{H}$ dimension yield $2 \mathrm{D}_{\mathrm{HH}}$, while the sign of the $\mathrm{D}_{\mathrm{HH}}$ is also shown in $\sin \left(\pi \mathrm{D}_{\mathrm{HH}} \mathrm{t}_{2}\right)$. The experiment has been demonstrated on $10 \mathrm{mM}$ BPTI at natural isotopic abundance. The pulse scheme should also be useful for perdeuterated proteins with protonated methyl groups $[75,1]$, because such labeling pattern would suppress the scalar and dipolar interaction between methyl and non-methyl protons.

An ${ }^{15} \mathrm{~N}$-resolved CT-COSY experiment [104] with enhanced spectrum resolution has been extended from an earlier 2D CT-COSY experiment [105] for the measurement of ${ }^{1} \mathrm{H}-{ }^{1} \mathrm{H}_{\mathrm{N}}$ couplings. ${ }^{15} \mathrm{~N}$ evolution is added in the constant time period, which is also used for ${ }^{1} \mathrm{H}_{\mathrm{N}}$ chemical shift labeling and the magnetization transfer from ${ }^{1} \mathrm{H}_{\mathrm{N}}$ to other proximate protons. The ${ }^{1} \mathrm{H}-{ }^{1} \mathrm{H}$ couplings can be extracted from the ratios of the cross-peaks to auto-peaks in an ${ }^{15} \mathrm{~N}$-resolved $2 \mathrm{D}\left[{ }^{1} \mathrm{H},{ }^{1} \mathrm{H}\right]$ plane. The pulse sequence has been tested on rubredoxin $(5.9 \mathrm{kDa})$ and $E$. coli acyl carrier protein (ACP, $8.8 \mathrm{kDa})$. The quality of the measured ${ }^{3} \mathrm{~J}_{\mathrm{HN}-\mathrm{H} \alpha}$ values on rubredoxin has been evaluated by a comparison with the data measured from an HNHA experiment [100], the RMSD between these two data sets is $0.6 \mathrm{~Hz}$.

A 3D SS-HMQC2 (two semi-selective HMQC) experiment has been developed by Wu and workers [106] for measuring long-range ${ }^{1} \mathrm{H}_{\mathrm{N}}-{ }^{1} \mathrm{H}_{\mathrm{N}}$ couplings. For ease of exposition, let $\mathrm{P}$ and $\mathrm{Q}$ denote two pairs of ${ }^{1} \mathrm{H}_{-}{ }^{15} \mathrm{~N}$ spin vectors. The experiment is composed of two constant-time (CT) periods. In the first CT period, the ${ }^{15} \mathrm{~N}^{\mathrm{P}}$ chemical shifts evolve and the magnetization of ${ }^{1} \mathrm{H}_{\mathrm{N}}^{\mathrm{P}}$ is transferred partially and selectively to other nearby ${ }^{1} \mathrm{H}_{\mathrm{N}}$ protons, say ${ }^{1} \mathrm{H}_{\mathrm{N}}^{\mathrm{Q}}$. The selective transfer is achieved by a $180^{\circ}$ selective pulse on ${ }^{1} \mathrm{H}_{\mathrm{N}}$. Then, the magnetization is relayed to ${ }^{15} \mathrm{~N}^{\mathrm{Q}}$ through ${ }^{1} \mathrm{H}_{\mathrm{N}}^{\mathrm{Q}}$. During the second CT period, the ${ }^{15} \mathrm{~N}$ chemical shifts evolve and anti-phase terms of the two ${ }^{1} \mathrm{H}_{\mathrm{N}}$ protons are rephased. The experiment is basically a ${ }^{15} \mathrm{~N}-{ }^{15} \mathrm{~N}$ COSY experiment correlated by ${ }^{1} \mathrm{H}_{\mathrm{N}}-{ }^{1} \mathrm{H}_{\mathrm{N}} \mathrm{RDC}$ interaction. Finally, the magnetization is transferred back to the attached ${ }^{1} \mathrm{H}_{\mathrm{N}}$ and detected. Thus, in a $2 \mathrm{D}\left[{ }^{15} \mathrm{~N},{ }^{15} \mathrm{~N}\right]$ plane, the intensities of both cross and diagonal peaks contain RDC information between their attached two ${ }^{1} \mathrm{H}_{\mathrm{N}}$ protons. The ${ }^{1} \mathrm{H}_{\mathrm{N}}-{ }^{1} \mathrm{H}_{\mathrm{N}} \mathrm{RDCs}$ can be extracted readily (see the original paper). The sequence has been demonstrated on $1.0 \mathrm{mM}{ }^{13} \mathrm{C} /{ }^{15} \mathrm{~N} /{ }^{2} \mathrm{H}$-labeled $\mathrm{Ca}^{2+}$-calmodulin, and cross peaks can be observed between two ${ }^{1} \mathrm{H}_{\mathrm{N}}$ protons up to $6 \AA$ apart. When tested on a ubiquitin sample, the RDC interaction between two ${ }^{1} \mathrm{H}_{\mathrm{N}}$ protons can be observed with distance up to $7 \AA$. Although only demonstrated on the ${ }^{1} \mathrm{H}_{\mathrm{N}}-{ }^{1} \mathrm{H}_{\mathrm{N}} \mathrm{RDC}$ measurement, the strategy used in the sequence should be applicable to other groups of protons. The method can not determine the sign of $\mathrm{D}_{\mathrm{HN}-\mathrm{HN}}$.

2D $\mathrm{D}_{\mathrm{HH}}$-CT-HSQC and 3D $\mathrm{D}_{\mathrm{HH}}-\mathrm{CT}-\mathrm{HSQC}$ experiments measure ${ }^{1} \mathrm{H}-{ }^{1} \mathrm{H}$ dipolar couplings in $\mathrm{CH}_{2} \mathrm{D}$ methyl groups of highly deuterated ${ }^{13} \mathrm{C} /{ }^{15} \mathrm{~N}$-labeled proteins [107]. $\mathrm{D}_{\mathrm{HH}}$ in methyl group provides useful information for defining $\chi_{1}$ torsion angles and stereo-specific assignment of methyl groups in valine side chains [107]. Such restraints are particular useful for perdeuterated proteins where no sufficient NOE distance 
restraints are available for defining methyl groups. In order to selectively observe the signals from $\mathrm{CH}_{2} \mathrm{D}$ groups, the signals from $\mathrm{CHD}_{2}$ and $\mathrm{CH}_{3}$ groups are suppressed through isotopomer selection filters. In addition, an $\alpha / \beta$ spin-state-selection step (using $\mathrm{J}_{\mathrm{CH}}$ ) is used before the final INEPT to enhance the resolution. Through addition and subtraction of IPAP spectra, two subspectra $(\alpha / \beta)$ are generated with the frequencies along the ${ }^{1} \mathrm{H}$ dimension at $\omega_{\beta}=\omega_{\mathrm{H}}+\mathrm{D}_{\mathrm{HH}} / 2$ and $\omega_{\alpha}=\omega_{\mathrm{H}}-\mathrm{D}_{\mathrm{HH}} / 2$, respectively. Since there is no scalar coupling between two protons in methyl groups, no reference spectrum is needed to extract the RDCs. For large-sized proteins, the $2 \mathrm{D}$ experiment can be extended to $3 \mathrm{D}$ by labeling the ${ }^{13} \mathrm{C}$ next to the methyl ${ }^{13} \mathrm{C}$ using an out-and-back method. Both $2 \mathrm{D}$ and $3 \mathrm{D}$ pulse schemes have been demonstrated on ${ }^{13} \mathrm{C} /{ }^{15} \mathrm{~N}$, and $77 \%$ ${ }^{2} \mathrm{H}$-labeled SiR-FP18 protein.

\subsection{Experiments for the simultaneous measurement of more than one types of RDCs}

In above sections, we have discussed experiments for measuring one type of RDCs at a time. There are several advantages for the simultaneous measurement of different types of RDCs using a single experiment [108, 109, 110]:

1. In high-throughput biological structure determination, it is preferred to obtain as many types of RDCs as possible in a timely-fashion $[111,109]$.

2. In practice, the conditions of alignment samples may change in the course of measuring different types of RDCs in a sequential manner. The changes can be caused by (a) different alignments since it may take a long time for reaching the equilibrium alignment state [110], (b) the instability of alignment samples, and (c) different NMR experimental conditions such as pulse imperfection and heating associated with different pulse sequences. Consequently, the different types of sequentially-measured RDCs may not share a common alignment tensor, which introduces additional errors in RDC applications.

However, compared with the spectra of only one type of RDCs, the spectra of several types of RDCs are more crowded and their S/N ratios are lower. In general, E.COSY schemes [42] have been used for enhancing resolution and TROSY techniques for increasing $\mathrm{S} / \mathrm{N}$ ratios. For examples, the couplings of ${ }^{1} \mathrm{H}_{\mathrm{N}}-{ }^{13} \mathrm{C}^{\prime}$, ${ }^{15} \mathrm{~N}-{ }^{13} \mathrm{C}^{\prime}$ and ${ }^{1} \mathrm{H}-{ }^{15} \mathrm{~N}$ can be measured using $\left[{ }^{1} \mathrm{H},{ }^{15} \mathrm{~N}\right]$-HSQC or -TROSY type experiments in combination with E.COSY schemes:

1. 2D E.COSY-IPAP-HSQC for simultaneous measurement of RDCs of ${ }^{1} \mathrm{H}_{\mathrm{N}}-{ }^{13} \mathrm{C}^{\prime},{ }^{15} \mathrm{~N}-{ }^{13} \mathrm{C}^{\prime}$ and ${ }^{1} \mathrm{H}$ ${ }^{15} \mathrm{~N}$ for large-sized perdeuterated proteins [112].

2. $2 \mathrm{D} \mathrm{HN}\left(\alpha / \beta\right.$-NC'-J)-HSQC and $\mathrm{HN}\left(\alpha / \beta\right.$-NC'-J)-TROSY for measuring ${ }^{1} \mathrm{H}_{\mathrm{N}}-{ }^{13} \mathrm{C}^{\prime}$ and ${ }^{15} \mathrm{~N}-{ }^{13} \mathrm{C}^{\prime}$ couplings [98], as well as the couplings of ${ }^{15} \mathrm{~N}-{ }^{13} \mathrm{C}^{\prime}$ and ${ }^{1} \mathrm{H}_{\mathrm{N}}-{ }^{13} \mathrm{C}^{\prime}$ in Asn and Gln side chains with an $\mathrm{NH}_{2}$ selection filter [81];

3. 2D $\left(\delta\right.$, J)-E.COSY HSQC for measuring ${ }^{1} \mathrm{H}_{-}{ }^{15} \mathrm{~N},{ }^{15} \mathrm{~N}-{ }^{13} \mathrm{C}^{\prime}$ and ${ }^{1} \mathrm{H}_{\mathrm{N}}-{ }^{13} \mathrm{C}^{\prime}$ couplings [78, 113].

4. 3D E.COSY-HNCO-TROSY experiment for measuring ${ }^{1} \mathrm{H}_{\mathrm{N}}-{ }^{13} \mathrm{C}^{\prime}$ and ${ }^{15} \mathrm{~N}-{ }^{13} \mathrm{C}^{\prime}$ couplings on largesized proteins [77].

In addition to the experiments listed above, many different experiments have been proposed. It is difficult to group them based on the types of RDCs since each experiment measures a different combination of RDCs. We will discuss the experiments in the order of publication date. For easy of reference, in the following the measured RDCs are listed right after the name of the experiment.

$\left[{ }^{1} \mathrm{H}^{13} \mathrm{C}\right]-\mathrm{CT}-\mathrm{HSQC}$ measures $\mathrm{J}_{\mathrm{CH}}$ couplings of methane, methylene and methyl groups [114]. It is an extension of CT-HSQC with the third dimension modulated with active $\mathrm{J}_{\mathrm{CH}}$ couplings. The constant-time duration is set to be $\frac{1}{J_{C C}} \approx 28 \mathrm{~ms}$. The dwell time for $\mathrm{J}_{\mathrm{CH}}$ modulation ( $t_{2}$ dimension) is about $2 \mathrm{~ms}$, thus less than 15 increments are needed for the $\mathrm{J}$ modulation. The individual $2 \mathrm{D}\left[{ }^{1} \mathrm{H},{ }^{13} \mathrm{C}\right]$ planes appear like a regular decoupled CT-HSQC. The intensity depends on the $t_{2}$ duration with cosine functions for methane, methylene 
and methyl groups. The coupling interactions between carbon and remote protons are accounted for the decay of the signal in the $t_{2}$ dimension. Although only the sum of the two $\mathrm{J}_{\mathrm{CH}}$ splittings can be determined for methylene sites, RDCs of the sum can still be used for structure calculation. For methyl groups, the dipolar couplings are measured from the outer ${ }^{13} \mathrm{C}$ quartet components. These outer quartet components are modulated by $3 \pi\left({ }^{1} \mathrm{~J}_{\mathrm{CH}}+{ }^{1} \mathrm{D}_{\mathrm{CH}}\right)$, and thus the RDC can be measured accurately although it is scaled down by a factor of -0.31 due to the rapid rotation about a three-fold symmetry axis [115]. The RMSD of measured RDCs from ${ }^{13} \mathrm{C}$ inner and outer quatet components of methyl group is less than $0.3 \mathrm{~Hz}$. The pairwise RMSD is less than $0.4 \mathrm{~Hz}$ for methylene sites. The sequence has been tested on $0.7 \mathrm{mM}{ }^{13} \mathrm{C} /{ }^{15} \mathrm{~N}$-labeled ubiquitin, and should be applicable to small and medium-sized proteins.

3D E.COSY-HNCO-TROSY measures RDCs of $\mathrm{H}_{\mathrm{N}}(\mathrm{i})-\mathrm{C}_{\alpha}(\mathrm{i}-1)$ and $\mathrm{C}^{\prime}(\mathrm{i}-1)-\mathrm{C}_{\alpha}(\mathrm{i}-1)$ internuclear vectors [116]. To establish an E.COSY pattern, both $\mathrm{C}^{\prime}(\mathrm{i}-1)-\mathrm{C}_{\alpha}(\mathrm{i}-1)$ and $\mathrm{H}_{\mathrm{N}}(\mathrm{i})-\mathrm{C}_{\alpha}(\mathrm{i}-1)$ couplings are active during the ${ }^{13} \mathrm{C}^{\prime}$ evolution $\left(t_{1}\right)$ and ${ }^{1} \mathrm{H}$ acquisition $\left(t_{3}\right)$ periods. The $\alpha$ and $\beta$ spin states of $\mathrm{C}_{\alpha}$ are not mixed from the $t_{1}$ to $t_{3}$ periods. Thus, E.COSY patterns of doublets appear in the plane of $\left[{ }^{13} \mathrm{C}^{\prime},{ }^{1} \mathrm{H}_{\mathrm{N}}\right]$ with relevant coordinates at $\left\{\omega_{\mathrm{C}^{\prime}}+\pi^{1} \mathrm{~J}_{\mathrm{C}^{\prime} \mathrm{C} \alpha}, \omega_{\mathrm{HN}}-\pi^{3} \mathrm{~J}_{\mathrm{HN}-\mathrm{C} \alpha}\right\}$ and $\left\{\omega_{\mathrm{C}^{\prime}}-\pi^{1} \mathrm{~J}_{\mathrm{C}^{\prime} \mathrm{C} \alpha}, \omega_{\mathrm{HN}}+\pi^{3} \mathrm{~J}_{\mathrm{HN}-\mathrm{C} \alpha}\right\}$, and from them the couplings of $\mathrm{H}_{\mathrm{N}}(\mathrm{i})-\mathrm{C}_{\alpha}(\mathrm{i}-1)$ and $\mathrm{C}^{\prime}(\mathrm{i}-1)-\mathrm{C}_{\alpha}(\mathrm{i}-1)$ can be extracted readily. The reproducibility of coupling measurement has been evaluated on a $1 \mathrm{mM}$ isotropic MBP sample with repeated experiments. The average values for ${ }^{1} \mathrm{~J}_{\mathrm{C}^{\prime}-\mathrm{C} \alpha}$ and ${ }^{3} \mathrm{~J}_{\mathrm{HN}-\mathrm{C} \alpha}$ are, respectively, $52.5 \pm 1.0$ and $0.3 \pm 0.4 \mathrm{~Hz}$ based on the values from 275 well-resolved cross peaks in the spectra. This approach can be further improved in resolution by inserting a spin-state-filter to separate the doublets of $\mathrm{C}^{\prime}-\left\{\mathrm{C}_{\alpha}\right\}$ into two sub-spectra as demonstrated in 3D $\mathrm{HNCO}\left(\alpha / \beta-\mathrm{C}^{\prime} \mathrm{C}_{\alpha}-\mathrm{J}\right)$ and 3D $\mathrm{HNCO}\left(\alpha / \beta-\mathrm{C}^{\prime} \mathrm{C}_{\alpha}-\mathrm{J}\right)$-TROSY experiments [97].

Soft HNCA-E.COSY measures RDCs of $\mathrm{H}_{\alpha}(\mathrm{i})-\mathrm{C}_{\alpha}(\mathrm{i}), \mathrm{H}_{\mathrm{N}}(\mathrm{i})-\mathrm{H}_{\alpha}(\mathrm{i})$ and $\mathrm{H}_{\mathrm{N}}(\mathrm{i})-\mathrm{H}_{\alpha}(\mathrm{i}-1)$ internuclear vectors [103]. The experiment is modified from an earlier version of HNCA-E.COSY sequence [117]. The coherence is transferred as follows:

$$
{ }^{1} \mathrm{H}_{\mathrm{N}}(\mathrm{i}) \rightarrow{ }^{15} \mathrm{~N}(\mathrm{i})\left(\mathrm{CT}, \mathrm{t}_{2}\right) \rightarrow{ }^{13} \mathrm{C}_{\alpha}(\mathrm{i})\left(\mathrm{t}_{1}\right) / \mathrm{C}_{\alpha}(\mathrm{i}-1)\left(\mathrm{t}_{1}\right) \rightarrow{ }^{15} \mathrm{~N}(\mathrm{i}) \rightarrow{ }^{1} \mathrm{H}_{\mathrm{N}}(\mathrm{i})\left(\mathrm{t}_{3}\right)
$$

The duration of CT for the magnetization transfer from ${ }^{15} \mathrm{~N}$ to $\mathrm{C}_{\alpha}$ is $27.2 \mathrm{~ms}$. This CT period is also used for the ${ }^{15} \mathrm{~N}$ chemical shift evolution. Both the chemical shifts of $\mathrm{C}_{\alpha}(\mathrm{i})$ and $\mathrm{C}_{\alpha}(\mathrm{i}-1)$, and their couplings to the ${ }^{1} \mathrm{H}_{\alpha}(\mathrm{i})$ evolve during the $t_{1}$ period. When the magnetization is transferred back to ${ }^{1} \mathrm{H}_{\mathrm{N}}$ from ${ }^{15} \mathrm{~N}$, a net $360^{\circ}$ rotation of ${ }^{1} \mathrm{H}_{\alpha}$ keeps its spin state the same as that during the $\mathrm{C}_{\alpha}$ evolution time. The couplings between $\mathrm{H}_{\mathrm{N}}$ and $\mathrm{H}_{\alpha}(\mathrm{i}) / \mathrm{H}_{\alpha}(\mathrm{i}-1)$ are active during the data acquisition. The cross peaks in $\left[{ }^{13} \mathrm{C},{ }^{1} \mathrm{H}_{\mathrm{N}}\right]$ planes form E.COSY patterns, and the large ${ }^{1} \mathrm{~J}_{\mathrm{C}_{\alpha} \mathrm{H}_{\alpha}}$ coupling makes the small couplings of ${ }^{1} \mathrm{H}_{\alpha^{-}}{ }^{1} \mathrm{H}_{\mathrm{N}}$ resolved. Consequently, the couplings of $\mathrm{H}_{\alpha}(\mathrm{i})-\mathrm{C}_{\alpha}(\mathrm{i}), \mathrm{H}_{\mathrm{N}}(\mathrm{i})-\mathrm{H}_{\alpha}(\mathrm{i})$ and $\mathrm{H}_{\mathrm{N}}(\mathrm{i})-\mathrm{H}_{\alpha}(\mathrm{i}-1)$ can be measured readily. When tested on a FK506 binding protein complexed to FK506, $90 \%$ of the possible $\mathrm{H}_{\mathrm{N}}(\mathrm{i})-\mathrm{H}_{\alpha}(\mathrm{i}) \mathrm{RDCs}$ and $52 \%$ of the possible $\mathrm{H}_{\mathrm{N}}(\mathrm{i})-\mathrm{H}_{\alpha}(\mathrm{i}-1)$ RDCs could be obtained.

The ${ }^{1} \mathrm{D}_{\mathrm{N}-\mathrm{C} \alpha},{ }^{2} \mathrm{D}_{\mathrm{N}-\mathrm{C} \alpha},{ }^{2} \mathrm{D}_{\mathrm{HN}-\mathrm{C} \alpha}$ and ${ }^{3} \mathrm{D}_{\mathrm{HN}-\mathrm{C} \alpha}$ RDCs can be measured by either $2 \mathrm{D} \mathrm{HN}\left(\mathrm{CO}-\alpha / \beta-\mathrm{NC}_{\alpha}-\right.$ J)-TROSY or $3 \mathrm{D}-\mathrm{HNCO}\left(\alpha / \beta-\mathrm{NC}_{\alpha}-\mathrm{J}\right)$-TROSY $[97,71]$. It is challenging to measure ${ }^{1} \mathrm{D}_{\mathrm{N}-\mathrm{C} \alpha}$ and ${ }^{2} \mathrm{D}_{\mathrm{N}-\mathrm{C} \alpha}$ since they are small and difficult to be resolved from one another. The proposed experiments make use of large ${ }^{1} \mathrm{~J}_{\mathrm{C}^{\prime}-\mathrm{C} \alpha}$ coupling to distinguish the inter/intra-residue couplings of $\mathrm{N}-\mathrm{C}_{\alpha}$. The coherence is transferred in $2 \mathrm{D}$ $\mathrm{HN}\left(\mathrm{CO}-\alpha / \beta-\mathrm{NC}_{\alpha}-\mathrm{J}\right)-\mathrm{TROSY}$ as follows:

$$
{ }^{1} \mathrm{H}_{\mathrm{N}} \rightarrow{ }^{15} \mathrm{~N} \rightarrow{ }^{13} \mathrm{C}^{\prime} \rightarrow{ }^{15} \mathrm{~N}\left(\mathrm{t}_{1}\right) \rightarrow{ }^{1} \mathrm{H}_{\mathrm{N}}\left(\mathrm{t}_{2}\right)
$$

A spin-state-filter (using large ${ }^{1} \mathrm{~J}_{\mathrm{C}^{\prime}-\mathrm{C} \alpha}$ couplings) is inserted right after the magnetization has been transferred from ${ }^{15} \mathrm{~N}$ to $\mathrm{C}^{\prime}$. Before the ${ }^{15} \mathrm{~N}$ evolution period, the filter generates a $4 \mathrm{H}_{\mathrm{z}} \mathrm{N}_{\mathrm{y}}(\mathrm{i}) \mathrm{C}_{\mathrm{z}}^{\prime}(\mathrm{i}-1)$ term for in-phase 


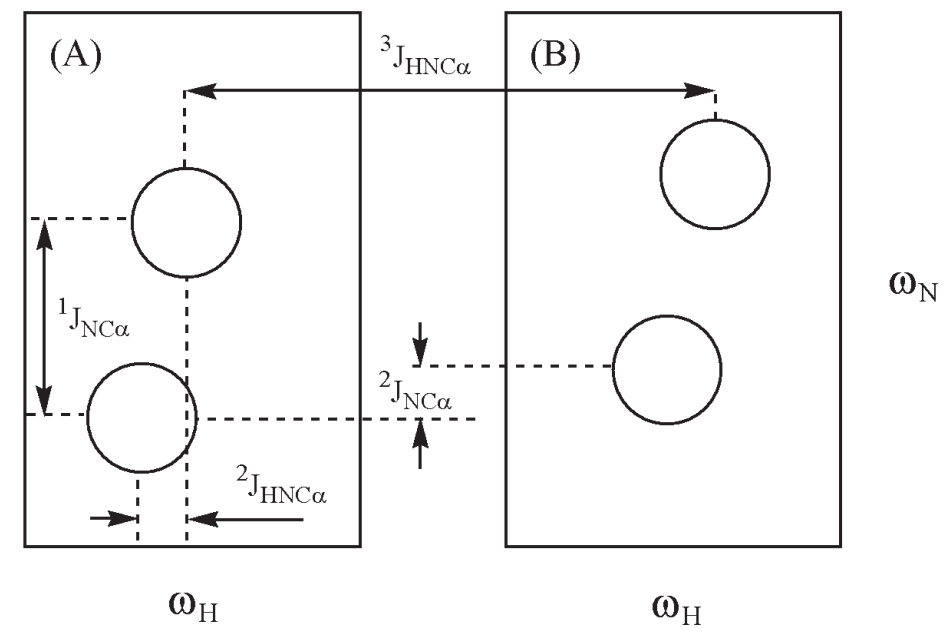

Figure 8: Two sub-spectra obtained from the sequence of $2 \mathrm{D} \mathbf{H N}\left(\mathrm{CO}-\alpha / \beta-\mathrm{NC}_{\alpha}-\mathrm{J}\right)-\mathbf{T R O S Y} .{ }^{1} \mathrm{~J}_{\mathrm{N}-\mathrm{C} \alpha}$ and ${ }^{2} \mathrm{~J}_{\mathrm{HN}-\mathrm{C} \alpha}$ couplings can be measured twice from E.COSY patterns within the panels of (A) and (B), while ${ }^{2} \mathrm{~J}_{\mathrm{N}-\mathrm{C} \alpha}$ and ${ }^{3} \mathrm{~J}_{\mathrm{HN}-\mathrm{C} \alpha}$ couplings can be measured twice from the corresponding components in two sub-spectra.

experiment and a $8 \mathrm{H}_{\mathrm{z}} \mathrm{N}_{\mathrm{y}}(\mathrm{i}) \mathrm{C}_{\mathrm{z}}^{\prime}(\mathrm{i}-1) \mathrm{C}_{\mathrm{z}}^{\alpha}(\mathrm{i}-1)$ term for anti-phase experiment. During the $t_{1}$ evolution, these two terms yield in-phase and anti-phase spectra for the doublets of ${ }^{15} \mathrm{~N}(\mathrm{i})-\left\{\mathrm{C}_{\alpha}(\mathrm{i}-1)\right\}$, respectively. These doublets are further split by the couplings to $\mathrm{C}_{\alpha}(\mathrm{i})$. Finally, the couplings between $\mathrm{C}_{\alpha}$ and ${ }^{1} \mathrm{H}_{\mathrm{N}}$ are active during the data $\left({ }^{1} \mathrm{H}_{\mathrm{N}}\right)$ acquisition. Since the $\mathrm{C}_{\alpha}$ spin states are preserved and coupled to both ${ }^{15} \mathrm{~N}$ and ${ }^{1} \mathrm{H}_{\mathrm{N}}$ dimensions, an E.COSY pattern forms. The linear combination of the in-phase and anti-phase spectra generates two sub-spectra, where the ${ }^{1} \mathrm{~J}_{\mathrm{N}-\mathrm{C} \alpha}$ and ${ }^{2} \mathrm{~J}_{\mathrm{HN}-\mathrm{C} \alpha}$ couplings can be measured directly from the E.COSY pattern within each sub-spectra. The ${ }^{2} \mathrm{~J}_{\mathrm{N}-\mathrm{C} \alpha}$ and ${ }^{3} \mathrm{~J}_{\mathrm{HN}-\mathrm{C} \alpha}$ couplings can be extracted from the frequency difference of corresponding peaks in these two spectra. Shown in Fig. 8 are the cross peaks of two sub-spectra illustrating the strategies for extracting the couplings. All the four couplings can be determined twice, and thus provide an internal check for the quality of measurement. The sequence has been demonstrated on 1.0 $\mathrm{mM}{ }^{13} \mathrm{C} /{ }^{15} \mathrm{~N}$-labeled ubiquitin. For large-sized protein samples, a third dimension $\left({ }^{13} \mathrm{C}^{\prime}\right)$ can be added to enhance the resolution [97]. Later on, a similar $2 \mathrm{D}\left[{ }^{15} \mathrm{~N},{ }^{1} \mathrm{H}\right]$ correlation experiment has been proposed for measuring the same four couplings, ${ }^{1} \mathrm{~J}_{\mathrm{N}-\mathrm{C} \alpha},{ }^{2} \mathrm{~J}_{\mathrm{N}-\mathrm{C} \alpha},{ }^{2} \mathrm{~J}_{\mathrm{HN}-\mathrm{C} \alpha}$ and ${ }^{3} \mathrm{~J}_{\mathrm{HN}-\mathrm{C} \alpha}$ [118]. The experiment is based on a sensitivity-enhanced HNCO rather than a TROSY scheme, this approach is more suitable for small-sized proteins (less than $10 \mathrm{kDa}$, or the ${ }^{15} \mathrm{~N}$ linewidth is less than $8.5 \mathrm{~Hz}$ ). The sequence had been tested on protein GB1 $(6.2 \mathrm{kDa})$.

SPITZE-HSQC (SPIn sTate selective Zero overlap HSQC) measures ${ }^{2} \mathrm{~J}_{\mathrm{H}-\mathrm{H}}$ and two individual couplings of C-H bonds in a methylene group [119]. SPITZE-HSQC is a fully coupled HSQC experiment in both ${ }^{13} \mathrm{C}$ and ${ }^{1} \mathrm{H}$ dimensions. Along the ${ }^{13} \mathrm{C}$ dimension, only two outer components are observable, while the middle component is canceled. Each of these two outer components is further split into four components along the ${ }^{1} \mathrm{H}$ dimension by the one-bond $\mathrm{C}-\mathrm{H}$ and two-bond $\mathrm{H}-\mathrm{H}$ couplings. Using an $\mathrm{S}^{3} \mathrm{E}$ scheme [39, 40], four out of the eight components containing information of the three couplings can be selectively observed from four individual experiments. Thus, all the three RDCs of $\mathrm{CH}^{1}, \mathrm{CH}^{2}$ and $\mathrm{H}-\mathrm{H}$ for moiety of methylene can be determined. The combination of these three RDCs can be used to define the orientation of methylene groups [119]. The experiment is quite sensitive since the coherence is transferred selectively for each observed component. The sequence works well on Gly residue. The applications to other residues are compromised because the carbon-carbon couplings are active during the $t_{1}$ evolution. The pulse scheme has been demonstrated on a 1.5 $\mathrm{mM}{ }^{13} \mathrm{C} /{ }^{15}$ Nlabeled ubiquitin sample. 
3D CBCA(CO)NH measures the couplings of ${ }^{13} \mathrm{C}_{\alpha^{-}}{ }^{1} \mathrm{H}_{\alpha}$ and ${ }^{13} \mathrm{C}_{\beta^{-}}{ }^{1} \mathrm{H}_{\beta}$ bonds [120]. The experiment is modified from an original $\mathrm{CBCA}(\mathrm{CO}) \mathrm{NH}$ pulse sequence [121]. The magnetization transfer pathway is as follows:

$$
{ }^{1} \mathrm{H}_{\alpha / \beta} \rightarrow{ }^{13} \mathrm{C}_{\alpha / \beta}\left(\mathrm{CT}, \mathrm{t}_{1}\right) \rightarrow{ }^{13} \mathrm{C}^{\prime} \rightarrow{ }^{15} \mathrm{~N}\left(\mathrm{t}_{2}\right) \rightarrow{ }^{1} \mathrm{H}_{\mathrm{N}}\left(\mathrm{t}_{3}\right)
$$

In order to measure couplings of both ${ }^{13} \mathrm{C}_{\alpha^{-}}{ }^{1} \mathrm{H}_{\alpha}$ and ${ }^{13} \mathrm{C}_{\beta^{-}}{ }^{1} \mathrm{H}_{\beta}$, a quantitative-J modulation is introduced in the constant time period ( $t_{1}$ evolution). Three data sets are acquired in an interleaved manner where the signal intensities are modulated by $\mathbf{J}_{\mathrm{CH}}$ with three different time periods $2 \Delta_{1}$. Without considering cross-correlated relaxation effect, the signal intensities, $S\left(\Delta_{1}\right)$, associated with $\mathrm{C}_{\alpha}$ and $\mathrm{C}_{\beta}$ can be described by,

$$
S\left(\Delta_{1}\right)=A \sin \left[\pi \Sigma J_{C H}\left(2 \Delta_{1}+D_{e f f}\right)\right]
$$

where $A$ is a constant, $D_{\text {eff }}$ accounts for $\mathrm{J}_{\mathrm{CH}}$ dephasing due to various necessary delays and pulses, $\sum \mathrm{J}_{\mathrm{CH}}$ is the sum of the scalar couplings. The $\sum \mathrm{J}_{\mathrm{CH}}$ couplings can be derived from Eq. (13) by a SIMPLEX minimization routine using the three $2 \Delta_{1}$ values. Although only the sum of the two ${ }^{13} \mathrm{C}_{\beta^{-}}{ }^{1} \mathrm{H}_{\beta} \mathrm{RDCs}$ is available from the experiment, they are still useful for defining the $\chi_{1}$ dihedral angles of sidechain conformations. The first experiment is called reference experiment with $2 \Delta_{1}+D_{\text {eff }}=1.93 \mathrm{~ms}$, which is basically the same as a regular $\mathrm{CBCA}(\mathrm{CO}) \mathrm{NH}$ experiment. The $\mathrm{S} / \mathrm{N}$ ratio of the reference experiment determines the error in the coupling measurement. The sequence has been tested on $0.4 \mathrm{mM} \mathrm{Ca}^{2+}$-calmodulin.

A 3D HNCO-based experiment has been developed to simultaneously measure ${ }^{1} \mathrm{H}^{15}{ }^{15}$ and $\mathrm{C}^{\prime}-\mathrm{C}_{\alpha}$ couplings [108] by letting $\mathrm{J}_{\mathrm{C}^{\prime}} \mathrm{C}_{\alpha}$ to be active during the ${ }^{13} \mathrm{C}^{\prime}$ evolution and $\mathrm{J}_{\mathrm{HN}}$ to be active during the ${ }^{15} \mathrm{~N}$ evolution periods. An IPAP scheme [38] is inserted after the ${ }^{15} \mathrm{~N}$ evolution to enhance the resolution. Addition and subtraction of the IP and AP spectra result in two 3D spectra with cross peaks at $\left\{\omega_{\mathrm{C}^{\prime}} \pm \pi^{1} \mathrm{~J}_{\mathrm{C}^{\prime} \mathrm{C} \alpha}, \omega_{\mathrm{N}}+{ }^{1} \mathrm{~J}_{\mathrm{HN}}, \omega_{\mathrm{HN}}\right\}$ and $\left\{\omega_{\mathrm{C}^{\prime}} \pm \pi^{1} \mathrm{~J}_{\mathrm{C}^{\prime} \mathrm{C} \alpha}, \omega_{\mathrm{N}}-{ }^{1} \mathrm{~J}_{\mathrm{HN}}, \omega_{\mathrm{HN}}\right\} . \mathrm{J}_{\mathrm{C}^{\prime} \mathrm{C} \alpha}$ couplings can be measured twice from the two spectra. ${ }^{1} \mathrm{H}_{-}{ }^{15} \mathrm{~N}$ couplings can be extracted from the frequency displacement of the corresponding peaks along the ${ }^{15} \mathrm{~N}$ dimension in the two spectra. The experiment has been tested on an exclusive $\alpha$-helical protein, apoptotic inducing protein $\mathrm{Bax}(22 \mathrm{kDa})$. A regular 3D (HA)CA(CO)NH experiment has also been modified in a similar manner to measure, simultaneously, ${ }^{1} \mathrm{H}^{-15} \mathrm{~N}$ and ${ }^{13} \mathrm{C}_{\alpha^{-}}{ }^{1} \mathrm{H}_{\alpha}$ couplings [108]. The interested readers can refer to the original paper for the details.

A quantitative $\mathrm{HCN}-\mathrm{HSQC}$ experiment has been developed to measure long range (span more than three bonds) ${ }^{1} \mathrm{H}_{\mathrm{N}}-{ }^{13} \mathrm{C}$ couplings in perdeuterated proteins [122]. It is challenging to measure and assign such long range RDCs. The proposed experiment takes advantage of perdeuterated protein samples. The magnetization transfer pathway is as follows:

$$
{ }^{1} \mathrm{H}_{\mathrm{N}} \rightarrow{ }^{13} \mathrm{C}\left(\mathrm{t}_{1}\right) \rightarrow{ }^{1} \mathrm{H}_{\mathrm{N}} \rightarrow{ }^{15} \mathrm{~N}\left(\mathrm{t}_{2}\right) \rightarrow{ }^{1} \mathrm{H}_{\mathrm{N}}\left(\mathrm{t}_{3}\right)
$$

The duration (2T) for the transfer from ${ }^{1} \mathrm{H}_{\mathrm{N}}$ to ${ }^{13} \mathrm{C}$ and back was set to be $20 \mathrm{~ms}$. The ${ }^{13} \mathrm{C}$ can be either aliphatic or carbonyl ${ }^{13} \mathrm{C}$ nuclei in proximity of ${ }^{1} \mathrm{H}_{\mathrm{N}}$ spins. The intensity of the $3 \mathrm{D}$ spectrum is proportional

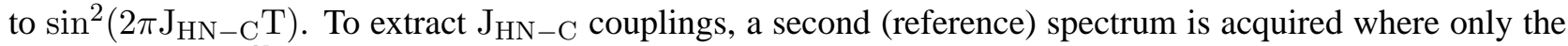
in-phase term ${ }^{1} \mathrm{H}_{\mathrm{z}}^{\mathrm{N}}$ after the first $2 \mathrm{~T}$ INEPT transfer period is selected, and a $2 \mathrm{D}\left[{ }^{15} \mathrm{~N},{ }^{1} \mathrm{H}\right]$ correlated spectrum is collected. The intensity of the signals is proportional to $\cos ^{2}\left(2 \pi \mathrm{J}_{\mathrm{HN}-\mathrm{C}} \mathrm{T}\right)$. With a suitable scaling factor to normalize the difference between the $2 \mathrm{D}$ and $3 \mathrm{D}$ acquisition schemes, the $\mathrm{J}_{\mathrm{HN}-\mathrm{C}}$ can be extracted from the intensity ratios of the cross and reference peaks. When tested on a $2.2 \mathrm{mM}{ }^{13} \mathrm{C} /{ }^{15} \mathrm{~N} /{ }^{2} \mathrm{H}$-labeled protein $\mathrm{G}$, many correlations between ${ }^{1} \mathrm{H}_{\mathrm{N}}$ and $\mathrm{C}_{\alpha}, \mathrm{C}_{\beta}$ and ${ }^{13} \mathrm{C}^{\prime}$ of both the intra or inter residues can be observed. In some cases, the correlation across a hydrogen bond $\left({ }^{1} \mathrm{H}_{\mathrm{N}} \leftrightarrow{ }^{13} \mathrm{C}^{\prime}\right)$ can be observed. In addition to the 
correlation of ${ }^{1} \mathrm{H}_{\mathrm{N}}$ and ${ }^{13} \mathrm{C}$, many correlations between ${ }^{1} \mathrm{H}_{\mathrm{N}}$ and ${ }^{1} \mathrm{H}_{\mathrm{N}}$ can also be observed using a regular ${ }^{1} \mathrm{H}-{ }^{1} \mathrm{H}$ COSY-HMQC [123], the distance of this correlation can span up to 7.2 $\AA$. The precision of the measurement has been evaluated, and the statistical error is smaller than $0.14 \mathrm{~Hz}$ for the measurement of $\mathrm{D}_{\mathrm{HN}-\mathrm{C}} \mathrm{RDCs}$.

A quantitative $\mathrm{J}$-correlated $\left[{ }^{15} \mathrm{~N},{ }^{1} \mathrm{H}\right]$-TROSY-HNC has been designed to measure ${ }^{1} \mathrm{~J}_{\mathrm{N}-\mathrm{C}^{\prime}},{ }^{1} \mathrm{~J}_{\mathrm{N}-\mathrm{C} \alpha}$ and ${ }^{2} \mathrm{~J}_{\mathrm{N}-\mathrm{C} \alpha}$ couplings [110]. The experiment is similar to an earlier TROSY-HNCO or TROSY-HNCA experiment [92]. The coherence transfer pathway is as follows:

$$
{ }^{1} \mathrm{H}_{\mathrm{N}} \rightarrow{ }^{15} \mathrm{~N} \rightarrow\left(\mathrm{C}^{\prime}(\mathrm{i}-1)\left(\mathrm{t}_{1}\right), \mathrm{C}_{\alpha}(\mathrm{i}-1)\left(\mathrm{t}_{1}\right), \mathrm{C}_{\alpha}(\mathrm{i})\left(\mathrm{t}_{1}\right), \mathrm{N}\left(\omega_{\mathrm{C}}=0\right)\right) \rightarrow{ }^{15} \mathrm{~N}\left(\mathrm{t}_{2}\right) \rightarrow{ }^{1} \mathrm{H}_{\mathrm{N}}\left(\mathrm{t}_{3}\right)
$$

The magnetization is first transferred, simultaneously, to $\mathrm{C}^{\prime}(\mathrm{i}-1), \mathrm{C}_{\alpha}(\mathrm{i}-1)$ and $\mathrm{C}_{\alpha}(\mathrm{i})$ using an $\mathrm{HMQC}$ scheme, then these three carbon nuclei evolve during the $t_{1}$ period and appear as cross peaks. In the meantime, the partial magnetization not transferred to ${ }^{13} \mathrm{C}$ from ${ }^{15} \mathrm{~N}$ is also sampled during the $t_{1}$ period through phase cyclings. These peaks appear as axial peaks since there exist no modulations from ${ }^{13} \mathrm{C}$ chemical shifts. Thus, both cross and axial peaks are in the same spectrum. The volume ratios of cross peaks versus axial peaks can be used to determine ${ }^{1} \mathrm{~J}_{\mathrm{N}-\mathrm{C}^{\prime}},{ }^{1} \mathrm{~J}_{\mathrm{N}-\mathrm{C} \alpha}$ and ${ }^{2} \mathrm{~J}_{\mathrm{N}-\mathrm{C} \alpha}$ couplings. Since the couplings between $\mathrm{C}^{\prime} / \mathrm{C}_{\alpha}$ and $\mathrm{C}_{\alpha} / \mathrm{C}_{\beta}$ are active during the $t_{1}$ period, the linewidths of cross peaks are broader than those of axial peaks. To avoid systematic errors, it is also critical to use peak volumes not peak intensities to compute these couplings. The experiments have been tested on three different proteins: ${ }^{13} \mathrm{C} /{ }^{15} \mathrm{~N}$-labeled flavodoxin $(16 \mathrm{kDa}),{ }^{13} \mathrm{C} /{ }^{15} \mathrm{~N}$-labeled xylanase $(23 \mathrm{kDa})$ and ${ }^{13} \mathrm{C} /{ }^{15} \mathrm{~N} /{ }^{2} \mathrm{H}$-labeled DFPase $(35 \mathrm{kDa})$. RDC values have been extracted on flavodoxin. The reproducibility of the experiments have been evaluated by acquiring two data sets on three isotropic and one aligned samples. The RMSD is less than $0.4 \mathrm{~Hz}$ for all the three couplings measured from the four different samples, while the uncertainties increase from ${ }^{1} \mathrm{~J}_{\mathrm{N}-\mathrm{C}^{\prime}}$ to ${ }^{1} \mathrm{~J}_{\mathrm{N}-\mathrm{C} \alpha}$ and to ${ }^{2} \mathrm{~J}_{\mathrm{N}-\mathrm{C} \alpha}$ couplings. The precision is proportional to the $\mathrm{S} / \mathrm{N}$ ratio of cross peaks. It is interesting that authors compared the RMSDs measured from this experiment with those measured from two other experiments: a $2 \mathrm{D} \mathrm{HN}(\alpha / \beta$-NC' $-\mathrm{J})$-TROSY (measures $\mathrm{N}-\mathrm{C}^{\prime}$ couplings) and $\mathrm{HN}\left(\mathrm{CO}-\alpha / \beta-\mathrm{NC}_{\alpha}-\mathrm{J}\right)$-TROSY (measures $\mathrm{N}-\mathrm{C}_{\alpha}$ couplings) experiments. Under the same conditions, the spin-state-selection approaches appear to be more precise. It is also noted that when the ${ }^{1} \mathrm{~J}_{\mathrm{N}-\mathrm{C} \alpha}$ couplings are small, their values measured from the $\mathrm{HN}\left(\mathrm{CO}-\alpha / \beta-\mathrm{NC}_{\alpha}-\mathrm{J}\right)$-TROSY experiment tend to be systematically smaller than those measured from a quantitative J-correlated $\left[{ }^{15} \mathrm{~N},{ }^{1} \mathrm{H}\right]$-TROSY-HNC experiment. A possible explanation is that the covariance effects of in-phase doublets from the $\mathrm{HN}(\mathrm{CO}-\alpha / \beta-$ $\mathrm{NC}_{\alpha}$-J)-TROSY experiment cause an underestimation of the ${ }^{1} \mathrm{~J}_{\mathrm{N}-\mathrm{C} \alpha}$ couplings, but no such effect exists for ${ }^{2} \mathrm{~J}_{\mathrm{N}-\mathrm{C} \alpha}$ because they are measured from two separated spectra. The correlation coefficients for ${ }^{1} \mathrm{D}_{\mathrm{N}-\mathrm{C}^{\prime}}$, ${ }^{1} \mathrm{D}_{\mathrm{N}-\mathrm{C} \alpha}$ and ${ }^{2} \mathrm{D}_{\mathrm{N}-\mathrm{C} \alpha}$ couplings measured from the two different approaches are, respectively, $0.976,0.922$ and 0.293 . The low measurement precision in both approaches is thought to be responsible for the poor correlation of ${ }^{2} \mathrm{D}_{\mathrm{N}-\mathrm{C} \alpha}$ couplings. Thus, caution must be taken when ${ }^{2} \mathrm{D}_{\mathrm{N}-\mathrm{C} \alpha}$ RDCs are used in either structural or dynamical study if the uncertainty of measurement is comparable with the magnitude of the ${ }^{2} \mathrm{D}_{\mathrm{N}-\mathrm{C} \alpha}$ RDCs.

A 3D HNCA( $\alpha / \beta$-J-CAHAN)-TROSY experiment has been designed to measure ${ }^{1} \mathrm{~J}_{\mathrm{C}_{\alpha} \mathrm{H}_{\alpha}},{ }^{2} \mathrm{~J}_{\mathrm{N}-\mathrm{H} \alpha}$ and ${ }^{3} \mathrm{~J}_{\mathrm{N}-\mathrm{H} \alpha}$ couplings [124]. The coherence transfer scheme is similar to an earlier HNCA-TROSY experiment [92]:

$$
{ }^{1} \mathrm{H}_{\mathrm{N}} \rightarrow{ }^{15} \mathrm{~N} \rightarrow{ }^{13} \mathrm{C}_{\alpha}\left(\mathrm{t}_{1}\right) \rightarrow{ }^{15} \mathrm{~N}\left(\mathrm{t}_{2}\right) \rightarrow{ }^{1} \mathrm{H}_{\mathrm{N}}\left(\mathrm{t}_{3}\right)
$$

After the magnetization is transferred to $\mathrm{C}_{\alpha}$ from ${ }^{15} \mathrm{~N}$, a spin-state-filter is used so that either an $8 \mathrm{H}_{\mathrm{N}}^{\mathrm{z}} \mathrm{N}^{\mathrm{z}} \mathrm{H}_{\alpha}^{\mathrm{z}} \mathrm{C}_{\alpha}^{\mathrm{y}}$ or $4 \mathrm{H}_{\mathrm{N}}^{\mathrm{z}} \mathrm{N}^{\mathrm{z}} \mathrm{C}_{\alpha}^{\mathrm{y}}$ term evolves during the $t_{1}$ period, yielding, respectively, the anti-phase and in-phase doublets of ${ }^{13} \mathrm{C}_{\alpha}-\left\{{ }^{1} \mathrm{H}_{\alpha}\right\}$ couplings. The ${ }^{1} \mathrm{H}_{\alpha}$ is coupled, respectively, to ${ }^{13} \mathrm{C}$ (during the $t_{1}$ period) and ${ }^{15} \mathrm{~N}$ (during the $t_{2}$ period). The cross peaks in a plane $\left[{ }^{13} \mathrm{C},{ }^{15} \mathrm{~N}\right]$ appear as an E.COSY pattern since the spin states of ${ }^{1} \mathrm{H}_{\alpha}$ are not mixed. Two sub-spectra are generated after the addition and subtraction of the in-phase and anti-phase 
experiments. The resonances associated with the intra $\mathrm{C}_{\alpha}$ peaks appeared in $\left[{ }^{13} \mathrm{C},{ }^{15} \mathrm{~N}\right]$ planes at, respectively, $\left\{\omega_{\mathrm{C} \alpha(\mathrm{i})}+\pi^{1} \mathrm{~J}_{\mathrm{C} \alpha \mathrm{H}_{\alpha}}, \omega_{\mathrm{N}(\mathrm{i})}-\pi^{2} \mathrm{~J}_{\mathrm{NH} \alpha}\right\}$ and $\left\{\omega_{\mathrm{C} \alpha(\mathrm{i})}-\pi^{1} \mathrm{~J}_{\mathrm{C} \alpha \mathrm{H}_{\alpha}}, \omega_{\mathrm{N}(\mathrm{i})}+\pi^{2} \mathrm{~J}_{\mathrm{NH} \alpha}\right\}$. However, the shifts due to the TROSY scheme are not shown since they are irrelevant for coupling determination. The resonances associated with the inter $\mathrm{C}_{\alpha}$ peaks can also be obtained and the couplings of ${ }^{1} \mathrm{~J}_{\mathrm{C}_{\alpha} \mathrm{H}_{\alpha}},{ }^{2} \mathrm{~J}_{\mathrm{N}-\mathrm{H} \alpha}$ and ${ }^{3} \mathrm{~J}_{\mathrm{N}-\mathrm{H} \alpha}$ can be deduced readily. Two other experiments to measure the same couplings have also been proposed in the same paper [124]. The experiments have been tested on $1.9 \mathrm{mM}{ }^{13} \mathrm{C} /{ }^{15} \mathrm{~N}$-labeled ubiquitin (76 residues). A detailed discussion was given as to how the accuracy for the measurement of $\mathrm{C}_{\alpha}-\mathrm{H}_{\alpha}$ couplings is affected by the DD-DD cross-correlation between $\mathrm{C}_{\alpha}-\mathrm{H}_{\alpha}$ dipolar and ${ }^{1} \mathrm{H}_{\alpha}-\mathrm{H}_{\mathrm{r}}$ dipolar interactions $\left(\mathrm{H}_{\mathrm{r}}\right.$ is a remote proton coupled to ${ }^{1} \mathrm{H}_{\alpha}$ ), and by the homonuclear $\mathrm{J}_{\mathrm{C}_{\alpha} \mathrm{C}_{\beta}}$ coupling during the $t_{1}$ period. The authors also discussed the errors in the measurement of ${ }^{2} \mathrm{~J}_{\mathrm{N}-\mathrm{H} \alpha}$ and ${ }^{3} \mathrm{~J}_{\mathrm{N}-\mathrm{H} \alpha}$ couplings caused by the partial collapse of E.COSY patterns, which in turn, is caused by the ${ }^{1} \mathrm{H}$ spin flip between the $t_{1}$ and $t_{2}$ evolution periods.

A 2D- $\left[{ }^{13} \mathrm{C},{ }^{13} \mathrm{C}\right]$-TOCSY experiment has been developed to measure side-chain ${ }^{13} \mathrm{C}-{ }^{13} \mathrm{C}$ RDCs $[125$, 126]. Side-chain ${ }^{13} \mathrm{C}-{ }^{13} \mathrm{C}$ RDCs may prove to be indispensable for high-quality structure determinations of perdeuterated proteins, since for such protein samples there are, in general, not enough NOE restraints to define side-chain conformations. The proposed experiment starts from ${ }^{13} \mathrm{C}$, after the chemical shift labeling of ${ }^{13} \mathrm{C}$, the magnetization is transferred to other ${ }^{13} \mathrm{C}$ nuclei using a FLOPSY-16 scheme. The couplings between aliphatic ${ }^{13} \mathrm{C}$ nuclei are active during the direct ${ }^{13} \mathrm{C}$ observation period. Thus, the cross peaks along the direct ${ }^{13} \mathrm{C}$ dimension split into doublets, quadruplets, and octets dependent upon the numbers of ${ }^{13} \mathrm{C}$ nuclei directly attached to the observed ${ }^{13} \mathrm{C}$. The couplings can be readily available from the doublets. Theoretically, the sum of two RDC values from the outer components in the quadruplet can also be decomposed into individual contributions by starting from the outermost ${ }^{13} \mathrm{C}$ nuclei. The sum can also be used directly as structural constraints [114]. When applied on ${ }^{13} \mathrm{C} /{ }^{15} \mathrm{~N} /{ }^{2} \mathrm{H}$-labeled ubiquitin, $23{ }^{13} \mathrm{C}-{ }^{13} \mathrm{C}$ RDCs can be extracted from the doublets, and 10 RDCs can be obtained from the outer components of quadruplets. The deviation between the RDCs measured independently from different cross peaks is less than $0.3 \mathrm{~Hz}$.

A 3D HCACO $\left(\alpha / \beta-\mathrm{C}^{\prime} \mathrm{HA}\right)$ experiment has been designed to measure ${ }^{2} \mathrm{~J}_{\mathrm{C}^{\prime}-\mathrm{H} \alpha}$ and ${ }^{1} \mathrm{~J}_{\mathrm{C} \alpha-\mathrm{H} \alpha}$ couplings [87]. The experiment is suitable for proline-rich proteins or for proteins which must be studied at high $\mathrm{pH}$ or temperature conditions. The magnetization transfer pathway is as follows:

$$
{ }^{1} \mathrm{H}_{\alpha} \rightarrow{ }^{13} \mathrm{C}_{\alpha} \rightarrow{ }^{13} \mathrm{C}^{\prime}\left(\mathrm{t}_{1}\right) \rightarrow{ }^{13} \mathrm{C}_{\alpha}\left(\mathrm{CT}, \mathrm{t}_{2}\right) \rightarrow{ }^{1} \mathrm{H}_{\alpha}\left(\mathrm{t}_{3}\right)
$$

A spin-state-filter (using $\mathrm{J}_{\mathrm{H} \alpha-\mathrm{C} \alpha}$ ) is inserted in the INEPT magnetization transfer step (from ${ }^{13} \mathrm{C}_{\alpha}$ to $\mathrm{C}^{\prime}$ ) to generate either a $2 \mathrm{C}_{\alpha}^{\mathrm{z}} \mathrm{C}^{\prime \mathrm{y}}$ or $4 \mathrm{H}_{\alpha}^{\mathrm{z}} \mathrm{C}_{\alpha}^{\mathrm{z}} \mathrm{C}^{\prime y}$ term which evolves into observable terms after the $t_{1}$ period. Both the $\mathrm{C}^{\prime}$ chemical shifts and the couplings between $\mathrm{C}^{\prime}$ and ${ }^{1} \mathrm{H}_{\alpha}$ evolve during the $t_{1}$ period, while the ${ }^{13} \mathrm{C}$ chemical shifts and the couplings between $\mathrm{C}_{\alpha^{-}}{ }^{1} \mathrm{H}_{\alpha}$ evolve during the $t_{2}$ period. Since the spin states of ${ }^{1} \mathrm{H}_{\alpha}$ are not mixed between the $t_{1}$ and $t_{2}$ periods, cross peaks with E.COSY patterns appear in the $\left[\mathrm{C}^{\prime}, \mathrm{C}_{\alpha}\right]$ correlation planes. After the addition and subtraction of the in-phase and anti-phase spectra, the two cross peaks in an E.COSY pattern are further separated into two sub-spectra according to the upfield or downfield component of a $\mathrm{C}^{\prime}$ spin. Thus, the small splitting of ${ }^{2} \mathrm{~J}_{\mathrm{C}^{\prime}-\mathrm{H} \alpha}$ becomes well-resolved indirectly, and can be measured accurately. To increase the measurement accuracy, a large number of time points are required for both the $t_{1}$ and $t_{2}$ dimensions. The pulse scheme has been demonstrated on ${ }^{13} \mathrm{C} /{ }^{15} \mathrm{~N}$-labeled ubiquitin samples under both the isotropic and anisotropic conditions.

A 3D TROSY-HNCO based-experiment has been developed to measure the $\mathrm{N}-\mathrm{C}^{\prime}$ and $\mathrm{N}-\mathrm{H}$ couplings [109] using a total of three experiments with one of them to be a common reference experiment. For the measurement of N-C' couplings, it uses the same pulse sequence scheme as in a 3D TROSY-HNCO quantitative $\mathrm{J}_{\mathrm{N}-\mathrm{C}^{\prime}}$ experiment [99] (Section 3.5). Two experiments are used in that approach with the peak intensity being modu- 
lated by $\mathrm{J}_{\mathrm{N}-\mathrm{C}^{\prime}}$ during the $\mathrm{C}^{\prime}$ evolution period. The experiment with the optimal intensity is called a reference experiment. In order to use the reference experiment for measuring the H-N couplings, Vijayan and Zweckstetter added a third TROSY-based HNCO experiment, where an amplified H-N coupling evolution period is inserted right after the constant-time ${ }^{15} \mathrm{~N}$ evolution period. The cross peaks appear at $\omega_{\mathrm{N}}-(\kappa+1) \pi^{1} \mathrm{~J}_{\mathrm{NH}}$ along the ${ }^{15} \mathrm{~N}$ dimension in the third experiment, while in the reference spectrum they appear at $\omega_{\mathrm{N}}-\pi^{1} \mathrm{~J}_{\mathrm{NH}}$. The couplings can be extracted readily from these two experiments. By sharing the same reference experiment and acquiring the three experiments in an interleaved manner, the experiment can save up to $25 \%$ instrumental time. The experiment has been demonstrated on both ${ }^{13} \mathrm{C} /{ }^{15} \mathrm{~N}$-labeled $0.8 \mathrm{mM}$ ubiquitin and $0.8 \mathrm{mM}$ DcuS $(17 \mathrm{kDa})$. The experiment is applicable for proteins which can yield a TROSY-HNCO spectrum with S/N > 20:1 in a reasonable amount of time.

A $3 \mathrm{D} \mathrm{CBCA}(\mathrm{CO}) \mathrm{NH}$ experiment has been developed to measure the one-bond couplings of ${ }^{13} \mathrm{C}_{\alpha^{-}}{ }^{1} \mathrm{H}_{\alpha}$, ${ }^{13} \mathrm{C}_{\beta^{-}}{ }^{1} \mathrm{H}_{\beta}$ and ${ }^{13} \mathrm{C}_{-}{ }^{13} \mathrm{C}^{\prime}$ [109] where four spectra are acquired in an interleaved manner. The ${ }^{13} \mathrm{C}_{\alpha^{-}}{ }^{1} \mathrm{H}_{\alpha}$ and ${ }^{13} \mathrm{C}_{\beta^{-}}{ }^{1} \mathrm{H}_{\beta}$ couplings are measured in a same manner as discussed earlier in this section [120]. The fourth experiment is the same as a regular $\mathrm{CBCA}(\mathrm{CO}) \mathrm{NH}$ (reference) experiment used to measure the couplings of ${ }^{13} \mathrm{C}_{\alpha^{-}}{ }^{1} \mathrm{H}_{\alpha}$ and ${ }^{13} \mathrm{C}_{\beta^{-}}{ }^{1} \mathrm{H}_{\beta}$, except that $\mathrm{J}_{\mathrm{C}^{\prime}-\mathrm{C} \alpha}$ is active in the step of $\mathrm{C}^{\prime} \rightarrow{ }^{15} \mathrm{~N}$ transfer for about $18.7 \mathrm{~ms}$, not 9 $\mathrm{ms}$ as in the reference experiment. The $\mathrm{C}_{\alpha}-\mathrm{C}^{\prime}$ couplings can be extracted from the intensity ratio of the fourth and the reference experiments. Compared to the measurement of $\mathrm{C}_{\alpha}-\mathrm{C}^{\prime}$ couplings using an HNCO-based quantitative-J modulation experiment [80], the resolution of $\mathrm{CBCA}(\mathrm{CO}) \mathrm{NH}$ is usually lower. However, the low resolution is offset somewhat by the possibility that the $\mathrm{C}_{\alpha}-\mathrm{C}^{\prime}$ couplings can be extracted from both $\mathrm{C}_{\alpha}$ and $\mathrm{C}_{\beta}$ resonances. Analysis showed that for an $\mathrm{S} / \mathrm{N}$ ratio of 50:1 in the reference spectrum, a random error of $0.35 \mathrm{~Hz}$ is observed in $\mathrm{J}_{\mathrm{C} \alpha-\mathrm{C}^{\prime}}$ coupling measurement. The experiments have been demonstrated on both ${ }^{13} \mathrm{C} /{ }^{15} \mathrm{~N}$-labeled $0.8 \mathrm{mM}$ ubiquitin and $0.8 \mathrm{mM}$ DcuS $(17 \mathrm{kDa})$.

A 3D CH $\mathrm{CH}_{2}-\mathrm{S}^{3} \mathrm{CT} \mathrm{HBCBCA}$ experiment has been designed to measure eight couplings for $\mathrm{CH}_{2}-\mathrm{CH}$ moiety in proteins and nucleic acids [127]. The pulse scheme is an extension of a 2D $\mathrm{CH}_{2}$-TROSY experiment [128]. It is an out-and-back type experiment where the magnetization starts from and ends at two ${ }^{1} \mathrm{H}_{\beta}$ protons. For simplicity, only one ${ }^{1} \mathrm{H}_{\beta}$ is shown in the following magnetization transfer pathway:

$$
{ }^{1} \mathrm{H}_{\beta 2} \rightarrow{ }^{13} \mathrm{C}_{\beta} \rightarrow{ }^{13} \mathrm{C}_{\alpha}\left(\mathrm{t}_{1}\right) \rightarrow{ }^{13} \mathrm{C}_{\beta}\left(\mathrm{CT}, \mathrm{t}_{2}\right) \rightarrow{ }^{1} \mathrm{H}_{\beta 2}\left(\mathrm{t}_{3}\right)
$$

During the $\mathrm{C}_{\alpha}$ chemical shift evolution period $\left(t_{1}\right)$, the couplings between $\mathrm{C}_{\alpha}$ and the three protons $\left({ }^{1} \mathrm{H}_{\alpha}\right.$ and two ${ }^{1} \mathrm{H}_{\beta}$ ) are active, while the couplings between $\mathrm{C}_{\alpha}$ and $\mathrm{C}_{\beta}, \mathrm{C}^{\prime}$ and ${ }^{15} \mathrm{~N}$ are refocused to increase the sensitivity. Similarly, during the $\mathrm{C}_{\beta}$ chemical shift evolution $\left(t_{2}\right)$ period, the couplings between $\mathrm{C}_{\beta}$ and the three protons $\left({ }^{1} \mathrm{H}_{\alpha}\right.$ and two $\left.{ }^{1} \mathrm{H}_{\beta}\right)$ are active. There are no ${ }^{1} \mathrm{H}$ pulses between $t_{1}$ and $t_{2}$ periods, and thus the spin states of ${ }^{1} \mathrm{H}_{\alpha}, \mathrm{H}_{\beta 2}$ and $\mathrm{H}_{\beta 3}$ are preserved and each observable term has specific transition pathway. In the final stage, a $\mathrm{CH}_{2}$ spin-state-selective scheme is used to transfer the magnetization from $\mathrm{C}_{\beta}$ to its attached two ${ }^{1} \mathrm{H}_{\beta}$ protons. The ${ }^{1} \mathrm{H}_{\alpha}$ spin experiences a net $360^{\circ}$ rotation in the last steps since no pulses are applied on $\mathrm{C}_{\alpha}$ in the last transition step. Finally, four components are observed for each cross peak as in a regular 3D HBCBCA experiment. Conceptually, eight couplings of ${ }^{1} \mathrm{~J}_{\mathrm{C} \alpha \mathrm{H} \alpha},{ }^{2} \mathrm{~J}_{\mathrm{C} \alpha \mathrm{H} \beta 2}+{ }^{2} \mathrm{~J}_{\mathrm{C} \alpha \mathrm{H} \beta 3},{ }^{2} \mathrm{~J}_{\mathrm{C} \beta \mathrm{H} \alpha},{ }^{1} \mathrm{~J}_{\mathrm{C} \beta \mathrm{H} \beta 2}+{ }^{1} \mathrm{~J}_{\mathrm{C} \beta \mathrm{H} \beta 3}$, ${ }^{1} \mathrm{~J}_{\mathrm{C} \beta \mathrm{H} \beta 2}-{ }^{2} \mathrm{~J}_{\mathrm{H} \beta 2 \mathrm{H} \beta 3},{ }^{1} \mathrm{~J}_{\mathrm{C} \beta \mathrm{H} \beta 3}-{ }^{2} \mathrm{~J}_{\mathrm{H} \beta 2 \mathrm{H} \beta 3},{ }^{3} \mathrm{~J}_{\mathrm{H} \alpha \mathrm{H} \beta 2}$ and ${ }^{3} \mathrm{~J}_{\mathrm{H} \alpha \mathrm{H} \beta 3}$ can be extracted from the frequency displacement of the corresponding two components in the 3D spectra. Interested readers can refer to the original work for the details on how to extract eight couplings from four components. Although both the precision of measurement and sensitivity are a concern since so many active couplings are allowed in the experiment, several nice features make the experiment a rather sensitive one. These features include (a) all the four observed components are relaxation favorable components; (b) significant linewidth broadening from small couplings such as ${ }^{2} \mathrm{~J}_{\mathrm{C} \alpha \mathrm{H} \beta 2},{ }^{2} \mathrm{~J}_{\mathrm{C} \beta \mathrm{H} \alpha},{ }^{3} \mathrm{~J}_{\mathrm{H} \alpha \mathrm{H} \beta 2}$ and ${ }^{3} \mathrm{~J}_{\mathrm{H} \alpha \mathrm{H} \beta 3}$ is removed; (c) finally, each coupling can be measured independently two to four times, improving the measurement precision. When tested on a $1.5 \mathrm{mM}{ }^{13} \mathrm{C} /{ }^{15} \mathrm{~N}$ - 

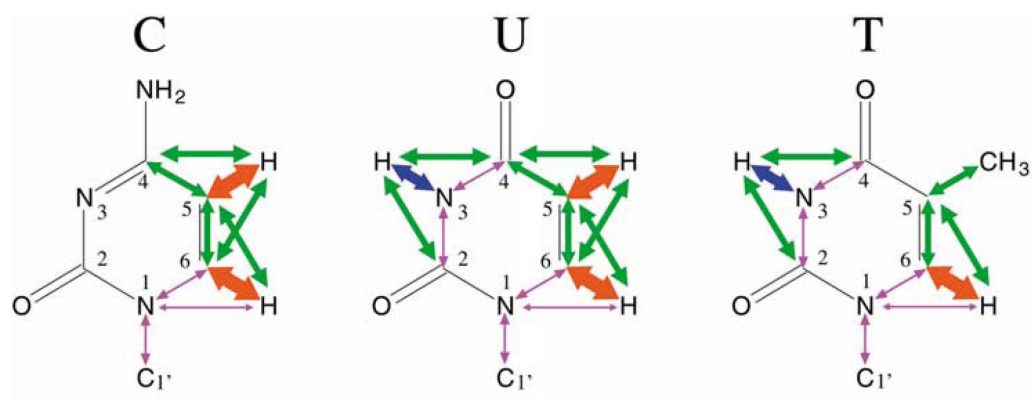

A
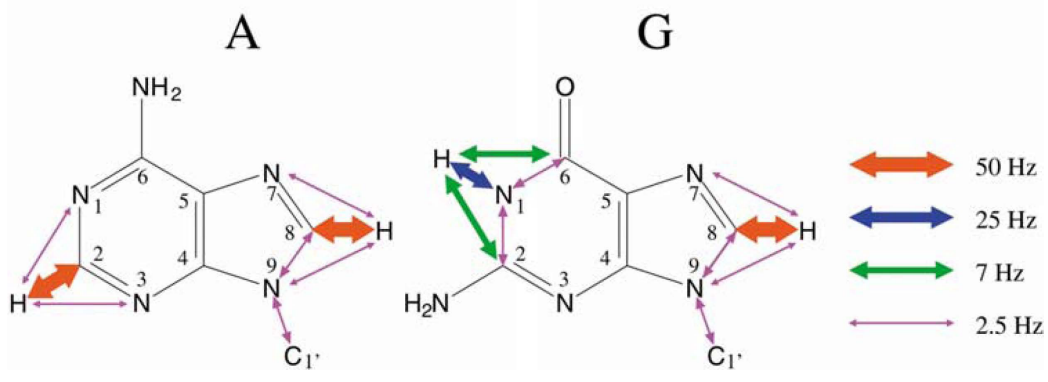

Figure 9: Measurable residual dipolar couplings in nucleic acid bases. The line thickness represents the upper limit of RDC magnitudes. The scale on right is calibrated to a maximal ${ }^{1} \mathrm{D}_{\mathrm{NH}}$ coupling of $25 \mathrm{~Hz}$. Reprinted from [129], with permission from Springer Science and Business Media.

labeled third IgG-binding domain from Streptococcal Protein G (GB3), it was found the error was well below $0.5 \mathrm{~Hz}$ for the majority of the couplings. The application of the experiment is limited to certain residues with relatively-isolated $\mathrm{CH}_{2}-\mathrm{CH}$ moiety such as $\mathrm{C}, \mathrm{F}, \mathrm{Y}, \mathrm{W}, \mathrm{D}, \mathrm{N}$ and $\mathrm{H}$. Other residues don't interfere with the extraction of couplings from these residues since either the signals from the former are suppressed by a factor of 10 or larger, or the ${ }^{1} \mathrm{H}_{\beta}$ chemical shifts of the former are in regions different from the residues with relatively-isolated $\mathrm{CH}_{2}-\mathrm{CH}$ moiety.

\section{Measurement of residual dipolar couplings in nucleic acids}

Traditional NMR methods [74] for structure determination of nucleic acids rely mainly on distance restraints from NOE and dihedral angle restraints from scalar coupling. However, due to the low proton density, the amount of restraints measurable by the traditional NMR methods is not sufficient for computing precise and accurate structures. In addition, the elongated shape of nucleic acids makes it challenging to compute precise structures. The newly-introduced RDC constraints provide much needed additional global restraints, since with RDCs the orientations of all the internuclear vectors in a nucleic acid can be evaluated with respect to a unique global frame. Most current experiments for measuring RDCs in nucleic acid bases use TROSY and multiple-quantum (MQ) coherence schemes to minimize the loss of coherence by relaxation. Furthermore, E.COSY scheme is often employed to overcome peak overlap problems, especially for measuring RDCs in ribose. Compared to amino acids, the structural networks in nucleic bases are more complicated and the chemical shift ranges of both ${ }^{13} \mathrm{C}$ and ${ }^{15} \mathrm{~N}$ are much wider. Therefore, shaped pulses are often used in the experiments. Shown in Fig. 9 are some measurable RDCs in nucleic acid bases [129]. 


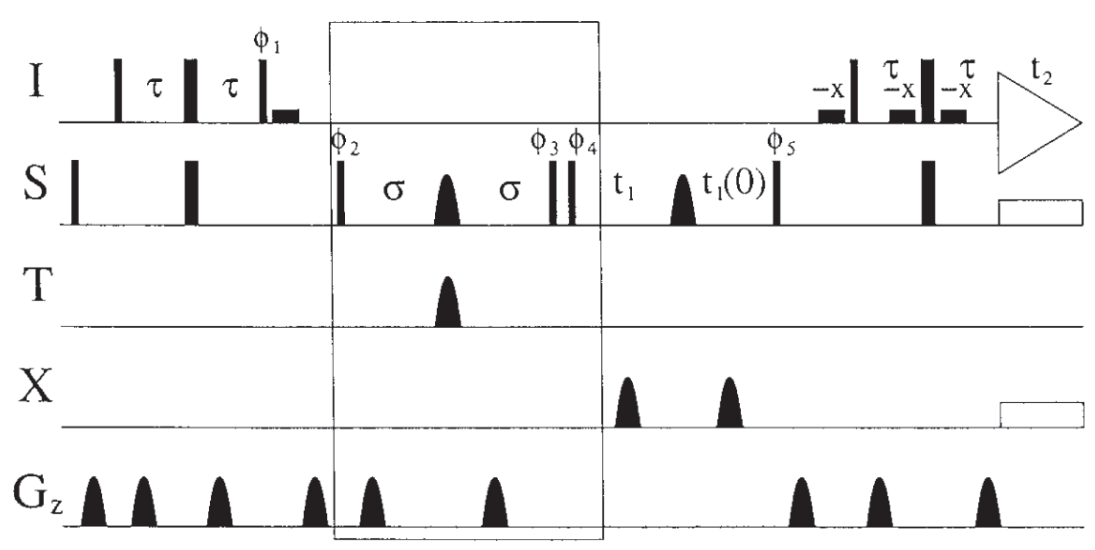

Figure 10: A schematic representation of a basic $\mathbf{S}^{3} \mathbf{E} \mathbf{I S}[\mathbf{T}]$ experiment. Narrow and wide bars indicate hard $90^{\circ}$ and $180^{\circ}$ pulses, respectively. Shown in the box is the $\mathrm{S}^{3} \mathrm{E}$ element. The experiment can be used to measure the couplings of both S-T and I-T. More technical details can be found in the original paper. Reprinted from [129], with permission from Springer Science and Business Media.

\subsection{RDC measurement in nucleic acid bases}

A suite of spin-state-selective excitation $\left(\mathrm{S}^{3} \mathrm{E}\right)$-IS(T) experiments have been developed to measure many one and two-bond couplings in purine and pyrimidine bases [129]. Showed in Fig. 10 is a basic experiment typical for measuring coupling in nucleic acids. The I spin represents ${ }^{1} \mathrm{H}, \mathrm{S}$ represents either ${ }^{13} \mathrm{C}$ or ${ }^{15} \mathrm{~N}$ dependent on which couplings are being measured. $\mathrm{T}$ is coupled to $\mathrm{S}$, and $\mathrm{X}$ is decoupled from $\mathrm{S}$ during the chemical shift evolution of $S$ spin. The unit in the rectangular box is an $S^{3} E$ scheme $[39,40]$, which can separate $S_{x} T^{\alpha}$ and $\mathrm{S}_{x} \mathrm{~T}^{\beta}$ states into two sub-spectra. Furthermore, if nucleus $\mathrm{T}$ is coupled during both $t_{1}$ evolution and acquisition periods, the components of doublet in two sub-spectra shift in both $\mathrm{S}$ and I dimensions away from their respective resonance frequencies by the values of $\pm \mathrm{J}_{\mathrm{S}-\mathrm{T}} / 2$ and $\pm \mathrm{J}_{\mathrm{I}-\mathrm{T}} / 2$. When these two spectra are added, the doublet appear as typical E.COSY pattern. Different pulse sequences can be derived from this basic scheme by tuning the shaped pulses to measure many different one and two-bond RDCs as shown in Fig. 9. For example, HC[N] experiment yields the values of ${ }^{1} \mathrm{D}\left(\mathrm{N} 1 \mathrm{C} 1{ }^{\prime}\right),{ }^{1} \mathrm{D}(\mathrm{N} 1 \mathrm{C} 6)$ and ${ }^{2} \mathrm{D}(\mathrm{N} 1 \mathrm{H} 6)$ in pyrimidines, and ${ }^{1} \mathrm{D}$ (N9C1'), ${ }^{1} \mathrm{D}(\mathrm{N} 9 \mathrm{C} 8)$ and ${ }^{2} \mathrm{D}(\mathrm{N} 9 \mathrm{H} 8)$ in purines. HN[C] experiment measures ${ }^{1} \mathrm{D}(\mathrm{NC})$ and ${ }^{2} \mathrm{D}\left(\mathrm{C}-\mathrm{H}_{N}\right)$ couplings in $\mathrm{H}-\mathrm{N}-\mathrm{C}$ moieties of thymines, uracils, and guanines. $\mathrm{HC}[\mathrm{C}]$ experiment determines ${ }^{1} \mathrm{D}(\mathrm{CC})$ and ${ }^{2} \mathrm{D}(\mathrm{CH})$ couplings at position 5 and 6 in pyrimidines. $\mathrm{HN}[\mathrm{H}]$ experiment yields the couplings of ${ }^{2} \mathrm{D}(\mathrm{N} 1 \mathrm{H} 2)$, ${ }^{2} \mathrm{D}(\mathrm{N} 3 \mathrm{H} 2),{ }^{2} \mathrm{D}(\mathrm{N} 7 \mathrm{H} 8)$, and ${ }^{2} \mathrm{D}(\mathrm{N} 9 \mathrm{H} 8)$ in purines. In the last experiment, the magnetization of the proton in moiety of H-C-N is transferred directly to the ${ }^{15} \mathrm{~N}$ through two-bond scalar couplings because the one-bond ${ }^{1} \mathrm{~J}_{\mathrm{NC}}$ is too small. These experiments have been tested on a ${ }^{13} \mathrm{C} /{ }^{15} \mathrm{~N}$-labeled DNA hairpin and a 12-mer DNA duplex sample. The accuracy and precision of the RDC measurement have been discussed in great details in the original paper [129].

MQ-HCN experiment plays a key role in correlating the ribose and base through the $\mathrm{N}_{1}$ or $\mathrm{N}_{9}$ nucleus [130]. HCN experiments have been reported from several groups. Here we only review the more recent ones $[131,132,62,133]$. The experiments use an out-and-back scheme and either correlate the three nuclei of moiety $\mathrm{H}_{1^{\prime} / 6 / 8}-\mathrm{C}_{1^{\prime} / 6 / 8}-\mathrm{N}_{1 / 9}$, or directly correlate $\mathrm{H}_{1^{\prime}}$ with base $\mathrm{H}_{6 / 8}$ using an $\mathrm{H}_{\mathrm{s}} \mathrm{C}_{\mathrm{s}} \mathrm{N}_{\mathrm{b}} \mathrm{C}_{\mathrm{b}} \mathrm{H}_{\mathrm{b}}$ scheme through the detection of three frequencies from five different nuclei, where $s$ and $b$ indicate, respectively, a ribose and base. Another interesting experiment is the bi-directional 3D MQ-HCNCH, which dictates all the five frequencies in the moiety of $\mathrm{H}_{\mathrm{s}} \mathrm{C}_{\mathrm{s}} \mathrm{N}_{\mathrm{b}} \mathrm{C}_{\mathrm{b}} \mathrm{H}_{\mathrm{b}}$, and is a powerful tool for correlating bases and riboses [134]. MQ-HCN experiment offers excellent resolutions and is very useful for the measurement of many different 
types of RDCs in nucleic acids. The RDCs of $\mathrm{C}_{1^{\prime}}-\mathrm{H}_{1^{\prime}}, \mathrm{C}_{6 / 8}-\mathrm{H}_{6 / 8}$ can be measured with a 3D MQ-HCN if the ${ }^{13} \mathrm{C}$ is not decoupled during the data acquisition. The 3D HCN experiment [130] with intrinsic high resolution is also a favorable choice for measuring large $\mathrm{C}-\mathrm{H}$ couplings since there is no significant difference in relaxation rates between the different components of ${ }^{1} \mathrm{H}$ doublets. To measure the one-bond couplings of $\mathrm{C}_{1^{\prime}}-\mathrm{N}_{1 / 9}$ and $\mathrm{C}_{6 / 8}-\mathrm{N}_{1 / 9}$ using the MQ-HCN experiment, a $\mathrm{J}_{\mathrm{CN}}$ enhance factor (about 5-fold amplification) is used in the ${ }^{13} \mathrm{C}$ chemical shift evolution. Since the spin states of ${ }^{15} \mathrm{~N}$ are not perturbed after the $t_{2}$ period and no ${ }^{15} \mathrm{~N}$ decoupling is applied during the data acquisition, the couplings of ${ }^{15} \mathrm{~N}$ to both ${ }^{13} \mathrm{C}$ and proton make a doublet appear as an E.COSY pattern, and thus ${ }^{2} \mathrm{~J}_{\mathrm{HN}}$ can be readily extracted. This E.COSY-based MQ-HCN sequence can be used to determine the couplings of $\mathrm{C}_{1^{\prime}}-\mathrm{N}_{1 / 9}, \mathrm{C}_{6}-\mathrm{N}_{1}, \mathrm{C}_{8}-\mathrm{N}_{9}, \mathrm{H}_{1^{\prime}}-\mathrm{N}_{1 / 9}, \mathrm{H}_{6}-\mathrm{N}_{1}$ and $\mathrm{H}_{8}-\mathrm{N}_{9}$. Similarly, if the one-bond ${ }^{13} \mathrm{C}_{-}{ }^{13} \mathrm{C}$ coupling is active during either the $\mathrm{C}_{1^{\prime}}$ or $\mathrm{C}_{6}$ evolution period, the MQ-HCN experiment can measure the couplings of $\mathrm{C}_{1^{\prime}}-\mathrm{C}_{2^{\prime}}$ and $\mathrm{C}_{6}-\mathrm{C}_{5}$. If only $\mathrm{C}_{1^{\prime}}$ and $\mathrm{C}_{6}$ are selectively decoupled, the two bond couplings of $\mathrm{H}_{1^{\prime}}-\mathrm{C}_{2^{\prime}}$ and $\mathrm{H}_{6}-\mathrm{C}_{5}$ can also be detected in an E.COSY pattern. All the experiments have been demonstrated on a $0.4 \mathrm{mM}{ }^{13} \mathrm{C} /{ }^{15} \mathrm{~N}$-labeled 18 bp DNA duplex in a $47 \mathrm{kDa}$ ternary complexed with two proteins, $\mathrm{CBF}_{\beta}$ and $\mathrm{CBF}_{\alpha}$. The accuracy of measurement has been discussed and the RMSD from two independent measurements have been listed for RDCs of different spin pairs.

$\mathrm{HC}(\mathrm{C})$ hd-TROSY-E. COSY experiment measures ${ }^{1} \mathrm{D}_{\mathrm{CC}}$ and ${ }^{2} \mathrm{D}_{\mathrm{CH}}$ couplings in pyrimidines (U and C) [135], where "hd" stands for proton homo-decoupling. It is a pseudo $3 \mathrm{D}$ experiment where the ${ }^{1} \mathrm{H}$ and ${ }^{13} \mathrm{C}$ chemical shifts are labeled by using either a $\mathrm{C}_{5}\left(t_{1}\right)-\mathrm{H}_{5}\left(t_{2}\right)-\mathrm{H}_{5}\left(\mathrm{t}_{3}\right)$ or $\mathrm{C}_{6}\left(t_{1}\right)-\mathrm{H}_{6}\left(t_{2}\right)-\mathrm{H}_{6}\left(\mathrm{t}_{3}\right)$ scheme. During the $\mathrm{C}_{5}\left(t_{1}\right)$ evolution, either the ${ }^{1} \mathrm{~J}_{\mathrm{CC}}$ coupling between $\mathrm{C}_{5}$ and $\mathrm{C}_{4}$ or that between $\mathrm{C}_{5}$ and $\mathrm{C}_{6}$ evolves selectively. During the $\mathrm{H}_{5}\left(t_{2}\right)$ evolution, a proton homo-decoupling is used to suppress unwanted couplings. A very narrow spectral window is used in the $t_{2}$ dimension and the resulting aliasing is unfolded with the $t_{3}$ dimension to get a $2 \mathrm{D}\left[{ }^{1} \mathrm{H},{ }^{13} \mathrm{C}\right]$ correlated spectrum with a proton-decoupled ${ }^{1} \mathrm{H}$ dimension [66]. During the direct detection, no ${ }^{13} \mathrm{C}$ decoupling is applied. Since the $\alpha$ and $\beta$ states of $\mathrm{C}_{4}$ or $\mathrm{C}_{6}$ are not mixed during the experiment, an E.COSY pattern appears which allows the couplings $\mathrm{H}_{5}-\mathrm{C}_{4}$ or $\mathrm{H}_{5}-\mathrm{C}_{6}$ be measured readily from the peak displacement along the ${ }^{1} \mathrm{H}$ dimension. The experiments can determine the couplings of ${ }^{2} \mathrm{D}_{\mathrm{C} 5-\mathrm{H} 6}$, ${ }^{2} \mathrm{D}_{\mathrm{C} 6-\mathrm{H} 5},{ }^{2} \mathrm{D}_{\mathrm{C} 4-\mathrm{H} 5},{ }^{1} \mathrm{D}_{\mathrm{C} 5-\mathrm{C} 6}$ and ${ }^{1} \mathrm{D}_{\mathrm{C} 4-\mathrm{C} 5}$. One bond couplings $\left(\mathrm{C}_{5}-\mathrm{H}_{5}\right.$ and $\left.\mathrm{C}_{6}-\mathrm{H}_{6}\right)$ can also be measured with the sequence hd-TROSY. To measure the couplings of $\mathrm{C}_{4}-\mathrm{C}_{5}$ or $\mathrm{C}_{5}-\mathrm{C}_{6}$ in purines, an experiment called $\mathrm{HCC}(\mathrm{C})-\mathrm{TOCSY}$ has been proposed. $\mathrm{HCC}(\mathrm{C})$ stands for $\mathrm{H} 8 \mathrm{C} 8 \mathrm{C} 4(\mathrm{C} 5)$ or $\mathrm{H} 8 \mathrm{C} 8 \mathrm{C} 6(\mathrm{C} 5)$ for guanine. Thus, the 3D experiment uses an out-and-back scheme with magnetization started from $\mathrm{H}_{8}$, and then selectively transferred to either $\mathrm{C}_{4}$ or $\mathrm{C}_{6}$ through $\mathrm{C}_{8}$. During the evolution of $\mathrm{C}_{4}$ or $\mathrm{C}_{6}$, one-bond coupling of $\mathrm{C}_{4}-\mathrm{C}_{5}$ or $\mathrm{C}_{5}-\mathrm{C}_{6}$ evolves. Thus, the couplings of $\mathrm{C}_{4}-\mathrm{C}_{5}$ or $\mathrm{C}_{5}-\mathrm{C}_{6}$ can be measured readily from the $t_{1}$ dimension $\left(\mathrm{C}_{4}\right.$ or $\left.\mathrm{C}_{6}\right)$ in a well-resolved 3D spectrum. For adenine, the $\mathrm{HCC}(\mathrm{C})$ stands for $\mathrm{H} 2 \mathrm{C} 2 \mathrm{C} 5(\mathrm{C} 4)$ or $\mathrm{H} 2 \mathrm{C} 2 \mathrm{C} 5(\mathrm{C} 6)$, and the couplings of $\mathrm{C}_{4}-\mathrm{C}_{5}$ or $\mathrm{C}_{5}-\mathrm{C}_{6}$ can be measured in a similar fashion as discussed above. In addition, the couplings of $\mathrm{C}_{8}-\mathrm{H}_{8}$ and $\mathrm{C}_{2}-\mathrm{H}_{2}$ can be measured using an IPAP [38] version of $\left[{ }^{1} \mathrm{H},{ }^{13} \mathrm{C}\right]-\mathrm{HSQC}$. The sequences have been demonstrated on both isotropic and anisotropic ${ }^{13} \mathrm{C}$-labeled RNA oligomer ( $\left.24 \mathrm{nt}\right)$ with a concentration of $1.9 \mathrm{mM}$. Details of the measurement precision and shaped pulses can be found in the original work [135].

MQ-HCN-QJ and TROSY-HCN-QJ experiments measure one-bond ${ }^{15} \mathrm{~N}_{1 / 9^{-}}{ }^{13} \mathrm{C}$ RDCs [136]. These quantitative J-correlated MQ HCN experiments focus on the moiety of $\mathrm{H}_{1^{\prime}}-\mathrm{C}_{1^{\prime}}-\mathrm{N}_{1 / 9}$ in nucleic acids. It combines the features of quantitative J correlation [49] with relaxation favored MQ coherence for the measurement of the couplings in an $\mathrm{H}_{1^{\prime}}-\mathrm{C}_{1^{\prime}}$ moiety [132]. The 3D MQ HCN uses an out-and-back scheme, after the magnetization is transferred to $\mathrm{N}_{1 / 9}$. A constant time period (2T) is used for the evolution of ${ }^{15} \mathrm{~N}$ chemical shifts. In the reference experiment, no couplings between ${ }^{15} \mathrm{~N}_{1 / 9}$ and the three attached ${ }^{13} \mathrm{C}$ are evolved. In the J-modulated experiment, only a single coupling between ${ }^{15} \mathrm{~N}_{1 / 9}$ and one of the three attached ${ }^{13} \mathrm{C}$ is active. Thus, the individual one-bond ${ }^{15} \mathrm{~N}_{1 / 9^{-}}{ }^{13} \mathrm{C}$ couplings can be determined from the ratio of peak intensities of the two experiments. Similarly, a TROSY-HCN experiment [132] has been modified to measure the couplings of ${ }^{15} \mathrm{~N}_{1^{-}} \mathrm{C}_{1^{\prime} / 2 / 6}$, and ${ }^{15} \mathrm{~N}_{9}-\mathrm{C}_{1^{\prime} / 4 / 8}$. The sequences have been tested on a $1.5 \mathrm{mM}{ }^{13} \mathrm{C} /{ }^{15} \mathrm{~N}$-labeled 24-nucleotide 
RNA oligomer. There is a detail discussion about the source of measurement errors in the original paper. The one-bond ${ }^{13} \mathrm{C}-{ }^{15} \mathrm{~N}$ couplings can be measured accurately with uncertainties of $\pm 0.5 \mathrm{~Hz}$ provided that the $\mathrm{S} / \mathrm{N}$ ratio is larger than 20:1 in the reference spectra.

\subsection{RDC measurements in ribose and backbone of nucleic acids}

In nucleic acids, the NOE constraints on backbone atoms is sparse. Consequently, the dihedral angles from three-bond $\mathbf{J}$ couplings play important roles in defining the backbone conformation. Recently, quantitative $\mathrm{J}$-modulated $\left[{ }^{1} \mathrm{H},{ }^{13} \mathrm{C}\right]$ CT-HMQC experiments have been proposed to measure ${ }^{3} \mathrm{~J}\left(\mathrm{H} 3^{\prime}, \mathrm{P}\right)[137,138]$. Here, a quantitative $\mathrm{J}$-modulated CT-NOESY ${ }^{31} \mathrm{P}$ difference experiment is used to measure ${ }^{1} \mathrm{H}_{3^{\prime}}{ }^{31} \mathrm{P}$ RDCs [139]. The scheme is composed of a reference and a J-modulated experiment. During the constant time period, the chemical shifts of protons evolve, the couplings between ${ }^{1} \mathrm{H}_{3^{\prime}}$ and other protons are removed by two selective $180^{\circ}$ pulses on ${ }^{1} \mathrm{H}_{3^{\prime}}$, while the couplings between ${ }^{1} \mathrm{H}_{3^{\prime}}$ and ${ }^{31} \mathrm{P}$ are either coupled or decoupled in two separate experiments. Following the $t_{1}$ period, a long NOE mixing time $(300 \mathrm{~ms})$ is used so that the magnetization of ${ }^{1} \mathrm{H}_{3^{\prime}}$ can be transferred to well-dispersed ${ }^{1} \mathrm{H}_{1^{\prime}}$ or base protons. This results in a rather complete set of ${ }^{1} \mathrm{H}_{-}{ }^{31} \mathrm{P}$ couplings. The sequence has been tested on a 12-mer DNA sample. The uncertainties of measured RDCs are less than $0.15 \mathrm{~Hz}$.

3D HCcH-COSY and 3D Relay $\mathrm{HCcH}-\mathrm{COSY}$ measure the couplings of $\mathrm{H}_{2^{\prime}}-\mathrm{C}_{2^{\prime}}$ and $\mathrm{H}_{3^{\prime}}-\mathrm{C}_{3^{\prime}}$ in RNA molecules [140]. The experiments are similar to a constant-time $\mathrm{HCcH}-\mathrm{COSY}$ experiment proposed before [141]. The magnetization transfer scheme is as follows:

$$
{ }^{1} \mathrm{H}_{1^{\prime}}\left(\mathrm{t}_{1}\right) \rightarrow{ }^{13} \mathrm{C}_{1^{\prime}}\left(\mathrm{t}_{2}\right) \rightarrow{ }^{13} \mathrm{C}_{2^{\prime}} \rightarrow{ }^{1} \mathrm{H}_{2^{\prime}}\left(\mathrm{t}_{3}\right)
$$

The experiment makes use of the good dispersion of ${ }^{1} \mathrm{H}_{1^{\prime}}{ }^{1} \mathrm{C}_{1^{\prime}}$ to enhance the resolution of ${ }^{1} \mathrm{H}_{2^{\prime}}$ region. To further reduce the overlap problem, an IPAP filter is used during the last reverse INEPT step so that the components of of ${ }^{1} \mathrm{H}_{2^{\prime}}$ doublet can be separated into two sub-spectra. In the 3D relay $\mathrm{HCcH}-\mathrm{COSY}$ experiment, the magnetization is further relayed to ${ }^{1} \mathrm{C}_{3^{\prime}}$ from ${ }^{1} \mathrm{C}_{2^{\prime}}$ and the signals of ${ }^{1} \mathrm{H}_{3^{\prime}}$ are detected. One advantage of this approach is that no assignment for ${ }^{1} \mathrm{C}_{2^{\prime}}-{ }^{1} \mathrm{H}_{2^{\prime}}$ or ${ }^{1} \mathrm{C}_{3^{\prime \prime}}{ }^{1} \mathrm{H}_{3^{\prime}}$ are required in extracting RDCs for these two vectors as long as the ${ }^{1} \mathrm{C}_{1^{\prime}}{ }^{1} \mathrm{H}_{1^{\prime}}$ can be assigned. The sequence has been tested on a $1.6 \mathrm{mM}{ }^{13} \mathrm{C} /{ }^{15} \mathrm{~N}$ 42-nt RNA sample. The RMS errors are within $2.7 \mathrm{~Hz}$ for RDC values ranging from 32 to $-32 \mathrm{~Hz}$.

$\mathrm{CH}_{2}-\mathrm{S}^{3} \mathrm{E}$ HSQC is a 2D experiment tailored to measure the couplings of germinal ${ }^{1} \mathrm{H}_{A^{-}}{ }^{1} \mathrm{H}_{B}$, and the sum of ${ }^{13} \mathrm{C}-{ }^{1} \mathrm{H}_{A}$ and ${ }^{13} \mathrm{C}_{-}{ }^{1} \mathrm{H}_{B}$ couplings for the $\mathrm{CH}_{2}$ groups in RNAs [142]. There are two novel features in the experiment. Firstly, an $\mathrm{S}^{3} \mathrm{E}$ element is used to separate the upfield and downfield components of a ${ }^{13} \mathrm{C}$ triplet into two sub-spectra. Secondly, when the magnetization is transferred back from ${ }^{13} \mathrm{C}$ to ${ }^{1} \mathrm{H}$, it is selectively transferred from the downfield component of a $\mathrm{CH}_{2}$ triplet into the upfield components of the ${ }^{1} \mathrm{H}_{A^{-}}\left\{{ }^{1} \mathrm{H}_{B}\right\}$ and ${ }^{1} \mathrm{H}_{B}-\left\{{ }^{1} \mathrm{H}_{A}\right\}$ doublets in a way similar to that used in SPITZE-HSQC experiment [119]. Thus, when two spectra are overlaid, E.COSY patterns appear. The displacements along the ${ }^{13} \mathrm{C}$ dimension are the sum of ${ }^{13} \mathrm{C}_{-}{ }^{1} \mathrm{H}_{A}$ and ${ }^{13} \mathrm{C}_{-}{ }^{1} \mathrm{H}_{B}$ couplings, while the displacements along the ${ }^{1} \mathrm{H}$ dimension are the ${ }^{3} \mathrm{~J}_{\mathrm{HA}-\mathrm{HB}}$. The sequence has been applied to a $1.9 \mathrm{mM} 24-\mathrm{nt}{ }^{13} \mathrm{C}$-labeled RNA oligomer to extract the couplings of ${ }^{1} \mathrm{H}_{5^{\prime}}{ }^{1} \mathrm{H}_{5^{\prime \prime}}$ and the sum of ${ }^{13} \mathrm{C}_{5^{\prime}}{ }^{1} \mathrm{H}_{5^{\prime}}$ and ${ }^{13} \mathrm{C}_{5^{\prime}}{ }^{1} \mathrm{H}_{5^{\prime \prime}}$ couplings.

3D H1C1C2 experiment measures five dipolar couplings from an $\mathrm{H}_{1^{\prime}}-\mathrm{C}_{1^{\prime}}-\mathrm{C}_{2^{\prime}}-\mathrm{H}_{2^{\prime}}$ moiety of RNA ribose sugars [143]. It is an E.COSY type experiment with out-and-back style. Starting from $\mathrm{H}_{1^{\prime}}$, the magnetization is transferred to $\mathrm{C}_{2^{\prime}}$ through $\mathrm{C}_{1^{\prime}}$, two constant-time periods are used for chemical shift evolution of $\mathrm{C}_{2^{\prime}}\left(t_{1}\right)$ and $\mathrm{C}_{1^{\prime}}\left(t_{2}\right)$. Protons are not decoupled during both $t_{1}$ and $t_{2}$ periods when the ${ }^{1} \mathrm{H}$ spin-states are also retained. E.COSY patterns appear in the $\left[\mathrm{C}_{1^{\prime}}, \mathrm{C}_{2^{\prime}}\right]$-planes, from which the couplings of $\mathrm{C}_{1^{\prime}}-\mathrm{H}_{1^{\prime}}, \mathrm{C}_{2^{\prime}}-\mathrm{H}_{2^{\prime}}, \mathrm{C}_{1^{\prime}}-\mathrm{H}_{2^{\prime}}$, and $\mathrm{C}_{2^{\prime}}-\mathrm{H}_{1^{\prime}}$ can be extracted. Finally, when magnetization is transferred to $\mathrm{H}_{1^{\prime}}$ from $\mathrm{C}_{1^{\prime}}$, the $\mathrm{H}_{2^{\prime}}$ spin-states are 
not perturbed. $\mathrm{H}_{1^{\prime}}-\mathrm{H}_{2^{\prime}}$ couplings can be measured from the frequency differences in $\mathrm{C}_{2^{\prime}}-\left\{\mathrm{H}_{2^{\prime}}\right\}$ doublets along the $\mathrm{H}_{1^{\prime}}$ dimension. The pulse sequence has been tested on a $1.9 \mathrm{mM}{ }^{13} \mathrm{C}$-labeled RNA 24-nt oligomer. The random errors have been given for five different measured RDCs.

3D $\mathrm{CH}_{2}-\mathrm{S}^{3} \mathrm{CT}$ HBCBCA experiment measures eight couplings for the $\mathrm{C}_{5^{\prime}}-\mathrm{C}_{4^{\prime}}$ group in nucleic acids [127]. Conceptually, the experiment is a modification of the approach used to measure eight couplings for $\mathrm{CH}_{2}$ $\mathrm{CH}$ moiety in proteins. The eight couplings extracted from two $3 \mathrm{D}$ experiments are ${ }^{1} \mathrm{~J}_{\mathrm{C} 4^{\prime}-\mathrm{H} 4^{\prime}},{ }^{2} \mathrm{~J}_{\mathrm{C} 4^{\prime}-\mathrm{H} 5^{\prime+}}$ ${ }^{2} \mathrm{~J}_{\mathrm{C} 4^{\prime}-\mathrm{H} 5^{\prime \prime}},{ }^{2} \mathrm{~J}_{\mathrm{C} 5^{\prime}-\mathrm{H} 4^{\prime}},{ }^{1} \mathrm{~J}_{\mathrm{C} 5^{\prime}-\mathrm{H} 5^{\prime}}+{ }^{1} \mathrm{~J}_{\mathrm{C} 5^{\prime}-\mathrm{H} 5^{\prime \prime}},{ }^{1} \mathrm{~J}_{\mathrm{C} 5^{\prime}-\mathrm{H} 5^{\prime}}-{ }^{2} \mathrm{~J}_{\mathrm{H} 5^{\prime}-\mathrm{H} 5^{\prime \prime}},{ }^{1} \mathrm{~J}_{\mathrm{C} 5^{\prime}-\mathrm{H} 5^{\prime \prime}}-{ }^{2} \mathrm{~J}_{\mathrm{H} 5^{\prime}-\mathrm{H} 5^{\prime \prime},},{ }^{3} \mathrm{~J}_{\mathrm{H} 4^{\prime}-\mathrm{H} 5^{\prime}}$,

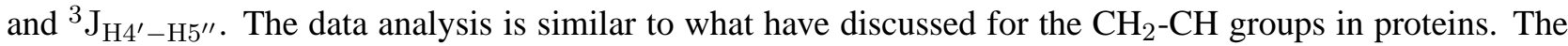
sequence has been demonstrated on a $1.9 \mathrm{mM} 24-\mathrm{nt}{ }^{13} \mathrm{C}$-labeled RNA oligomer, out of 552 possible splitting, 508 can be obtained from an isotropic sample.

\section{Applications of residual dipolar couplings to proteins}

With the advent of tunable alignment media about 8 years ago, RDCs have been applied to study an increasing number of problems in biochemistry and structural biology ranging from automated backbone resonance assignment, structure determination, protein folding to ligand-protein and protein-protein interactions. In the following we will focus on the application of RDCs to study proteins. However, we will not discuss the application of RDCs to study protein dynamics in solution since that topic was reviewed extensively just a few years ago [11].

\subsection{Automated backbone resonance assignment}

Chemical shift resonance assignment is a prerequisite for applying NMR spectroscopy to study proteins and nucleic acids in solution such as structure determination, the characterization of dynamics and ligand-protein, protein-protein interactions, and the elaboration of the mechanism of enzyme catalysis. The automation of resonance assignment is highly desirable since manual assignments can be very time-consuming. The traditional approaches for automated assignment focus on either the through-bond triple-resonance or throughspace NOE experiments. Recently, several algorithms have been developed to assign the resonances of backbone nuclei using either RDCs alone or RDCs in combination with the NMR data used for the traditional approaches. These RDC-based automated assignment algorithms all require that either there exists an a priori structure $[144,145,146]$ or intermediate structures can be determined on-the-fly $[147,148,149,150]$.

\subsubsection{Assignment requiring an a priori structure}

A bipartite-matching ${ }^{3}$-based protocol developed by Hus and coworkers [145] for backbone resonance assignment uses both NH RDCs and $\mathrm{C}_{\alpha}, \mathrm{C}_{\beta}$ chemical shifts but does not rely on any sequential NMR connectivity information. The algorithm requires ${ }^{15} \mathrm{~N}$ and ${ }^{13} \mathrm{C}$ doubly-labeled samples and triple-resonance experiments for $\mathrm{C}_{\alpha}$ and $\mathrm{C}_{\beta}$ chemical shifts. The assignment problem is formulated in terms of a weighted bipartite-matching problem that can be solved in $O\left(n^{3}\right)$ time, where $n$ is the number of residues. The weight for bipartite-match is set to be

$$
\chi^{2}=\sum_{1}^{n} C(i, \pi(i)), \quad C(i, j)=C_{r}(i, j)+K C_{s}(i, j)
$$

where $\pi(i)$ is the permutation of $1,2, \ldots, n, C_{r}(i, j)$ and $C_{s}(i, j)$ are, respectively, the differences between the experimental RDCs and the RDCs back-computed from the a prior structure using Eq. (11), and the experi-

\footnotetext{
${ }^{3}$ A bipartite graph is a graph $G=(V, E)$ with a set of nodes $L$ and a set of nodes $R$ such that $L \cap R=\emptyset, L \cup R=V$, and for all edges $(u, v) \in E, u \in L$ and $v \in R$. A bipartite-matching is a subset of edges of $M \subseteq E$ such that for all vertices $v \in V$, at most one edge of $M$ is incident on $v$. A maximum bipartite-matching is a matching of maximum cardinality [151].
} 
mental chemical shifts and the average values for the corresponding amino acids in the BMRB database. The $K$ is the relative weight. The matrix $\mathbf{C}$ or $\mathbf{C}_{r}$ or $\mathbf{C}_{s}$ in Eq. (14) with elements $C(i, j)$ or $C_{r}(i, j)$ or $C_{s}(i, j)$ is called a cost matrix. The key steps of the algorithm are shown in Fig. (11). Though the bipartite-matching can

1. Convert experimental $\mathrm{C}_{\alpha}$ and $\mathrm{C}_{\beta}$ chemical shifts into cost matrix $\mathbf{C}_{s}$

2. Estimate from RDCs the initial values for axial and rhombic components of the alignment tensor, $D_{a}$ and $R$.

3. WHILE convergence has not been reached,

(a) Choose new Euler angles $\alpha, \beta, \gamma$.

(b) Compute the cost matrix $\mathbf{C}$ based on the a prior structure and chemical-shift difference.

(c) Solve the weighted matching problem.

(d) Define the subset of peptide planes that show good agreement between experimental RDCs and the RDCs predicted from the a prior structure.

(e) Refine Euler angles $\alpha, \beta, \gamma$ and the alignment tensor $D_{a}$ and $R$ values on this subset with the assignment obtained in step c.

(f) Compute a new cost matrix $\mathbf{C}$.

(g) Solve weighted matching problem.

4. END WHILE

Figure 11: A bipartite-matching algorithm for backbone resonance assignment. Grid-search is used for choosing Euler angles $\alpha, \beta, \gamma$ in steps 3(a) and 3(e).

be solved in $O\left(n^{3}\right)$ time, the complexity of the entire algorithm is actually more complicated since bipartitematching is performed for each grid-point for three Euler angles $\alpha, \beta, \gamma$, and it is not guaranteed that $D_{a}$ and $R$ values can be estimated accurately. Thus, the minimum weight computed by the algorithm is not guaranteed to be the real global minimum. In addition, since the assignment problem is formulated as a bipartite-matching problem, adding any sequential-connectivity information will make the problem NP-hard ${ }^{4}$, which means that it is difficult to extend the protocol to incorporate such information. The protocol has been applied to human ubiquitin using two or three RDCs per residue measured in a single medium (Pfl phage medium) together with $\mathrm{C}_{\alpha}$ and $\mathrm{C}_{\beta}$ chemical shifts. The algorithm yields the correct assignment for more than $90 \%$ of the protein backbone resonances.

The NP-hardness of bipartite-matching with (sequential connectivity) restraints was solved approximately in the program Nuclear Vector Replacement (NVR) [144] using expectation/maximization (E/M) method 5. NVR is built upon chemical-shift prediction algorithms (SHIFTS and SHIFTX) and bipartite-matching algorithm to perform assignment using RDCs measured in two aligning media in combination with unambiguous backbone $\mathrm{H}_{\mathrm{N}}-\mathrm{H}_{\mathrm{N}}$ NOE restraints $\left(d_{N N}\right)$ and amide-proton exchange (HD) data. HD data is used to identify surface residues. NVR is divided into two phases: tensor determination and resonance assignment phases. In the first phase, chemical shift predictions from the BMRB database, SHIFTS and SHIFTX, $d_{N N}$ restraints, and HD data are used to make a small number of assignments using an E/M method. Specifically, this phase attempts to assign at least 5 peaks for the purpose of determining the two alignment tensors directly by singular value decomposition (SVD) [153]. The estimated tensors are then used to convert RDCs into probabilistic constraints in the resonance assignment phase. Seven bipartite-graphs were built in the second phase: $B_{H D}, B_{B M R B}, B_{N O E}, B_{S H I F T S}, B_{S H I F T X}, B_{R D C 1}$ and $B_{R D C 2}$. The six graphs

\footnotetext{
${ }^{4} \mathrm{NP}$-hard means that the running time of the algorithm increases exponentially with the size of the input.

${ }^{5} \mathrm{E} / \mathrm{M}$ is an algorithm for computing maximum likelihood estimates from incomplete data [152]. The maximum returned from an $\mathrm{E} / \mathrm{M}$ algorithm is not guaranteed to be the global maximum.
} 
$B_{H D}, B_{B M R B}, B_{S H I F T S}, B_{S H I F T X}, B_{R D C 1}$ and $B_{R D C 2}$ were then synchronized and used in an E/M step for updating the assignment by the maximum-bipartite-matching. However, $B_{N O E}$ was used after the E/M step to make more assignment using $d_{N N}$ restraints since the maximum-bipartite-matching algorithm can not handle $d_{N N}$ restraints. This step is called Apply-NOE step. Each cycle of the E/M step followed by the Apply-NOE step made at least one assignment. The algorithm stops when all the residues have been assigned. The alignment tensors were also updated in each cycle of the E/M step followed by the Apply-NOE step. The a priori structure required by NVR can be either an x-ray structure with the identical protein sequence or homologous structure. The algorithm requires only uniform ${ }^{15} \mathrm{~N}$-labeled sample and ${ }^{1} \mathrm{H}_{-}{ }^{15} \mathrm{~N}$ HSQC spectrum, ${ }^{1} \mathrm{H}_{-}{ }^{15} \mathrm{~N}$ RDCs, and sparse backbone $d_{\mathrm{NN}}$ restraints from a 3D ${ }^{15} \mathrm{~N}$-edited NOESY spectrum. NVR runs in minutes and can efficiently assign the ${ }^{1} \mathrm{H}_{-}{ }^{15} \mathrm{~N}$ backbone resonances as well as the sparse NOE restraints $d_{\mathrm{NN}}$ from the 3D ${ }^{15} \mathrm{~N}-\mathrm{NOESY}$ spectrum, in $O\left(n^{3}\right)$ time. Though the best solution obtained from NVR is not guaranteed to be the real global minimum since E/M can only achieve local maximum, NVR performed rather well in practice. NVR has been tested on several proteins: human ubiquitin (76 residues), hen lysozyme (129 residues) and streptococcal protein G (56 residues) where for ubiquitin, RDCs were matched to four different structures, including one mutant (homolog). NVR achieved an average assignment accuracy of over 99 $\%$ when the chemical shift errors for amide proton ${ }^{1} \mathrm{H}_{\mathrm{N}}$ and amide nitrogen ${ }^{15} \mathrm{~N}$ were set to be, respectively, $\pm 0.01 \mathrm{ppm}$ and $\pm 0.1 \mathrm{ppm}$.

Jung and coworkers have developed an ad-hoc algorithm, MARS [154, 146], for sequential assignment of backbone resonances based on (a) the chemical shift predication obtained via a secondary structure prediction method, (b) the identification of amino acid types from the chemical shifts for $\mathrm{C}_{\alpha}, \mathrm{C}_{\beta}$ and $\mathrm{C}^{\prime}$. MARS requires an ${ }^{15} \mathrm{~N}$ and ${ }^{13} \mathrm{C}$ doubly-labeled sample and triple-resonance experiments. The original MARS algorithm [154] was extended to incorporate RDCs where RDCs are used essentially for filtering out the false positives in connectivity obtained from the original MARS using chemical shift information alone. In case of small proteins and with three RDCs per residue, MARS [146] can assign more than $90 \%$ of backbone resonances with an assignment reliability of $56 \%$ without the need for sequential connectivity information. For bigger proteins, the combination of sequential connectivity information with RDC-matching enables more residues to be assigned. In MARS, RDC-enhanced assignment is particularly useful when chemical shifts and sequential connectivity only provide a few reliable assignments. Since there are many manual steps and the performance of the algorithm depends critically on a set of parameters such as the error ranges set for $\mathrm{C}_{\alpha}, \mathrm{C}_{\beta}$ chemical shifts, the worst-time complexity ${ }^{6}$ of MARS is most likely to be exponential. For examples, in practice, the running time for malate synthase $\mathrm{G}$ (723 residues) vary from 2 hours using $\mathrm{C}_{\alpha}, \mathrm{C}_{\beta}$, C' connectivity with the thresholds of $0.2,0.4$ and $0.15 \mathrm{ppm}$ for $\mathrm{C}_{\alpha}, \mathrm{C}_{\beta}, \mathrm{C}^{\prime}$, respectively, to 13 hours using only $\mathrm{C}_{\alpha}, \mathrm{C}_{\alpha}$ connectivity with thresholds of 0.2 and $0.4 \mathrm{ppm}$, respectively, and up to 150 hours when only $\mathrm{C}_{\alpha}, \mathrm{C}_{\beta}$ connectivity information is available with a resolution of 0.5 and $0.5 \mathrm{ppm}$, respectively. Since RDCs is used only for enhancing the reliability, the extension of MARS to RDCs is also expected to be exponential in the worst-case. MARS with RDCs requires an a prior structure for matching the experimental RDCs with the RDCs back-computed from the $a$ prior structure.

\subsubsection{Simultaneous assignment and structure determination}

Tian and coworkers [148] have developed a protocol for simultaneous backbone resonance assignment and structure determination with the intermediate structures computed on-the-fly. The protocol requires chemical shifts of protons, nitrogen and carbons from triple-resonance experiments. Six RDCs per residue in two media (12 RDCs per residue) were used to compute the structures and filter the false connections obtained from the chemical shifts of $\mathbf{C}_{\alpha}$ and $\mathbf{C}_{\beta}$ alone. The $(\phi, \psi)$ angles connecting a pair of peptide planes were systematically varied and the best $(\phi, \psi)$ values were selected by comparing the back-computed RDCs with the 12 RDCs

\footnotetext{
${ }^{6}$ The worst-time complexity means the longest possible running time of the algorithm with particular inputs.
} 
(six in each medium) in each peptide plane (nine unique for a pair of planes). Additional peptides were then added along with other RDCs (3-6 RDCs) to extend the fragments to lengths of 6-16 residues. In the case of a small protein rubredoxin (54 residues), geometries for six fragments, each separated by a single proline, were identified. These fragments were then combined using the fact that fragments must be oriented so that the principal order frames as viewed from each fragment must coincide. In most cases, bonding restrictions imposed by the single connecting proline provided unique translational positioning. RDC data from a second medium helped resolve any remaining 4-fold orientational degeneracy of the individual order frames. This brute-force algorithm is exponential with the number of residues since it varies $(\phi, \psi)$ angles of each residue systematically by grid-search. The algorithm was tested on a $4.5 \mathrm{mM}{ }^{15} \mathrm{~N}$-labeled rubredoxin while ${ }^{13} \mathrm{C}$ experiments were performed on natural abundance. For the collection of the six types of RDC data, a phase-modulated HSQC experiment [51] (Section 3.1), a soft HNCA-E.COSY experiment [117] (Section 3.7) and a 2D IPHSQC experiment [112] (Section 3.7) were selected. Both the original HNCA-E.COSY pulse sequence and the IP-HSQC pulse sequence were modified for acquiring dipolar couplings from natural abundance ${ }^{13} \mathrm{C}$ protein samples.

Jens and Baker have also developed an automated simultaneous resonance assignment and structure determination approach $[149,150]$ using RDCs in combination with NOEs and chemical shifts. The original version [149] relies on a de novo protein structure prediction algorithm, ROSETTA and a Monte-Carlo procedure that searches for the assignment of resonances to atoms that produces the best fit to the experimental NMR data to candidate 3D structures from ROSETTA. A large ensemble of models is generated from sequence information alone by ROSETTA, an optimal assignment is identified for each model, and the models are then ranked based on their fit with the NMR data using the previously identified assignments. The original method was tested on nine protein sequences between 56 and 140 amino acids and published chemical shift (CS), NOE, and RDC data. The procedure yielded models with backbone RMSD between $3 \AA$ and $6 \AA$, and, in four of the nine cases, the partial assignments obtained by the method could be used to refine the structures to high resolution (0.6-1.8 $\AA$ ) by repeated cycles of structure generation guided by the partial assignments, followed by reassignment using the newly generated models. With the published NOEs, the distances given in the NOE lists were replaced with intensities computed from $\mathrm{Cr}^{-6}$ where $C$ and $r$ are, respectively, a constant and the internuclear distance. However, it is not clear from the paper how the NOE intensity is generated if only a range of distances, rather than a single distance is reported. When the protocol is applied to real NMR data acquired on a 140 amino acids fumarate sensor, DcuS, the original approach can only generate a fold with a $6.0 \AA$ backbone RMSD with respect to the structure determined by either a traditional NMR method or x-ray diffraction. The real NMR data consists of 1,100 chemical shift (CS) signals, 3,000 NOESY cross peak intensities, and 209 RDCs extracted directly from the original spectra. After both the x-ray and NMR structures become available, the approach was modified by weighting the assignment against a wide range of models generated from various structure prediction techniques [150], in addition to those models generated from ROSETTA. The new procedure improves both the accuracy and assignment. With the new protocol, the backbone fold was refined to an accuracy of $3.7 \AA$ RMSD from a previous $6.0 \AA$ RMSD obtained from the original protocol.

An approach called CAP has also been developed for simultaneous assignment and tertiary structure determination of RNAs [147]. The approach has been demonstrated on a uniformly ${ }^{15} \mathrm{~N}-{ }^{13} \mathrm{C}$ doubly-labeled 27 nt variant of the trans-activation response element (TAR) RNA from HIV-I. The approach involves examination of all the allowed sequence-specific resonance assignment permutations for best-fit agreement between measured RDCs and RDCs back-computed from the coordinates for sub-structures in a target RNA. Using idealized A-form geometries to model Watson-Crick helices and coordinates from a previous X-ray structure to model a hairpin loop in TAR, the best-fit RDC assignment solutions are determined very rapidly $(<$ five minutes of computational time) and are in agreement with the corresponding NOE-based assignments. Orien- 
tational constraints derived from RDCs are used to assemble sub-structures into an RNA tertiary conformation. The approach can assign the resonance in minutes for the $27 \mathrm{nt}$ RNA though the algorithm is exponential in the worst-case. More specifically, the exhaustive search performed by CAP over all permutations makes it difficult to scale up to larger RNAs.

\subsection{Structure determination using RDCs}

Three dimensional structures are critical for understanding biochemical functions of proteins at atomic level, structure-based drug design, and genomic annotation. The application of RDCs to structure determination can be classified into three categories based on the roles RDCs played in the computation:

1. Refinement by RDCs of structures computed mainly from NOE and other NMR restraints.

2. De novo structure determination using RDCs in combination with other NMR restraints.

3. De novo structure determination using RDCs alone.

These algorithms also differ in how all the conformations consistent with the experimental data are searched. The main search methods are the following:

1. Simulated annealing (SA) in combination with molecular-dynamics (MD) simulation.

2. Monte-Carlo method for assembling the structural fragments ${ }^{7}$ obtained from the PDB by best matching the experimental RDCs with all the fragments in the PDB.

3. Systematical search over all the possible conformations determined by backbone dihedral angles computed directly from RDCs.

For the structure determination of native proteins, the goal of the search is to find a conformation which has the minimum pseudoenergy. The terms for the pseudoenergy also differ greatly in different algorithms with $\mathrm{MD} / \mathrm{SA}$ has the most elaborate energy terms including all the molecular mechanics energy terms and pseudoenergy terms for various NMR restraints. The pseudoenergy terms in the last two approaches are much simpler. The results of the applications of various algorithms to experimental NMR data were summarized in Table 2. Those algorithms for simultaneous resonance assignment and structure determination have been described above and will not be repeated here.

\subsubsection{Refinement}

RDCs were first applied to refine NMR structures computed using mainly NOE distance restraints and scalar couplings by restrained molecular dynamics (MD) in combination with simulated annealing (SA) approach for searching the conformational space. In these approaches, RDCs are incorporated as an additional pseudoenergy term $[155,156]$ while the relative orientation between the alignment tensor and the molecular frame is kept in flow during the structure computation. The Saupe elements (alignment tensor) are either estimated by grid-search [93] or from RDC powder pattern [94] obtained from multiple RDCs measured in a single aligning medium. The quality of structures was improved greatly by the addition of several RDC restraints per residue [156]. Up to now, refinement remains to be the most frequent application of RDCs to protein structure determination.

\subsubsection{RDC in combination with other NMR restraints}

Several research groups $[157,158,159]$ have developed different approaches to compute structures using mainly backbone RDCs in combination with a relatively small amount of NOE distance restraints and scalar couplings. In the input, the amount of NMR restraints other than RDCs were, typically, $<7$ per residue. Brown

\footnotetext{
${ }^{7}$ A structural fragment consists of $m$ consecutive residues ( $m \geq 7$ in general).
} 
and coworkers [157] has applied MD/SA to compute the fold of human ubiquitin using more than three RDCs per residue in two media in combination with backbone $\mathrm{H}_{\mathrm{N}}-\mathrm{H}_{\mathrm{N}}$ NOEs. Using the MD/SA approach implemented in XPLOR/CNS to search the conformational space, the computed structures have $1.40 \AA$ backbone RMSD with respect to the X-ray structure [73] (Table 2). Using both RDCs and sparse NOEs (about 7 NOEs per residue), Mueller and coworkers [158] computed the global fold of maltose-binding protein (MBP, 370 residues) in complex with the substrate beta-cyclodextrin. Initial structures were calculated using XPLOR/CNS with default parameters on the basis of $1943 \mathrm{NOE}$ distance restraints of $\mathrm{H}_{\mathrm{N}}-\mathrm{H}_{\mathrm{N}}, \mathrm{H}_{\mathrm{N}}-\mathrm{CH}_{3}$ and $\mathrm{CH}_{3}-\mathrm{CH}_{3}, 48$ hydrogen bond and 555 dihedral angle restraints. The computed structures have large numbers of violations of RDC restraints, with distortion of peptide plane geometry. Next, the structure was refined using one-bond $\mathrm{HN}, \mathrm{C}_{\alpha}-\mathrm{C}^{\prime}, \mathrm{N}-\mathrm{C}^{\prime}$, two-bond $\mathrm{H}_{\mathrm{N}}-\mathrm{C}^{\prime}$ and three-bond $\mathrm{H}_{\mathrm{N}}-\mathrm{C}_{\alpha}$ RDCs to compute the discrete orientations of each peptide plane. These RDCs were measured using the methyl-protonated, ${ }^{15} \mathrm{~N},{ }^{13} \mathrm{C},{ }^{2} \mathrm{H}$-labeled MBP sample with TROSY-based HNCO pulse sequences as described in [77] (Section 3.1). The orientation which best matches that in the initial NMR structures calculated from NOE and dihedral angle restraints exclusively is used to refine further the structures using a new module written for the program XPLOR/CNS. When applied to experimental data recorded on MBP, the precision of the family of structures generated improves from 5.5 $\AA$ to $2.2 \AA$, while the backbone RMSD with respect to the X-ray structure is reduced from $5.1 \AA$ to $3.3 \AA$. The protocol was further improved [159] by direct refinement of structures against measured dipolar couplings and the changes in anisotropic carbonyl chemical shifts upon alignment using MD/SA in torsion-angle space (torsion angle dynamics protocol in XPLOR/CNS).

\subsubsection{RDC alone}

Recently, approaches have been developed to determine backbone structures or folds using RDC data alone or in combination with very sparse NOEs. The approaches include RDC-restrained MD [157, 160], molecular fragment replacement (MFR) methods [161, 24, 162, 163], and positioning of ideal $\alpha$-helices oriented by RDCs [164].

Hus and coworkers [160] employed a least-square fitting procedure followed by MD/SA to compute a backbone structure using five RDCs per residue in two media. All the backbone $(\phi, \psi)$ angles, together with the seven independent Saupe elements required to convert the RDC data into orientational restraints, were estimated by a least-square fitting procedure with many variables (more than 100 variables even for the small protein human ubiquitin). It is not guaranteed that the least-square fitting procedure can find the real global minimum and thus the Saupe matrices and $(\phi, \psi)$ angles may have large errors. However, the next MD/SA step may greatly reduce these errors. Baker and coworkers [162] have developed a database-based approach ROSETTA-NMR where they incorporated RDCs into their ab initio structure prediction method ROSETTA. In RosetTA-NMR, RDCs were first employed to select structural fragments from the PDB. The whole structures constructed from the chosen fragments were further refined by ROSETTA using a Monte-Carlo method. In principle, their method can work with any number of RDCs per residue but the accuracy with fewer than two RDCs per residue was relatively poor (Table 2). All these algorithms use heuristic methods such as simulated annealing [157, 160] or Monte-Carlo simulation [162] to find a solution. The fragment replacement method was pioneered by Bax and coworkers [24]; their method used five RDCs per residue in two media plus chemical shifts to select seven-residue fragments from the PDB. Following the same idea, Andrec and coworkers [161] have developed an approach to compute the fold of ubiquitin using five RDCs per residue measured in a single medium. The key feature of their approach is that they employed a filter that requires overlapping fragments to select the same structural form. The approach was tested with ubiquitin. Compared with the fold for ubiquitin computed from five RDCs per residue in two media, the fold by their approach was less accurate. A different approach was demonstrated by Prestegard and coworkers [164], where RDCs were used only to orient ideal $\alpha$-helices. However, in their study the helices were not refined any further using the experimental RDCs, and no $\beta$-sheet structure was computed. 


\begin{tabular}{|l|c|c|c|c|c|c|}
\hline Protein & $\alpha / \beta$ residues & RDCs & Type of RDCs & Hydrogen bonds & NOEs & RMSD \\
\hline Ubiquitin & $39 / 75$ & 78 & $\mathrm{NH}, \mathrm{NH}$ in two media & 12 & 4 & $1.23 \AA$ \\
\hline Ubiquitin & $41 / 75$ & 76 & $\mathrm{NH}, \mathrm{CH}$ in one medium & 12 & 4 & $0.97 \AA$ \\
\hline Dini & $41 / 81$ & 75 & $\mathrm{NH}, \mathrm{CH}$ in two media & 6 & 9 & $1.55 \AA$ \\
\hline Dini & $41 / 81$ & 80 & $\mathrm{NH}, \mathrm{C}_{\alpha} \mathrm{C}^{\prime}$ in one medium & 6 & 9 & $1.35 \AA$ \\
\hline Protein $\mathrm{G}$ & $29 / 56$ & 53 & $\mathrm{NH}, \mathrm{NH}$ in two media & 9 & 4 & $0.98 \AA$ \\
\hline Protein $\mathrm{G}$ & $33 / 56$ & 61 & $\mathrm{NH}, \mathrm{C}_{\alpha} \mathrm{C}^{\prime}$ in one medium & 9 & 4 & $1.30 \AA$ \\
\hline
\end{tabular}

Table 1: Results of our algorithm on 3 proteins; experimental RDC data for Ubiquitin, Dini and Protein $\mathrm{G}$ was taken from the BioMagResBank (BMRB). The second column indicates how many residues were in either $\alpha$-helicesor $\beta$-sheets. The given RMSD values were computed by comparing the oriented and translated secondary structure elements computed by our algorithm to existing structures. Ubiquitin to a high-resolution X-ray structure; Dini to one NMR structure; and Protein G to on NMR structure (with no $\beta$-strands).

\begin{tabular}{|l|c|c|c|c|}
\hline Programs & Techniques & Number of Restraints Per Residue & Accuracy & References \\
\hline Xplor/CNS & MD/SA & 6 RDCs & $1.40 \AA$ & {$[157]$.} \\
\hline SCULPTOR & MD/SA & 11 RDCs, & $1.00 \AA$ & {$[160]$} \\
\hline MFR & Database & 10 RDCs, 5 Chemical shifts & $1.21 \AA$ & {$[24]$} \\
\hline Rosetta-NMR & DataBase/MC & 3 RDCS, 5 Chemical shifts & $1.65 \AA$ & {$[162]$} \\
\hline Rosetta-NMR & DataBase/MC & 1 RDCS & $2.75 \AA$ & {$[162]$} \\
\hline & Exact solution/Systematic search & 2 RDCs & $1.45 \AA$ & {$[25]$} \\
\hline
\end{tabular}

Table 2: Comparison of the ubiquitin backbone structure computed by our algorithm with the best structures computed by existing heuristic approaches.

Lincong and coworkers have developed ab-initio algorithms [25, 82, 165, 166] for computing backbone structures using a few NOEs and 2 RDCs per residue in one or two different aligning media. Specifically, the algorithms (Fig. 12) compute the conformation and global orientations of individual secondary structure elements, independently, by an exact solution, systematic search-based minimization method using only 2 RDCs per residue. The systematic search (Fig. 13) is built upon quartic equations for computing, exactly and in constant time, the directions of an internuclear vector from RDCs, and linear or quadratic equations for computing the sines and cosines of backbone dihedral $(\phi, \psi)$ angles from two vectors in consecutive peptide planes. In contrast to heuristic search such as SA or MC used by other NMR structure determination algorithms, the minimization algorithm can be analyzed rigorously in terms of expected algorithmic complexity and the coordinate precision of the protein structure as a function of error in the input (experimental) data. The algorithm has been successfully applied to compute the backbone structures of three proteins (Table 1) using experimental NMR data. Compared with MD/SA-based or database-based methods, the results demonstrated that the exact solution and systematic search based algorithms can achieve similar or better accuracy (Table 2).

\subsubsection{Fold recognition by RDCs}

Yu and coworkers [167] have developed a computer program, RDC-PROSPECT, for the identification of a structural homolog or analog of a target protein in the PDB, which best matches the ${ }^{1} \mathrm{H}-{ }^{15} \mathrm{~N}$ RDC data of the protein recorded in a single ordering medium. The identified structural homolog/analog can then be used as a starting model for RDC-based structure calculation. Since RDC-PROSPECT uses only RDC data and secondary structure information predicted from protein sequence by the program PSIPRED, its performance is virtually independent of sequence similarity between a target protein and its structural homolog/analog, making it applicable to protein targets out of the scope of current protein threading techniques. RDC-PROSPECT 


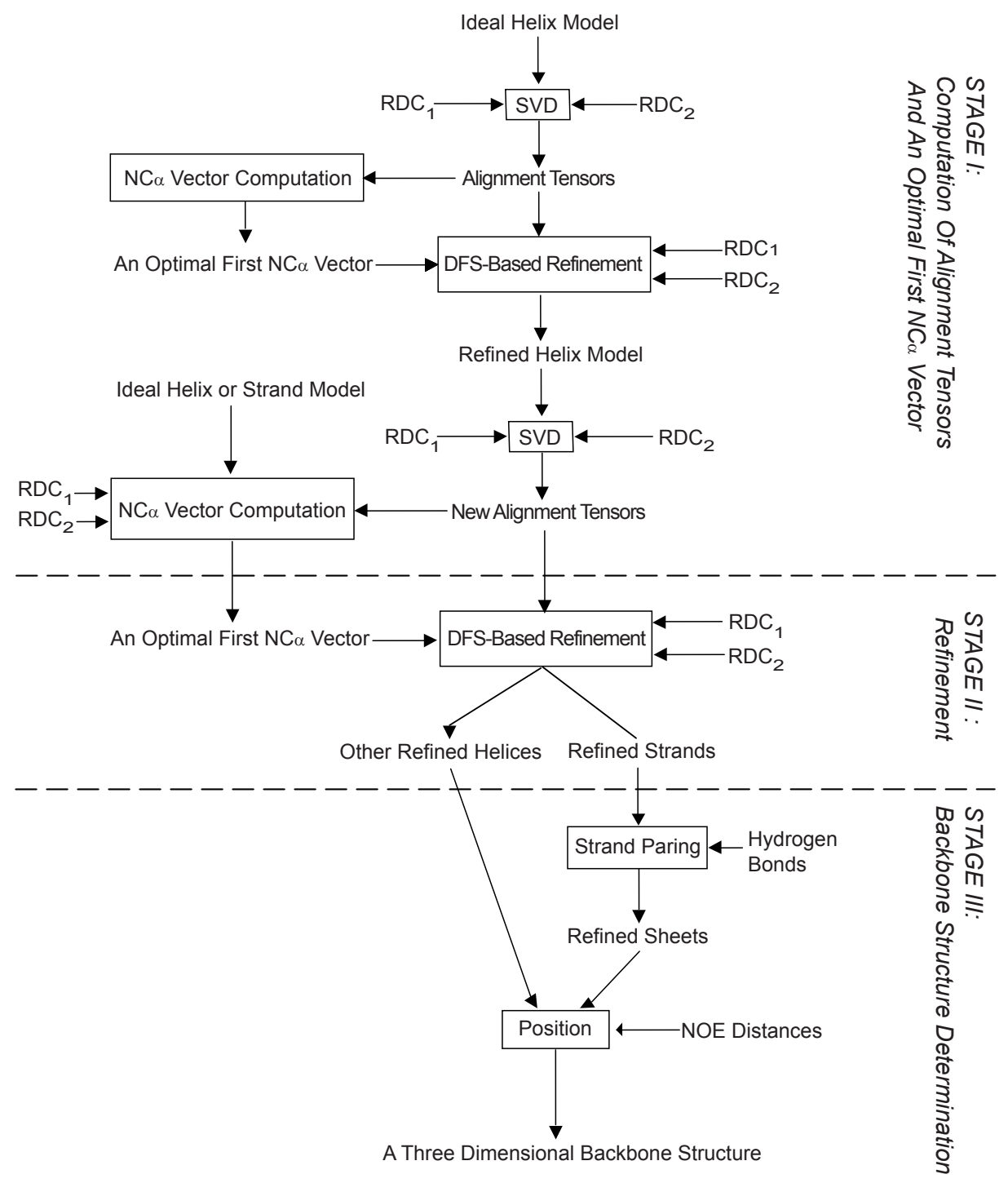

Figure 12: A flow chart illustrating the algorithm. A chart illustrating the three major stages (delimited by two dashed lines) of the algorithm beginning with the input RDC data and ideal helix and strand models to a final three dimensional backbone structure. RDCs refer to RDCs measured in two media. As stated in the main text, stage I is an iterative process for refining the helix and computing alignment tensors. DFS refers to depth-first search and SVD refers to singular value decomposition. Reprinted from [25], with permission from Springer Science and Business Media. 


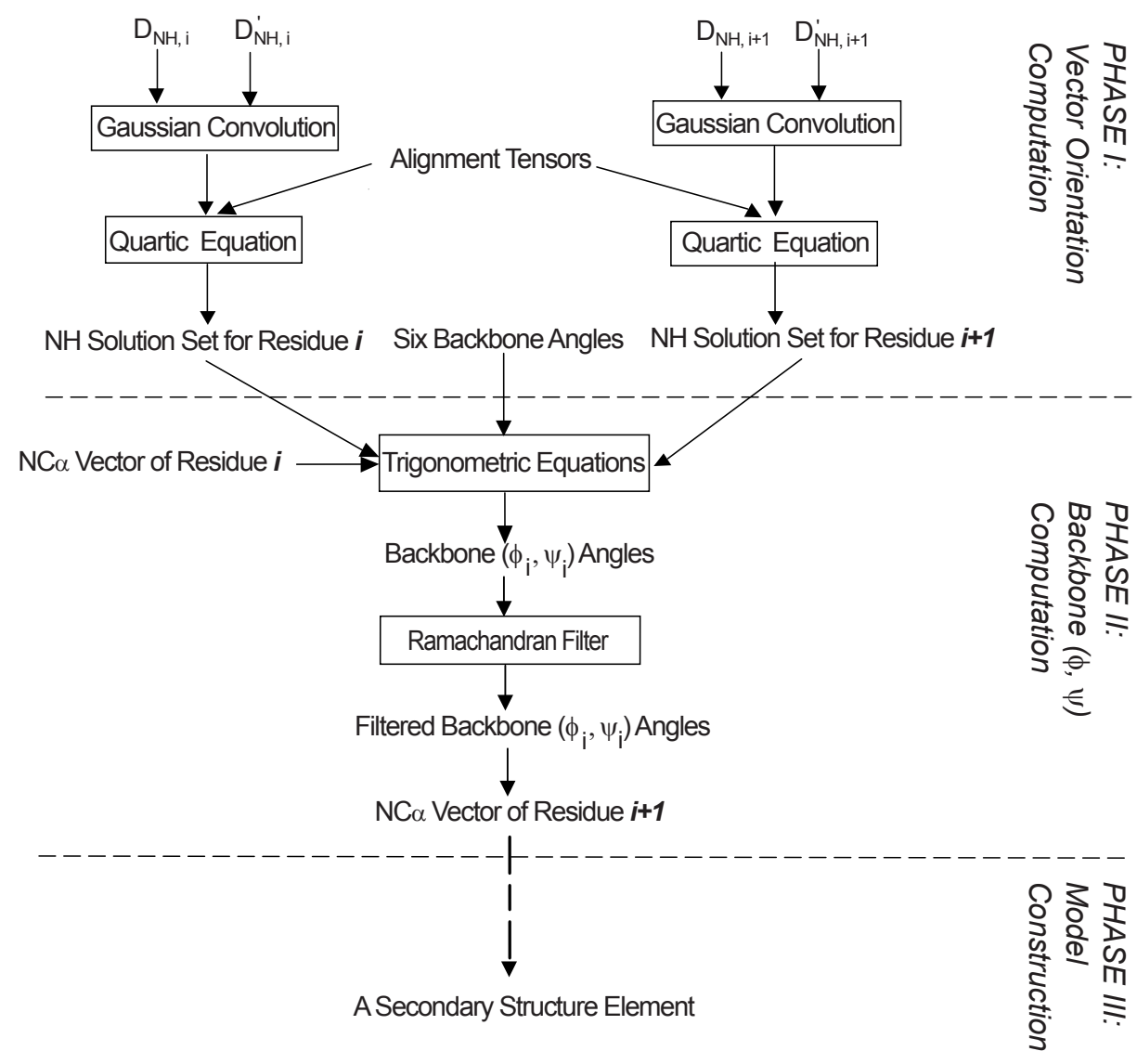

Figure 13: A flow chart of a single step of the DFS-based refinement stage. The three major phases are delimited by two dashed lines. Please refer to the original paper [25] for the definitions of the six angles, the quartic and trigonometric equations. The Ramachandran filter represents filtering through the favorable Ramachandran regions and the Gaussian convolution corresponds to the simulation of the experimental error by a Gaussian distribution with the experimental RDC values in two different media $\left(D_{N H, i}\right.$ or $\left.D_{N H, i}^{\prime}\right)$ as its mean and the error as its variance. Many such steps are required for refining a secondary structure element as shown by a dashed arrow. Reprinted from [25], with permission from Springer Science and Business Media. 
identifies a structural fold through finding a template fold in the PDB, which best aligns with NH RDC data, secondary structure prediction and gap penalty. The best alignment here means to minimize the following scoring function:

$$
\sum\left[\omega_{1} \frac{\left|r_{i}-r_{A(i)}^{\prime}\right|}{\sigma}+\omega_{2} M\left(S_{i}, S_{A(i)}^{\prime}\right)\right]+\sum p G_{j}
$$

where $r_{i}$ and $r_{A(i)}^{\prime}$ are, respectively, the experimental RDC and the RDC back-computed from the aligned template structure $A(i)$ for residue $i ; S_{i}$ and $S_{A(i)}^{\prime}$ are, respectively, the predicted secondary structure type of position $i$ of the target protein and the assigned secondary structure of position $A(i)$ of the template structure and $M\left(S_{i}, S_{A(i)}^{\prime}\right)$ is a function of secondary structures; $p G_{j}$ is the gap penalty for the $j$ th gap in the alignment; $\omega_{1}$ and $\omega_{2}$ are weights. The minimization is achieved by a dynamic programming (DP) ${ }^{8}$. The RDC-PROSPECT program has been tested on all ${ }^{1} \mathrm{H}_{-}{ }^{15} \mathrm{~N}$ RDC data (representing 33 proteins) available in the BMRB database and the literature (by October, 2003). The program correctly identified the structural folds for approximately $80 \%$ of the target proteins, significantly better than previously reported results, and achieved an average alignment accuracy of $97.9 \%$ residues within 4-residue shift. The authors claimed that RDC-PROSPECT is at least one order of magnitude faster than previously reported algorithms for principal order frame search, making the algorithm fast enough for large-scale applications.

Meiler and coworkers [168] have developed a program, DipoCoup, for structural homology search using RDCs in combination with secondary structure prediction based on NMR chemical shifts. Annila and coworkers [169] are the first to use assigned RDC to search for structural homologs, while Andrec and coworkers have also developed an approach to recognize the structural motif using RDCs [170]. More recently, Langmead and Bruce have developed an approach for identifying protein folds from sequences using unassigned RDCs [171]. The interested readers can refer to their original papers for the details.

\subsection{Protein folding}

A very interesting recent application of RDCs is to study the structures of denatured proteins and the protein folding process. Understanding how a protein can fold, in less than a second, from the denatured state to the native state, is a fundamental problem in structural biology. Such knowledge also has biochemical applications ranging from structure prediction to protein design. Moreover, it may provide clues to the causes of certain diseases such as Alzheimer's, which appears to involve misfolding of the amyloid protein. Though much progress has been made in understanding the folding process in recent years, some fundamental questions remain. One of them is the distribution of structures in the denatured state $[172,173,174]$. In contrast to the native state where a single conformation dominates, the denatured state appears to be an ensemble of heterogeneous random coils $[175,172]$, a conclusion reached through the measurement of radius of gyration by small-angle X-ray scattering [176]. The quantification of such structure distribution will be critical for answering two key questions in protein-folding: (a) are the denatured structures random-coils? and (b) are the denatured structures native-like? If stable nativelike topologies persist on unfold states as claimed by some studies using RDC data measured in the denatured state [177, 178, 179], it may be straightforward to explain the rapid refolding kinetics displayed by many proteins. However, if no such native-like topology exists in the denatured state [180,181, 182], some unknown mechanisms must be responsible for the observed fast rates of refolding. RDCs measured in the denatured state may provide quantitative answers to these perplexing questions.

\footnotetext{
${ }^{8}$ Dynamic programming [151] is a computational technique for finding a global extreme (minimum or maximum) for those optimization problems where the problem can be solved, recursively, through the solutions to the subproblems of the same form and the original problem can be solved by using the solutions to the subproblems many times. In dynamic programming, the solutions to the subproblems are saved in a table.
} 


\subsubsection{Alignment media for denatured proteins}

The possibility of measuring RDCs on denatured proteins was first demonstrated by Shortle and coworkers [177, 183]. They reported the measurement of RDCs in the denatured forms of the protein staphylococcal nuclease, a small $\alpha+\beta$ protein of 149 amino acids, oriented in either strained polyacrylamide gels [32, 184], a bicelle medium, and a liquid crystalline phase formed by a cationic lipid: cetylpyridinium bromide. All the three types of media can be used at high urea concentrations. While polyacrylamide gels and bicelles produce similar alignment tensors through steric interactions, a liquid crystalline phase of cetylpyridinium bromide aligns denatured nucleases along a different set of axes, presumably through electrostatic effects.

\subsubsection{Native-like topology persistent in the denatured state}

Shortle and coworkers [177] observed a highly significant correlation among NH RDCs for individual residues between the denatured and native states of a 131-residues (residues 10 to 140) fragment system of staphylococcal nuclease. Their result suggested that a native-like spatial positioning and orientation of chain segments (topology) persists to concentrations of at least $8.0 \mathrm{M}$ urea. The RDC data demonstrated that long-range ordering can occur well before a folding protein attains a compact conformation, a conclusion not anticipated by any of the standard models of protein folding. In another study, Shortle and workers [178] measured both NH and $\mathrm{CH}$ RDCs at a low $\mathrm{pH}$, urea denatured state and the native state in order to characterize the long-range structure that persists in the unfolded form of a 70-residue protein eglin $\mathrm{C}$. When the data sets for the two different structural states were compared, a statistically significant correlation was found between the RDCs measured in the denatured and native states with a correlation coefficient of $r=0.47$ for CH RDCs and $r=0.51$ for NH RDCs. The authors claimed that the finding directly demonstrated that the denatured state of eglin $\mathrm{C}$ had a nativelike global structure. They further developed a simple simulation procedure to show that the degree variation in $(\phi, \psi)$ angles that yields a RDC correlation of $r=0.5$ was inversely dependent on the statistical segment length, ranging from $\pm 6^{\circ}$ to $\pm 30^{\circ}$ at the upper limit.

The persistence of native topology was also used by Fieber and coworkers [179] to explain the RDCs measured in the denatured state of bovine acyl-coenzyme A binding protein (ACBP) oriented in strained polyacrylamide gels. ACBP is an 86-residue protein consisting of four helices, A1, A2, A3 and A4. The data includes RDCs measured under denaturing conditions ( $\mathrm{pH} 2.3$ and $2.5 \mathrm{M} \mathrm{GuHCl}$ ), no-denaturing conditions ( $\mathrm{pH} 2.5$ and 2.8) and the native state. The data were analyzed using the molecular fragment replacement (MFR) program [24] (Section 5.2.3), which demonstrated alpha-helix propensity in four isolated stretches along the protein backbone of ACBP, and these four stretches coincide with the location of the four native helices. This is in agreement with the findings based on secondary chemical shift values. The authors further showed that replacement of the hydrophobic side-chain of residue Ile27 with alanine in helix A2 leads to large decreases of RDCs in residues that form helix A4 in the native state. The results suggest that the Ile to Ala mutation changes the probability for the formation of long-range interactions, which are present in the acid-denatured state of the wild-type ACBP. These long-range interactions are similar to those proposed to form in the transition state of folding of ACBP. The application of RDCs in combination with a comparative mutation study thus demonstrated the presence of precursors to the folding transition state under acid-unfolding conditions. The NH RDCs were measured from spin-state-selective $\left(\mathrm{S}^{3} \mathrm{CT}\right.$ ) filtered ${ }^{1} \mathrm{H}_{-}{ }^{15} \mathrm{~N}-\mathrm{HSQC}$ experiments [41, 55] (Sections 2.2 and 3.1), while $\mathrm{C}_{\alpha}-\mathrm{C}^{\prime}, \mathrm{C}^{\prime}{ }^{15} \mathrm{~N}$, and $\mathrm{C}_{\alpha^{-}}{ }^{1} \mathrm{H}_{\alpha}$ dipolar couplings were measured from 3D HNCO-based experiments [97, 84] (Sections 3.2 and 3.7). The RDCs were extracted by frequency-displacement method.

\subsubsection{No native-like topology in the denatured state}

Louhivuori and coworkers [180] try to explain, theoretically, the characteristics of the RDCs on the denatured state using random-walk on two-dimensional (2D) plane with reflection walls. The reflection here models 
the effects of aligning medium on the motion of the dissolved proteins. A 2D random-walk model is used since exact expressions for various probabilities are readily available in $2 \mathrm{D}$, while a $3 \mathrm{D}$ random-walk is much more complicated [185]. The denatured structure was modeled as a random-flight chain [175]. They applied the probability distributions for a 2D random-walk with reflection walls [186] to calculate RDCs and residual chemical shift anisotropy measured on the denatured proteins. Their calculations yield chain length and residue position-dependent values of RDCs which is in good agreement with simulations. Thus, it is not necessary to use native-like topology to explain the RDCs measured on the denatured state. However, the proposed model is rather crude since it may introduce errors to describe the alignment in 3D solution with a $2 \mathrm{D}$ random-walk with reflecting walls. Errors may also be introduced in their model by using a homopolymer to represent a heteropolymer such as a protein in solution.

Ding and coworkers [181] as well as Mohana-Borges and coworkers [182], also questioned, experimentally, the hypothesis that the RDCs in the denatured state must be interpreted using similarity to the native state. Ding and coworkers [181] studied a destabilized mutant of the B1 domain of protein G (GB1), a 56-residue small protein consisting of four $\alpha$-helices and a $\beta$-sheet. They systematically investigated the equilibrium unfolding by recording backbone NH RDCs, the tryptophan $\mathrm{N}_{\epsilon}-\mathrm{H}$ resonance and the amide nitrogen transverse relaxation rates $(R 2 s)$ for varying $\mathrm{pH}$ values and different temperatures. The NH RDCs were recorded with a sensitivity-enhanced E. COSY method [79] (Section 3.1) and extracted by frequency-displacement. The backbone NH RDCs indicated that prior to complete unfolding, two melting hot spots were formed at the turn around T11, L12 and K13 and the N terminus of the helix at A24 and T25. The RDCs for the low pH, thermally unfolded state of GB1 were very small and did not indicate the presence of any native-like structure. Amide nitrogen transverse relaxation rates for GB1 in the folded state at different temperatures exhibited large contributions from exchange processes and the associated dynamics displayed considerable heterogeneity. After a careful analysis of the RDC and NMR relaxation data they believed that the data provided clear evidence for intermediate conformations and multi-state equilibrium unfolding and folding for this GB1 variant.

Mohana-Borges and coworkers studied the conformational propensities of the unfolded states of apomyoglobin by measurement of NH RDCs. Weak alignment of apomyoglobin in acid and urea-unfolded states was induced with both stretched and compressed polyacrylamide gels. For the acid-unfolded states, NH RDCs were measured in ${ }^{15} \mathrm{~N},{ }^{13} \mathrm{C}$-labeled protein using a 3D HNCO IPAP pulse program because of poor ${ }^{1} \mathrm{H}$ resonance dispersion. The RDCs were extracted by frequency-displacement. In $8 \mathrm{M}$ urea solution at $\mathrm{pH} 2.3$, conditions under which apomyoglobin contains no detectable secondary or tertiary structure, significant RDCs of uniform sign were observed for all residues. At pH 2.3 in the absence of urea, a change in the magnitude and/or sign of the RDCs occurs in local regions of the polypeptide where there is a high propensity for helical secondary structure. These results are interpreted on the basis of the statistical properties of the unfolded polypeptide chain, viewed as a polymer of statistical segments. For a folded protein, the magnitude and sign of the RDCs depend on the orientation of each bond vector relative to the alignment tensor of the entire molecule, which reorients as a single entity. For unfolded proteins, there is no global alignment tensor; instead, RDCs are attributed to alignment of the statistical segments or of transient elements of secondary structure. For apomyoglobin in $8 \mathrm{M}$ urea, the backbone is highly extended, with $\phi$ and $\psi$ dihedral angles favoring the $\beta$ or P(II) regions. Each statistical segment has a highly anisotropic shape, with the $\mathrm{N}-\mathrm{H}$ bond vectors approximately perpendicular to the long axis, and becomes weakly aligned in the anisotropic environment of the strained acrylamide gels. Local regions of enhanced flexibility or chain compaction are characterized by a decrease in the magnitude of the RDCs. The formation of a small population of helical structure in the acid-denatured state of apomyoglobin leads to a change in sign of the RDCs in local regions of the polypeptide; the population of helix estimated from the RDCs is in excellent agreement with that determined from chemical shifts. They further claimed that the above alignment model for the denatured apomyoglobin can also explain the patterns of RDCs reported previously for the denatured states of staphylococcal nuclease and all other denatured pro- 
teins.

Sosnick and coworkers [174] generated an unfolded state ensemble using a self-avoiding random-walk based-statistical coil model using the frequencies for the backbone $(\phi, \psi)$ angles in a coil library, a subset of the PDB. The model reproduces two apparently contradicting behaviors observed in the chemically denatured state for a variety of proteins, random coil scaling of the radius of gyration and the presence of significant amounts of local backbone structure as indicated by the existence of RDCs in the denatured state. The most stretched members of their unfolded ensemble dominate the RDC signal, whereas the uniformity of the sign of the couplings follows from the preponderance of polyproline II and $\beta$ conformers in the coil library. Agreement with the NMR data substantially improved when the backbone conformational preferences included correlations arising from the chemical and conformational identity of neighboring residues. Although the unfolded ensembles match the experimental observables, they do not display evidence of nativelike topology. One concern about their model is that the ensemble for the denatured state was generated using, exclusively, the $(\phi, \psi)$ angles appeared in the PDB where only the structures of native states are present.

\subsection{Protein-ligand interaction}

Ligand-protein interaction plays key roles in many biological processes and has been the focus of structurebased drug design. Solution NMR spectroscopy can be used to investigate such interactions. Furthermore, even when a crystal structure of the ligand-protein complex is available, NMR is still very useful for providing complementary dynamical information in solution. It is well-documented that both the bound ligand and the binding site on the protein are rather dynamical in solution, at least for most ligand-protein systems $[187,188]$. Previously, chemical shift (CS) perturbation, intermolecular NOEs, transfer NOEs in particular, and possibly relaxation measurement are the main techniques for quantifying ligand-protein interactions by NMR. More recently, RDCs measured on the bound ligand have been applied to characterize the ligand-protein interactions. In combination with other methods such as SAR by NMR, RDCs can potentially become a tool for high-throughput screening for ligands that bind the target proteins.

Tian and coworkers [189] have used RDCs to investigate the conformation of trimannoside and the differential motion at the glycosidic linkages. Each of the three pyranose rings that make up trimannoside was considered a separate rigid molecular fragment. In the structure of trimannoside, ring III is situated between ring I and ring II. Using the RDCs to derive the generalized degree of order, which in turn is used to assess the extent of motion experienced by a rigid fragment, it was shown that internal motion between rings III and I is restricted but that inter-ring motion between rings III and rings II is more significant. As a result, the relative orientation of rings III and I can be determined by superimposing their alignment tensors. Doing the same for rings III and II is not possible, as it would result in a motionally averaged orientation because the alignment tensors are not equivalent. Thus, they concluded that there exist differential motions among the three rings.

Koenig and coworkers [190] measured both NH and CH RDCs (including both backbone and sidechain $\mathrm{CH}$ RDCs) of a ligand (an undecapeptide analog) that is in fast exchange between a free state and a state where it is bound to a macroscopically ordered membrane protein (MII intermediate photoreceptor rhodopsin). RDCs were used to determine the peptide orientation with respect to the membrane by XPLOR/CNS. Furthermore, RDCs in combination with NOE distance restraints obtained from transfer NOE experiments were input into the XPLOR/CNS program to determine the structure of the bound peptide. The approximate distance restraints of the bound conformation were derived from transferred NOEs, as measured from the difference of NOESY spectra recorded prior to and after photo-activation. However, it is rather tricky to interpret the RDCs measured on a ligand that is in fast exchange between a free state and a bound state. Several dynamic processes occurred simultaneously: the tumbling of membrane with respect to the external magnetic field, the motion of ligand in the bound state, and the ligand-exchange between the free and bound forms. The authors showed that with 
a simplifying assumption it is possible to use a uniform scaling factor to interpret all the RDCs, and use the interpreted RDCs in combination with NOEs to compute the structures of the bound ligand.

Bolon and coworkers [191] have used RDCs to determine the average orientation of $\alpha$-methyl mannose bound to mannose binding-protein, and Umemoto and coworkers [192] have examined the lectin protein Galectin-3 in the absence and presence of the disaccharide LacNAc by measuring NH RDCs of both the free and complexed forms of Galectin-3. A comparison of the RMSD values for RDCs measured on Galectin-3 in solution and for RDCs calculated from a crystal structure suggests that there may be small conformational differences between the unliganded and liganded solution structures of Galectin-3. Interested reader can refer to the original papers for the details.

\subsection{Protein-protein interaction}

Protein-protein interaction is fundamental for understanding the working of a cell as a coherent unit since it plays key roles in many biological processes such as signal transduction pathways, metabolic pathways and genetic regulatory networks. At heart of these biochemical activities is the problem of molecular recognition, the physical basis for the specificity in individual pathways and in communication between different pathways and the regulatory networks. In particular, the details of protein-protein interaction are the basis for understanding why receptors interact with particular targets, and how the proteins of one pathway can be insulated from related signaling components of the other pathways, and for the development of drugs specifically targeting the interfaces of protein-protein interactions. Current experimental techniques for studying protein-protein interaction at atomic detail are mainly x-ray diffraction and NMR spectroscopy. However, it is rather difficult to crystallize a protein-protein complex due to the transient nature of many protein-protein interactions. Furthermore, crystal packing may have larger effects on the interface between two proteins than on the core of the individual proteins. Studying protein-protein interaction using NMR is challenging too. First, the complex may be too large to be characterized in detail by NMR. Second, the intermolecular NOEs are in general weak, again due to the low $\mathrm{S} / \mathrm{N}$ ratio caused by the size of the system and the inherent dynamical nature of the protein-protein interaction. However, helped by TROSY techniques, RDC alone or in combination with other NMR parameters such as chemical shift (CS) perturbation and intermolecular NOEs have been increasingly used to characterize the interaction. The details of the interactions obtained from NMR depend on the amount of experimental data: ranging from the relative orientation between two proteins by RDC alone, to the atomic detail of the interface by RDCs in combination with either CS perturbation, or intermolecular NOEs or molecular force-fields with terms such as the electrostatic and VDW interactions between the two proteins. In the following, we focus on the application of RDCs to studying protein-protein interactions. Readers who are interested in other NMR techniques for mapping protein-protein interactions should refer to an excellent recent review by Zuiderweg [193].

\subsubsection{Protein-protein orientation by RDCs}

Given the structures of two proteins or domains, Fisher and coworkers [194] have developed an approach to orient them using RDCs measured on the complex by singular value decomposition (SVD). Given a structure of a protein and the experimental RDCs, an alignment tensor $\mathbf{S}$ can be computed by using SVD to minimize

the RDC RMSD, $E_{r}$, with $E_{r}=\sqrt{\sum_{i=1}^{n} \frac{\left(r_{i}^{\prime}-r_{i}\right)^{2}}{n-1}}$ where $n$ is the total number of RDCs, $r_{i}$ and $r_{i}^{\prime}$ are, respectively, the experimental RDC for residue $i$ of the protein and the RDC back-computed from the structure using the tensor $\mathbf{S}$ by Eq. (11). As shown in Eq. (11), given a structure, $r_{i}^{\prime}$ is a function of $\mathbf{S}$ so by minimizing $E_{r}, \mathbf{S}$ can be computed by SVD [153]. After the diagnolization of the alignment tensor $\mathbf{S}$ computed by SVD, the relative orientation of the two domains in a common POF can be obtained. However, there exists four fold-degeneracy in the relative orientation obtained from RDCs measured in a single aligning medium. The authors also compared the alignment tensor computed from SVD with that computed by SA as implemented in 
XPLOR/CNS $[195,93]$ where additional restraints can be added. They applied the approach to orient the domains B (residues 59 to 81) and C (residue 100-126) of Barley lectin (BL), a 126-residue protein. Comparison of principal order frames (POFs) as separately determined for each domain indicates that the two domains are not oriented as in the crystal structure of wheat germ agglutinin, a highly homologous protein (approximately $95 \%$ sequence identity to protein BL). Furthermore, differences in the alignment tensors suggest that the two domains are not statically positioned but are experiencing different reorientational dynamics.

In a study reported by Lukin and coworkers [196], the relative orientations of monomers in the tetrameric human normal adult carbonmonoxy-hemoglobin have been determined in solution at near-physiological conditions of $\mathrm{pH}$, ionic strength, and temperature using ${ }^{1} \mathrm{H}-{ }^{15} \mathrm{~N}$ RDCs measured in both bicelles and Pf1 phages by TROSY-based sequences $[3,61]$ (Section 3.5). The input individual structures are derived from x-ray diffraction. The relative orientations obtained by fitting the x-ray structures to individual types of RDCs using either an SVD [153] method or a non-linear least-squares method, were found to be a dynamic intermediate between two previously solved crystal structures, known as the R and R2 states. In combination with exchange broadening at the subunit interface, the authors concluded that a rapid equilibrium exists between different structures that presumably include the crystallographically observed states.

In an interesting paper by Zhang and Zuiderweg [197], the relative orientation of two lobes of a 44-kDa nucleotide-binding domain (NBD) of the 70-kDa heat shock cognate (Hsc70) chaperone has been determined by an SVD method using RDCs in a single aligning medium measured by TROSY techniques [77] (Section 3.1). The pulse sequence was optimized for NBD by selecting a best value for the parameter $k$. NBD allosterically regulates, by binding either ADP or ATP in a cleft between the two main lobes, the chaperoning affinity of the attached substrate-binding domain of Hsc70 and is also a center of interaction with cochaperones that couple into the allostery. The orientation of two lobes computed from RDCs in solution deviates up to $10^{\circ}$ from their positions in 14 superimposing $x$-ray structures. Additional orientational differences of subdomains within the lobes, also from the analysis of the RDC data, unveil that the Hsc70 NBD in solution exists as a flexible molecular machine that can adjust the relative positions of all of its four subdomains.

Blackledge and coworkers [198] have developed an approach for computing the diagonalized Saupe matrix $S_{y y}$ and $S_{z z}$ (what they really computed is $D_{a}$ and $R$ but they are equivalent to $S_{y y}$ and $S_{z z}$, see Eqs. 11,12 of Section 1.1) and the orientation of a POF in a molecular frame as specified by three Euler angles $(\alpha, \beta, \gamma)$. These five parameters are computed by a least-square minimization of the following target function over all $n$ couplings associated with a given domain:

$$
\chi^{2}=\sum_{n} \frac{r_{i}-r_{i}^{\prime}\left(D_{a}, D_{r}, \alpha, \beta, \gamma, \mathbf{v}_{i}\right)}{\sigma_{i}}
$$

where $r_{i}$ and $r_{i}^{\prime}\left(D_{a}, D_{r}, \alpha, \beta, \gamma, \mathbf{v}_{i}\right)$ are, respectively, the experimental RDC for internuclear vector $i$ and the RDCs back-computed from the structure, $\mathbf{v}_{i}$ is the orientation of the vector $i$ in a molecular-fixed frame, $\alpha, \beta, \gamma$ define the orientation of POF relative to the molecular-fixed frame; $\sigma_{i}$ is the uncertainty in the experimentallymeasured coupling. The minimization algorithm searches the $D_{a}, D_{r}, \alpha, \beta, \gamma$ parametric space by random variation of these parameters, using a combination of simulated annealing, temperature regulation using fuzzy logic and Levenberg-Marquardt minimization. However, this approach is less accurate and slow compared with the method based on SVD followed by diagnolization to compute the same five parameters.

\subsubsection{Protein-protein interface by RDCs in combination with other NMR restraints}

Since RDCs can only provide the information on relative orientation of two proteins, translational restraints such as distance restraints must be supplied in order to obtain the interaction interface between two proteins. 
Such distance information can be in different forms: intermolecular NOEs, transfer NOEs, paramagnetic relaxation enhancements (PREs), vdW and electrostatic interactions as well as those NMR parameters such as CS perturbation, which are complicated functions of the internuclear distances.

The details of protein-protein interaction can be obtained through the structure determination of the entire complex using RDCs in combination with other NMR restraints. For example, Clore and coworkers [199] have determined the solution structure of the first protein-protein complex of the bacterial phosphoenolpyruvate: sugar phosphotransferase system between the N-terminal domain of enzyme I (EIN) and the histidinecontaining phosphocarrier protein (HPr) using RDCs in combination with other NMR restraints. More often, RDCs in combination with other geometric restraints have been used to determine the interface between two proteins with known individual structures. Clore and coworkers [200] have developed an approach based on conjoined rigid body/torsion angle dynamics and simulated annealing for determining the relative orientations of covalently linked protein domains using RDCs. In this approach, each domain is treated as a rigid body and the relevant degrees of conformational freedom are restricted to the backbone torsion $(\phi, \psi)$ angles of the linker between the two domains. The translational restraints were provided by the requirement that the standard geometric configuration must be kept for the linker. The target function for the minimization included both the RDC term and energy terms for keeping standard configuration for the linkers. This approach was first applied to compute the relative orientation of the two-halves of a domain-swapped dimer of the HIV-inactivating protein cyanovirin- $\mathrm{N}$ [200], and later to determine the structure of the complex between the cytoplasmic A domain (IIA(Mtl)) of the mannitol transporter II(Mannitol) and the histidine-containing phosphocarrier protein (HPr) of the E.coli phosphotransferase system [201].

In a more recent application, Jain and coworkers [202] have used RDCs to characterize the relative orientation of a $95 \mathrm{kDa}$ homotrimeric complex of the acyltransferase protein, LpxA (UDP-N-acetylglucosamine acyltransferase), and acyl carrier protein (ACP). NH RDCs for perdeuterated ACP were measured by a TROSYbased method. The bound state RDCs for ACP were calculated from the following equation since the experimentally measured RDCs for ACP bound to LpxA correspond to an average between the free state and bound state of ACP.

$$
r_{b}=\frac{r_{o}-x_{f} r_{f}}{x_{b}}
$$

where $r_{b}$ is the RDC in the bound state, $r_{o}$ is the observed RDC, $x_{f}$ is the fraction of ACP in the free state, $r_{f}$ is the RDC measured in the free state and $x_{b}$ is the fraction of ACP in the bound state. $x_{f}$ and $x_{b}$ were estimated from the known value $\left(2.0 \mu \mathrm{M}^{-1}\right)$ for the $K_{m}$ of ACP to LpxA. The transverse relaxation in the bound state is ignored. The calculated bound RDCs were first used to compute the alignment tensor for ACP by SVD. With the computed alignment tensors by SVD, the program HODDOCK1.1 [203] was used to dock ACP onto LpxA using RDCs, ambiguous interaction restraints (AIRs) derived from mutagenesis data and CS perturbation data.

McCoy and coworkers have developed a method for docking two proteins with known structures using RDCs in combination with chemical shifts (CS) [204] with the goal to replace the distance derived usually from intermolecular NOEs with restraints derived from CS perturbations. The authors showed the structure built from the crystal structures of EIN and HPr in their uncomplexed form and docked with both RDCs and CS perturbations, places HPr within $2.5 \AA$ of the position determined from the mean NMR structure of the EIN-Hpr complex. The relative position of the two proteins was obtained indirectly by minimizing the difference between the observed CS perturbations with the theoretical perturbations predicated by SHIFTS 3.0 (David Case, Scripps).

Dobrodumov and coworkers [205] have used RDCs in combination with chemical shift (CS) mapping, 
biochemical knowledge about point-to-point interactions, and contact potentials to select and filter structural models of the complex generated by docking of known substructures such as individual proteins or domains. The program FTDOCK [206] is used for docking the substructures. The empirical contact potentials are between a pair of residues from the two docking substructures, the point-to-point interaction is set to be between two $\mathrm{C}_{\alpha}-\mathrm{C}_{\alpha}$ atoms obtained from biochemical data. The models from the FTDOCK are ranked by RDCs using a Q-factor, $Q$, defined as $Q=\sqrt{\frac{\sum_{i=1}^{n}\left(r_{i}^{\prime}-r_{i}\right)^{2}}{\sum_{i=1}^{n}\left(r_{i}^{\prime}-r_{a}\right)^{2}}}$ where $n$ is the total number of RDCs, $r_{i}$ and $r_{i}^{\prime}$ are, respectively, the experimental RDC for residue $i$ of the protein and the RDC back-computed from the structure using the tensor $\mathbf{S}$ computed by SVD, $r_{a}$ is the average RDC value. If $Q<0.5$ the structures from FTDOCK is then selected. The CS perturbation data were used for ranking as follows. The perturbation data are first clustered into several patches on each protein surface. Next the number of contacts between the patches from the two docking proteins are counted and the docked structures from FTDOCK are then ranked based on the number of contacts. The power of RDCs for selection was demonstrated by the determination of protein-protein complexes using the EIN/HPr complex as an example and for establishing the domain-domain orientation in a chimeric protein, the recently determined hybrid human-E.coli thioredoxin.

Nakamura and coworkers [207] has combined the distance restraints extracted from saturation transfer (SAT) experiment with RDCs to compute the protein-protein complex. To detect the region of the complex molecule responsible for the interaction between two proteins by cross saturation measurements, both ${ }^{2} \mathrm{H}$, ${ }^{15} \mathrm{~N}$-labeled and non-labeled molecules were prepared. Saturation was applied with a WURST-20 adiabatic pulse. The reduction ratios of the peak intensities in the two ${ }^{1} \mathrm{H}-{ }^{15} \mathrm{~N}$ HSQC spectra with and without cross saturation were simulated by a relaxation matrix using the proton coordinates of the protein-protein complex. The simulated ratios were then applied to the calculation of the pseudo potential energy, $E_{s}=\frac{\sum\left(\eta_{s}-\eta_{e}\right)^{2}}{2}$, where $\eta_{s}$ and $\eta_{e}$ are respectively the simulated and experimental reduction ratios. RDCs were measured by the pulse sequences as described in [50] (Sections 2.4 and 3.1). A MD/SA program was developed to compute the complex structure by combining the pseudoenergy term for SAT, $E_{s}$, as computed above, and a term for RDCs with Amber96 force field. The method was successfully applied to determine the complex between the CAD domain of mouse caspase-activated deoxyribonuclease (CAD-CD: residues 1-87) and the CAD domain of its inhibitor (ICAD-CD: residues 1-100).

A computational approach HADDOCK (High Ambiguity Driven protein-protein Docking) based on restrained MD/SA (XPLOR/CNS) has been developed by Bonvin and coworkers [203] to incorporate, systematically, RDCs and other distance restraints. HADDOCK makes use of biochemical and/or biophysical interaction data such as CS perturbation data resulting from NMR titration experiments or mutagenesis data. This information is introduced as Ambiguous Interaction Restraints (AIRs) to drive the docking process. An AIR is defined as an ambiguous distance between two residues shown to be involved in the interaction. The accuracy of the approach is demonstrated with three molecular complexes. For two of these complexes, for which both the complex and free protein structures have been solved, NMR titration data were available. Mutagenesis data were used in the last example. In all cases, the best structures generated by HADDOCK, that is, the structures with the lowest intermolecular energies, were the closest to the published structure of the respective complexes (within 2.0 Å backbone RMSD).

\section{Conclusions and future perspectives}

Since the first successful demonstration of the tunable alignment of ubiquitin in an anisotropic liquid crystal medium only eight years ago, we have witnessed a flurry of new developments in NMR pulse techniques for the measurement of an increasing number of RDCs on both backbone and sidechain internuclear vectors. The development focuses on (a) reducing spectral overlap through various spin-state selection techniques, (b) in- 
creasing the signal to noise ratio through the minimization of magnetization loss by relaxation, and (c) saving spectrometer time and improving data quality by simultaneous measurement of several kinds of RDCs in a single experiment. During the same short eight years, the applications of RDCs have expanded from the original goal of improving the quality of biomolecular structures computed using mainly NOE distance restraints, to dynamics of biomolecules, automated chemical shift resonance assignment, protein folding, ligand-protein and protein-protein interactions. However, some hurdles remain before RDC measurement and applications become a routine NMR technique accessible to non-NMR-specialized biochemists and structural biologists. It is not always easy to find the right alignment medium or conditions for some biomolecules, and thus a better understanding of the alignment mechanism will be useful. New measurement techniques for improving both the S/N ratio and spectral resolution are always desirable, especially for large-sized proteins and proteinprotein complexes. Compared to the internuclear vectors in the protein backbone region, there are not many experiments available for the RDC measurements of internuclear vectors in the sidechains. The sidechain RDCs, especially carbon-carbon RDCs, can provide indispensable information for both the structural and dynamic study of large and perdeuterated proteins. It is still tricky to interpret and apply the measured RDC values quantitatively. As shown in Eqs. (6-12) of Section 1.1, the interpretation of the measured RDCs can be complicated, especially the separation of dynamics from structural information. This is because it is not easy to obtain the necessary dynamical information by either experimental or theoretical approaches to deconvolve structural information from dynamic one. As for the application of RDCs to structure determination, it has been rather subjective as far as the quality of the computed structures is concerned. In fact, the following problem exists for NMR structural determination in general: If heuristic methods such as simulated annealing or Monte-Carlo are used to search the conformational space, the computed structures are not guaranteed to be the real global minimum; that is, these search methods may themselves introduce additional errors. Furthermore, these heuristic methods make it very tricky to correlate the precision of the computed structures with the experimental errors. Structure determination algorithms using RDCs which can overcome the above problems are highly desirable. When RDCs are applied to determine the ligand-protein or protein-protein interactions, care must be taken that the relative orientation between two biomolecules is not perturbed by the alignment. When RDCs are used to study the conformations of denatured proteins, it is critical that the alignment does not favor certain conformations over the others. A systematic investigation of the effects of alignment on the biochemical properties of the aligned biomolecules is needed for assuring the minimum perturbation in these RDC applications. In summary, we expect continuing development of better pulse techniques for RDC measurement, especially for the sidechain RDCs. With these improved techniques, we believe that RDCs will be applied to more biological systems to answer complicated questions in biochemistry and structural biology by providing quantitative structural and dynamical information, which can not be easily obtained from other current biophysical techniques.

\section{Acknowledgments}

We would like to thank Prof. G. A. Webb for asking us to write the review, and for his encouragement, support and patience. We would like to thank Prof. Erik Zuiderweg for teaching us biomolecular NMR. Lincong Wang would like to thank Prof. Bruce Donald for his support. Lincong's work is supported by National Institutes of Health (R01 GM 65982) to Prof. Bruce Donald of Computer Science Department, Dartmouth College. We would like to thank Quincy Teng for numerous discussions, Candace Seu and Mark Dizik for their reviewing the manuscript, and Yuan Chen for her encouragement.

\section{References}

[1] M. K. Rosen, K. H. Gardner, R. C. Willis, W. E. Parris, T. Pawson, and L. E. Kay. Selective methyl group protonation of perdeuterated proteins. J. Mol. Biol., 263(5):627-36, 1996.

[2] K. H. Gardner and L. E. Kay. The use of ${ }^{2} \mathrm{H}^{13} \mathrm{C},{ }^{15} \mathrm{~N}$ multidimensional NMR to study the structure and dynamics of proteins. Annu. Rev. Biophys. Biomol. Struct., 27:357-406, 1998. 
[3] K. Pervushin, R. Riek, G. Wider, and K. Wuthrich. Attenuated t 2 relaxation by mutual cancellation of dipole-dipole coupling and chemical shift anisotropy indicates an avenue to NMR structures of very large biological macromolecules in solution. Proc. Natl. Acad. Sci. U S A, 94:12366-12371, 1997.

[4] N. Tjandra and A. Bax. Direct measurement of distances and angles in biomolecules by NMR in a dilute liquid crystalline medium. Science, 278:1111-1114, 1997.

[5] J. R. Tolman, J. M. Flanagan, M. A. Kennedy, and J. H. Prestegard. Nuclear magnetic dipole interactions in field-oriented proteins: Information for structure determination in solution. Proc. Natl. Acad. Sci. USA, 92:9279-9283, 1995.

[6] R. Freeman and E. Kupce. New methods for fast multidimensional NMR. J. Biomol. NMR, 27(2):101-13, 2003.

[7] H. M. Al-Hashimi and D. J. Patel. Residual dipolar couplings: synergy between NMR and structural genomics. J. Biomol. NMR, 22(1):1-8, 2002.

[8] P. J. Lukavsky and J. D. Puglisi. Structure determination of large biological RNAs. Methods Enzymol, 394:399-416, 2005.

[9] D. MacDonald and P. Lu. Residual dipolar couplings in nucleic acid structure determination. Curr. Opin. Struct. Biol., 12(3):337-43, 2002

[10] L. Zidek, R. Stefl, and V. Sklenar. NMR methodology for the study of nucleic acids. Curr. Opin. Struct. Biol., 11(3):275-81, 2001.

[11] J. R. Tolman and H. M. Al-Hashimi. NMR studies of biomolecular dynamics and structural plasticity using residual dipolar couplings. Annual Reports on NMR Spectroscopy, 51:105-166, 2003.

[12] A. Bax. Weak alignment offers new NMR opportunities to study protein structure and dynamics. Protein. Sci., 12(1):1-16, 2003.

[13] R. S. Lipsitz and N. Tjandra. Residual dipolar couplings in NMR structure analysis. Annu. Rev. Biophys. Biomol. Struct., 33:387-413, 2004

[14] J. H. Prestegard, C. M. Bougault, and A. I. Kishore. Residual dipolar couplings in structure determination of biomolecules. Chem. Rev., 104:3519-3540, 2004.

[15] J. H. Prestegard, K. L. Mayer, H. Valafar, and G. C. Benison. Determination of protein backbone structures from residual dipolar couplings. Methods Enzymol, 394:175-209, 2005.

[16] H. Valafar, K. L. Mayer, C. M. Bougault, P. D. LeBlond, Jr. Jenney, F. E., P. S. Brereton, M. W. Adams, and J. H. Prestegard. Backbone solution structures of proteins using residual dipolar couplings: application to a novel structural genomics target. $J$. Struct. Funct. Genomics, 5(4):241-54, 2004.

[17] L. D. Landau and E. M. Lifshitz. The Classical Theory of Fields. Pergamon Press, Oxford, 1980.

[18] A. Abragam. The Principles of Nuclear Magnetism. Clarendon Press, Oxford, 1961.

[19] L. Wang, A. V. Kurochkin, and E. R. P. Zuiderweg. An iterative fitting procedure for the determination of longitudinal NMR cross-correlation rates. J. Magn. Reson., 144:175-185, 2000.

[20] A. Saupe. Recent results in the field of liquid crystals. Angew. Chem., 7:97-112, 1968.

[21] J. Meiler, J. J. Prompers, W. Peti, C. Griesinger, and R. Bruschweiler. Model-free approach to the dynamic interpretation of residual dipolar couplings in globular proteins. J. Am. Chem. Soc., 123:6098-6107, 2001.

[22] J. R. Tolman. A novel approach to the retrieval of structural and dynamic information from residual dipolar couplings using several oriented media in biomolecular nmr spectroscopy. J. Am. Chem. Soc., 124:12020-12030, 2002.

[23] W. Peti, J. Meiler, R. Bruschweiler, and C. Griesinger. Model-free analysis of protein backbone motion from residual dipolar couplings. J. Am. Chem. Soc., 124:5822-5833, 2002.

[24] F. Delaglio, G. Kontaxis, and A. Bax. Protein structure determination using molecular fragment replacement and NMR dipolar couplings. J. Am. Chem. Soc., 122(9):2142-2143, 2000.

[25] L. Wang and B. R. Donald. Exact solutions for internuclear vectors and backbone dihedral angles from NH residual dipolar couplings in two media, and their application in a systematic search algorithm for determining protein backbone structure. $J$. Biomol. NMR, 29:223-242, 2004.

[26] L. G. Barrientos, C. Dolan, and A. M. Gronenborn. Characterization of surfactant liquid crystal phases suitable for molecular alignment and measurement of dipolar couplings. J. Biomol. NMR, 16:329-337, 2000.

[27] R. S. Prosser, J. A. Losonczi, and I. V. Shiyanovskaya. Use of a novel aqueous liquid crystalline medium for high-resolution nmr of macromolecules in solution. J. Am. Chem. Soc., 120(42):110010-11011, 1998.

[28] G. M. Clore, M. R. Starich, and A. M. Gronenborn. Measurement of residual dipolar couplings of macromolecules aligned in the nematic phase of a colloidal suspension of rod-shaped viruses. J. Am. Chem. Soc., 120:10571-10572, 1998. 
[29] M. R. Hansen, L. Mueller, and A. Pardi. Tunable alignment of macromolecules by filamentous phage yields dipolar coupling interactions. Nat. Struct. Biol, 5(12):1065-74, 1998.

[30] B. W. Koenig, J. S. Hu, M. Ottiger, S. Bose, R. W. Hendler, and A. Bax. NMR measurement of dipolar couplings in proteins aligned by transient binding to purple membrane fragments. J. Am. Chem. Soc., 121:1385-1386, 1999.

[31] K. Fleming, D. G. Gray, S. Prasannan, and S. Matthews. Cellulose crystallites: A new and robust liquid crystalline medium for the measurement of residual dipolar couplings. J. Am. Chem. Soc., 122:5224-5225, 2000.

[32] R. Tycko, F. J. Blanco, and Y. Ishii. Alignment of biopolymers in strained gels: A new way to create detectable dipole-dipole couplings in high-resolution biomolecular NMR. J. Am. Chem. Soc., 122:9340-9341, 2000.

[33] M. Ruckert and G. Otting. Alignment of biological macromolecules in novel nonionic liquid crystalline media for NMR experiments. J. Am. Chem. Soc., 122:7793-7797, 2000.

[34] S. Meier, D. Haussinger, and S. Grzesiek. Charged acrylamide copolymer gels as media for weak alignment. J. Biomol. NMR, 24(4):351-6, 2002.

[35] J. F. Trempe, F. G. Morin, Z. Xia, R. H. Marchessault, and K. Gehring. Characterization of polyacrylamide-stabilized Pfl phage liquid crystals for protein NMR spectroscopy. J. Biomol. NMR, 22(1):83-7, 2002.

[36] A. Bax, G. Kontaxis, and N. Tjandra. Dipolar couplings in macromolecular structure determination. Methods Enzymol, 339:127-174, 2001.

[37] K. Fleming and S. Matthews. Media for studies of partially aligned states. Methods Mol. Biol., 278:79-88, 2004.

[38] M. Ottiger, F. Delaglio, and A. Bax. Measurement of J and dipolar couplings from simplified two-dimensional NMR spectra. J. Magn. Reson., 131(2):373-8, 1998.

[39] A. Meissner, J. O. Duus, and O. W. Sorensen. Integration of spin-state-selective excitation into 2D NMR correlation experiments with the heteronuclear ZQ/2Q $\pi$ rotations for $1 \mathrm{JXH}$ - resolved E.COSY-type measurements of heteronuclear coupling constants in proteins. J. Biomol. NMR, 10(1):89-94, 1997.

[40] A. Meissner, J. O. Duus, and O. W. Sorensen. Spin-state-selective excitation. application for E.COSY-type measurement of jhh coupling constants. J. Magn. Reson., 128(1):92-97, 1997.

[41] M. D. Sorensen, A. Meissner, and O.W. Sorensen. Spin-state-selective coherence transfer via intermediate states of two-spin coherence in IS spin systems: Application to E.COSY-type measurement of j coupling constants. J. Biomol. NMR, 10:181-186, 1997.

[42] C. Griesinger, O. W. Sorensen, and R. R. Ernst. Two-dimensional correlation of connected NMR transitions. J. Am. Chem. Soc., 107:6394-6396, 1985.

[43] N. Tjandra and A. Bax. Large variations in 13C chemical shift anisotropy in proteins correlate with secondary structure. J. Am. Chem. Soc., 119(40):9576-9577, 1997.

[44] W. Hu, Z. Zhang, and Y. Chen. A high sensitivity 3D experiment for measuring ${ }^{13} \mathrm{C}_{\alpha}-{ }^{1} \mathrm{H}_{\alpha}$ residual dipolar coupling constants. J. Magn. Reson., 165(2):248-52, 2003.

[45] P. Andersson, J. Weigelt, and G. Otting. Spin-state selection filters for the measurement of heteronuclear one-bond coupling constants. J. Biomol. NMR, 12:435-441, 1998.

[46] M. D. Sorensen, A. Meissner, and O. W. Sorensen. ${ }^{13} \mathrm{C}$ natural abundance S3E and S3CT experiments for measurement of J coupling constants between ${ }^{13} \mathrm{C}_{\alpha}$ or ${ }^{1} \mathrm{H}_{\alpha}$ and other protons in a protein. J. Magn. Reson., pages 237-242, 1999.

[47] B. Brutscher. Accurate measurement of small spin-spin couplings in partially aligned molecules using a novel J-mismatch compensated spin-state-selection filter. J. Magn. Reson., 151:332-338, 2001.

[48] G. T. Montelione and G. Wagner. Accurate measurements of homonuclear $\mathrm{HN}-\mathrm{H}_{\alpha}$ coupling constants in polypeptides using heteronuclear 2D NMR experiments. J. Am. Chem. Soc., 111(14):5474-5475, 1989.

[49] A. Bax, G. W. Vuister, S. Grzesiek, F. Delaglio, A. C. Wang, R. Tschudin, and G. Zhu. Measurement of homo- and heteronuclear J couplings from quantitative J correlation. Methods Enzymol, 239:79-105, 1994.

[50] N. Tjandra, S. Grzesiek, , and A. Bax. Magnetic field dependence of nitrogen-proton j splittings in ${ }^{15} \mathrm{~N}$-enriched human ubiquitin resulting from relaxation interference and residual dipolar coupling. J. Am. Chem. Soc., 118:6264-6272, 1997.

[51] J. R. Tolman and J. H. Prestegard. A quantitative J-correlation experiment for the accurate measurement of one-bond amide 15N-1H couplings in proteins. J. Magn. Reson., 112:245-252, 1996.

[52] G. W. Vuister and A. Bax. Quantitative J correlation: a new approach for measuring homonuclear three-bond j(hnh.alpha.) coupling constants in ${ }^{15} \mathrm{~N}$-enriched proteins. J. Am. Chem. Soc., 115(17):7772-7777, 1993.

[53] K. Pervushin, G. Wider, and K. Wuthrich. Single transition-to-single transition polarization transfer (st2-pt) in $\left[{ }^{15} \mathrm{~N},{ }^{1} \mathrm{H}\right]-$ TROSY. J. Biomol. NMR, 12:345-348, 1998. 
[54] K. Pervushin. Impact of transverse relaxation optimized spectroscopy (trosy) on NMR as a technique in structural biology. $Q$. Rev. Biophys., 33(2):161-97, 2000.

[55] M. H. Lerche, A. Meissner, F. M. Poulsen, and O. W. Sorensen. Pulse sequences for measurement of one-bond ${ }^{15} \mathrm{~N}-{ }^{1} \mathrm{H}$ coupling constants in the protein backbone. J. Magn. Reson., 140:259-263, 1999.

[56] J. H. Prestegard, H. M. al Hashimi, and J. R. Tolman. NMR structures of biomolecules using field oriented media and residual dipolar couplings. Q. Rev. Biophys., 33(4):371-424, 2000.

[57] J. H. Prestegard, J. R. Tolman, H. M. al Hashimi, and M. Andrec. Protein structure and dynamics from field-induced residual dipolar couplings. Biological Magnetic Resonance, 17:311-355, 1999.

[58] J. R. Tolman and J. H. Prestegard. Measurement of amide ${ }^{15} \mathrm{~N}-{ }^{1} \mathrm{H}$ one-bond couplings in proteins using accordion heteronuclear-shift-correlation experiments. J. Magn. Reson. B, 112(3):269-74, 1996.

[59] A. Bax and N. Tjandra. High-resolution heteronuclear NMR of human ubiquitin in an aqueous liquid crystalline medium. $J$. Biomol. NMR, 10:289-292, 1997.

[60] B. Cutting, J. R. Tolman, S. Nanchen, and G. Bodenhausen. Accurate measurement of residual dipolar couplings in anisotropic phase. J. Biomol. NMR, 23:195-200, 2002.

[61] G. Kontaxis, G. M. Clore, and A. Bax. Evaluation of cross-correlation effects and measurement of one-bond couplings in proteins with short transverse relaxation times. J. Magn. Reson., 143:184-196, 2000.

[62] J. P. Marino, J. L. Diener, P. B. Moore, and C. Griesinger. Multiple-quantum coherence dramatically enhances the sensitivity of $\mathrm{CH}$ and $\mathrm{CH} 2$ correlations in uniformly ${ }^{13} \mathrm{C}$-labeled rna. J. Am. Chem. Soc., 119:7361-7366, 1995.

[63] A. G. Cavanagh, J. Palmer III, P. E. Wright, and M. Rance. Sensitivity improvement in proton-detected two-dimensional heteronuclear relay spectroscopy. J. Magn. Reson., 91:429-436, 1991.

[64] L. E. Kay, P Keifer, and T Saarinen. Pure absorption gradient enhanced heteronuclear single quantum correlation spectroscopy with improved sensitivity. J. AM. Chem. Soc., 114:10663-10665, 1992.

[65] B. Luy and J. P. Marino. JE-TROSY: combined J- and TROSY-spectroscopy for the measurement of one-bond couplings in macromolecules. J. Magn. Reson., 163(1):92-8, 2003.

[66] J. Boisbouvier, F. Delaglio, and A. Bax. Direct observation of dipolar couplings between distant protons in weakly aligned nucleic acids. Proc. Natl. Acad. Sci. U S A, 100:11333-11338, 2003.

[67] C. W. Vander Kooi, E. Kupce, E. R. Zuiderweg, and M. Pellecchia. Line narrowing in spectra of proteins dissolved in a dilute liquid crystalline phase by band-selective adiabatic decoupling: application to $1 \mathrm{hn}-15 \mathrm{n}$ residual dipolar coupling measurements. J. Biomol. NMR, 15(4):335-8, 1999.

[68] K. E. Kover and G. Batta. More line narrowing in TROSY by decoupling of long-range couplings: shift correlation and $1 \mathrm{JNC}^{\prime}$ coupling constant measurements. J. Magn. Reson., 170(2):184-90, 2004.

[69] K. Pervushin and B. Vogeli. Observation of individual transitions in magnetically equivalent spin systems. J. Am. Chem. Soc., 125(32):9566-7, 2003.

[70] K. Pervushin, B. Vogeli, T. N. Heinz, and P. H. Hunenberger. Measuring ${ }^{1} \mathrm{H}^{1}{ }^{1} \mathrm{H}$ and ${ }^{1} \mathrm{H}^{-}{ }^{13} \mathrm{C}$ RDCs in methyl groups: example of pulse sequences with numerically optimized coherence transfer schemes. J. Magn. Reson., 172(1):36-47, 2005.

[71] P. Permi and A. Annila. Transverse relaxation optimised spin-state selective NMR experiments for measurement of residual dipolar couplings. J. Biomol. NMR, 16(3):221-7, 2000.

[72] R. A. Engh and R. Huber. Accurate bond and angle parameters for x-ray protein structure refinement. Acta Cryst., A47:392400, 1991.

[73] S. Vijay-Kumar, C. E. Bugg, and W. J. Cook. Structure of ubiquitin refined at 1.8 Å resolution. J. Mol. Biol., 194:531-544, 1987.

[74] Kurt Wüthrich. NMR of Proteins and Nucleic Acids. John Wiley \& Sons, 1986.

[75] A. Kaikkonen and G. Otting. Residual dipolar ${ }^{1} \mathrm{H}-{ }^{1} \mathrm{H}$ couplings of methyl groups in weakly aligned proteins. J. Am. Chem. Soc., 123(8):1770-1, 2001.

[76] F. Cordier, A. J. Dingley, and S. Grzesiek. A doublet-separated sensitivity-enhanced HSQC for the determination of scalar and dipolar one-bond J-couplings. J. Biomol. NMR, 13:175-180, 1999.

[77] D. Yang, R. A. Venters, G. A. Mueller, W. Y. Choy, and L. E. Kay. TROSY-based HNCO pulse sequences for the measurement of $1 \mathrm{HN}-15 \mathrm{~N}, 15 \mathrm{~N}-13 \mathrm{CO}, 1 \mathrm{HN}-13 \mathrm{CO}, 13 \mathrm{CO}-13 \mathrm{C}$ (alpha) and $1 \mathrm{HN}-13 \mathrm{C}$ (alpha) dipolar couplings in 15N, 13C, 2H-labeled proteins. J. Biomol. NMR., 14:333-343, 1999.

[78] K. Ding and A. M. Gronenborn. Sensitivity-enhanced E.COSY-type HSQC experiments for accurate measurements of onebond ${ }^{15} \mathrm{~N}-{ }^{1} \mathrm{H}_{\mathrm{N}}$ and ${ }^{15} \mathrm{~N}-{ }^{13} \mathrm{C}^{\prime}$ and two-bond ${ }^{13} \mathrm{C}^{\prime}-{ }^{1} \mathrm{H}_{\mathrm{N}}$ residual dipolar couplings in proteins. J. Magn. Reson., 158(1-2):173-7, 2002. 
[79] K. Ding and A. M. Gronenborn. Sensitivity-enhanced 2D IPAP, TROSY-anti-TROSY, and E.COSY experiments: alternatives for measuring dipolar 15N-1HN couplings. J. Magn. Reson., 163:208-214, 2003.

[80] R. L. McFeeters, C. A. Fowler, V. V. Gaponenko, and R. A. Byrd. Efficient and precise measurement of ${ }^{1} \mathrm{H}_{\alpha}-{ }^{13} \mathrm{C}_{\alpha}, \mathrm{C}_{\alpha}-\mathrm{C}^{\prime}$, $\mathrm{C}_{\alpha}-\mathrm{C}_{\beta}$ and $\mathrm{H}(\mathrm{N})-\mathrm{N}$ residual dipolar couplings from 2D H(N)-N correlation spectra. J. Biomol. NMR, 31(1):35-47, 2005.

[81] P. Permi. Two simple nmr experiments for measuring dipolar couplings in asparagine and glutamine side chains. J. Magn. Reson., 153(2):267-72, 2001.

[82] L. Wang and B. R. Donald. Analysis of a systematic search-based algorithm for determining protein backbone structure from a minimal number of residual dipolar couplings. In IEEE Computer Society Bioinformatics Conference, pages 319-330, Stanford University, CA, 2004.

[83] N. Tjandra and A. Bax. Measurement of dipolar contributions to ${ }^{1} \mathrm{~J}_{\mathrm{C}_{\alpha}} \mathrm{H}_{\alpha}$ splittings from magnetic-field dependence of $\mathbf{J}$ modulation in two-dimensional nmr spectra. J. Magn. Reson., 124(2):512-5, 1997.

[84] D. Yang, J. R. Tolman, N. K. Goto, and L. E. Kay. An HNCO-based pulse scheme for the measurement of, 13C(alpha)$1 \mathrm{H}$ (alpha) one-bond dipolar couplings in ${ }^{15} \mathrm{~N},{ }^{13} \mathrm{C}$ labeled proteins. J. Biomol. NMR, 12:325-332, 1998.

[85] A. Bax and M. Ikura. An efficient 3D NMR technique for correlating the proton and ${ }^{15} \mathrm{~N}$ backbone amide resonances with the alpha-carbon of the preceding residue in uniformly ${ }^{15} \mathrm{~N} /{ }^{13} \mathrm{C}$ enriched proteins. J. Biomol. NMR, 1:99-104, 1991.

[86] T. K. Hitchens, S. A. McCallum, and G. S. Rule. A J(CH)-modulated 2D (HACACO)NH pulse scheme for quantitative measurement of ${ }^{13} \mathrm{C}_{\alpha}-{ }^{1} \mathrm{H}_{\alpha}$ couplings in (15)n, (13)c-labeled proteins. J. Magn. Reson., 140(1):281-4, 1999.

[87] P. Wurtz, K. Fredriksson, and P. Permi. A set of HA-detected experiments for measuring scalar and residual dipolar couplings. J. Biomol. NMR, 31(4):321-30, 2005.

[88] T. Parella and J. Belloc. Spin-state-selective excitation in selective 1D inverse NMR experiments. J. Magn. Reson., 148(1):7887, 2001.

[89] M. Ottiger and A. Bax. Determination of relative N-HN, N-C', C-C', and C-H effective bond lengths in a protein by NMR in a dilute liquid crystalline phase. J. Am. Chem. Soc., 120(2):12334-12341, 1998.

[90] A. W. Giesen, L. C. Bae, C. L. Barrett, J. A. Chyba, M. M. Chaykovsky, M. C. Cheng, J. H. Murray, E. J. Oliver, S. M. Sullivan, J. M. Brown, and S. W. Homans. ${ }^{1} \mathrm{H}$-filtered correlation experiments for assignment and determination of coupling constants in backbone labelled proteins. J. Biomol. NMR, 22(1):21-6, 2002.

[91] P. Permi, T. Sorsa, I. I. Kilpelainen, and A. Annila. $\mathrm{Hn}\left(\alpha / \beta\right.$-coca-j) experiment for measurement of ${ }^{1} \mathrm{~J}_{\mathrm{C}^{\prime} \mathrm{C}_{\alpha}}$ couplings from two-dimensional. J. Magn. Reson., 141(1):44-51, 1999.

[92] M. Salzmann, K. Pervushin, G. Wider, H. Senn, and K. Wuthrich. Trosy in triple-resonance experiments: new perspectives for sequential NMR assignment of large proteins. Proc. Natl. Acad. Sci. U S A, 95(23):13585-90, 1998.

[93] G. M. Clore, A. M. Gronenborn, and N. Tjandra. Direct structure refinement against residual dipolar couplings in the presence of rhombicity of unknown magnitude. J. Magn. Reson., 131:159-162, 1998.

[94] G. M. Clore, A. M. Gronenborn, and A. Bax. A robust method for determining the magnitude of the fully asymmetric alignment tensor of oriented macromolecules in the absence of structural information. J. Magn. Reson., 133:216-221, 1998.

[95] J. Evenas, A. Mittermaier, D. Yang, and L. E. Kay. Measurement of ${ }^{13} \mathrm{C}_{\alpha}-{ }^{13} \mathrm{C}_{\beta}$ dipolar couplings in ${ }^{15} \mathrm{~N},{ }^{13} \mathrm{C},{ }^{2} \mathrm{H}-\mathrm{labeled}$ proteins: application to domain orientation in maltose binding protein. J. Am. Chem. Soc., 123(12):2858-64, 2001.

[96] A. Mittermaier and L. E. Kay. $\chi_{1}$ torsion angle dynamics in proteins from dipolar couplings. J. Am. Chem. Soc., 123(28):6892903, 2001.

[97] P. Permi, P. R. Rosevear, and A. Annila. A set of HNCO-based experiments for measurement of residual dipolar couplings in 15N, 13C,(2H)-labeled proteins. J. Biomol. NMR, 17:43-54, 2000.

[98] P. Permi, S. Heikkinen, I. Kilpelainen, and A. Annila. Measurement of ${ }^{1} \mathrm{~J}_{\mathrm{NC}^{\prime}}$ and ${ }^{2} \mathrm{~J}_{\mathrm{H}_{\mathrm{N}} \mathrm{C}^{\prime}}$ couplings from spin-state-selective two-dimensional correlation spectrum. J. Magn. Reson., 140(1):32-40, 1999.

[99] J. J. Chou, F. Delaglio, and A. Bax. Measurement of one-bond ${ }^{15} \mathrm{~N}^{13} \mathrm{C}^{\prime}$ dipolar couplings in medium sized proteins. J. Biomol. NMR, 18:101-105, 2000.

[100] N. Tjandra, J. Marquardt, and G. M. Clore. Direct refinement against proton-proton dipolar couplings in NMR structure determination of macromolecules. J. Magn. Reson., 142(2):393-6, 2000.

[101] G. Otting, M. Ruckert, M. H. Levitt, and A. Moshref. NMR experiments for the sign determination of homonuclear scalar and residual dipolar couplings. J. Biomol. NMR, 16(4):343-6, 2000.

[102] W. Peti and C. Griesinger. Measurement of magnitude and sign of H,H-dipolar couplings in proteins. J. Am. Chem. Soc., 122(16):3975-3976, 2000.

[103] M. Cai, H. Wang, E. T. Olejniczak, R. P. Meadows, A. H. Gunasekera, N. Xu, and S. W. Fesik. Accurate measurement of H(N)-H(alpha) residual dipolar couplings in proteins. J. Magn. Reson., 139:451-453, 1999. 
[104] F. Tian, C. A. Fowler, E. R. Zartler, Jr. Jenney, F. A., M. W. Adams, and J. H. Prestegard. Direct measurement of ${ }^{1} \mathrm{H}^{1}{ }^{1} \mathrm{H}$ dipolar couplings in proteins: a complement to traditional NOE measurements. J. Biomol. NMR, 18(1):23-31, 2000.

[105] F. Tian, P. J. Bolon, and J. H. Prestegard. Intensity-based measurement of homonuclear residual dipolar couplings from CTCOSY. J. Am. Chem. Soc., 121(33):7712-7713, 1999.

[106] Z. Wu and A. Bax. Measurement of long-range ${ }^{1} \mathrm{H}^{-1} \mathrm{H}$ dipolar couplings in weakly aligned proteins. J. Am. Chem. Soc., 124(33):9672-3, 2002.

[107] N. Sibille, B. Bersch, J. Coves, M. Blackledge, and B. Brutscher. Side chain orientation from methyl ${ }^{1} \mathrm{H}-{ }^{1} \mathrm{H}$ residual dipolar couplings measured in highly deuterated proteins. J. Am. Chem. Soc., 124(49):14616-25, 2002.

[108] E. de Alba, M. Suzuki, and N. Tjandra. Simple multidimensional NMR experiments to obtain different types of one-bond dipolar couplings simultaneously. J. Biomol. NMR, 19:63-67, 2001.

[109] V. Vijayan and M. Zweckstetter. Simultaneous measurement of protein one-bond residual dipolar couplings without increased resonance overlap. J. Magn. Reson., 174(2):245-53, 2005.

[110] H. L. Wienk, M. M. Martinez, G. N. Yalloway, J. M. Schmidt, C. Perez, H. Ruterjans, and F. Lohr. Simultaneous measurement of protein one-bond and two-bond nitrogen-carbon coupling constants using an internally referenced quantitative $\mathrm{j}$-correlated [(15)N,(1)H]-TROSY-HNC experiment. J. Biomol. NMR, 25(2):133-45, 2003.

[111] J. H. Prestegard, H. Valafar, J. Glushka, and F. Tian. Nuclear magnetic resonance in the era of structural genomics. Biochemistry, 40(30):8677-85, 2001.

[112] Y. X. Wang, J. L. Marquardt, P. Wingfield, S. J. Stahl, S. Lee-Huang, D. Torchia, and A. Bax. Simultaneous measurement of $1 \mathrm{H}-15 \mathrm{~N}, 1 \mathrm{H}-13 \mathrm{C}$, and $15 \mathrm{~N}-13 \mathrm{C}$ ' dipolar couplings in a perdeuterated $30 \mathrm{kDa}$ protein dissolved in a dilute liquid crystalline phase. J. Am. Chem. Soc., 120(29):7385-7386, 1998.

[113] K. Ding and A. M. Gronenborn. Simultaneous and accurate determination of one-bond ${ }^{15} \mathrm{~N}-{ }^{13} \mathrm{C}^{\prime}$ and two-bond ${ }^{1} \mathrm{H}_{\mathrm{N}}-{ }^{13} \mathrm{C}^{\prime}$ dipolar couplings. J. Am. Chem. Soc., 125(38):11504-5, 2003.

[114] M. Ottiger, F. Delaglio, J. L. Marquardt, N. Tjandra, and A. Bax. Measurement of dipolar couplings for methylene and methyl sites in weakly oriented macromolecules and their use in structure determination. J. Magn. Reson., 134(2):365-9, 1998.

[115] M. Ottiger and A. Bax. How tetrahedral are methyl groups in proteins? a liquid crystal NMR study. J. Am. Chem. Soc., 121(19):4690-4695, 1999.

[116] D. Yang and L. E. Kay. Improved 1HN-detected triple resonance TROSY-based experiments. J. Biomol. NMR, 13:3-9, 1999.

[117] R. Weisemann, H. Ruterjans, H. Schwalbe, J. Schueler, W. Bermel, and C. Griesinger. Determination of HN, $\mathrm{H}_{\alpha}$ and HN, C' coupling constants in ${ }^{13} \mathrm{C},{ }^{15} \mathrm{~N}$-labeled proteins. J. Biomol. NMR, 4:231-240, 1994.

[118] K. Ding and A. M. Gronenborn. Protein backbone 1H(N)-13Calpha and 15N-13Calpha residual dipolar and J couplings: new constraints for NMR structure determination. J. Am. Chem. Soc., 126(20):6232-3, 2004.

[119] T. Carlomagno, W. Peti, and C. Griesinger. A new method for the simultaneous measurement of magnitude and sign of ${ }^{1} \mathrm{D}_{\mathrm{CH}}$ and ${ }^{1} \mathrm{D}_{\mathrm{HH}}$ dipolar couplings in methylene groups. J. Biomol. NMR., 17:99-109, 2000.

[120] J. J. Chou and A. Bax. Protein side-chain rotamers from dipolar couplings in a liquid crystalline phase. J. Am. Chem. Soc., 123:3844-3845, 2001.

[121] S. Grzesiek and A. Bax. Correlating backbone amide and side chain resonances in larger proteins by multiple relayed triple resonance NMR. J. Am. Chem. Soc., 114:6291-6293, 1992.

[122] S. Meier, D. Haussinger, P. Jensen, M. Rogowski, and S. Grzesiek. High-accuracy residual ${ }^{1} \mathrm{H}_{\mathrm{N}^{-}}{ }^{13} \mathrm{C}_{\text {and }}{ }^{1} \mathrm{H}_{\mathrm{N}^{-}}{ }^{1} \mathrm{H}_{\mathrm{N}}$ dipolar couplings in perdeuterated proteins. J. Am. Chem. Soc., 125(1):44-5, 2003.

[123] H. Kuboniwa, S. Grzesiek, F. Delaglio, and A. Bax. Measurement of HN-H alpha J couplings in calcium-free calmodulin using new 2D and 3D water-flip-back methods. J. Biomol. NMR, 4(6):871-8, 1994.

[124] P. Permi. Measurement of residual dipolar couplings from ${ }^{1} \mathrm{H}_{\alpha}$ to ${ }^{13} \mathrm{C}_{\alpha}$ and ${ }^{15} \mathrm{~N}$ using a simple HNCA-based experiment. $J$. Biomol. NMR, 27(4):341-9, 2003.

[125] A. Eletsky, O. Moreira, H. Kovacs, and K. Pervushin. A novel strategy for the assignment of side-chain resonances in completely deuterated large proteins using ${ }^{13} \mathrm{C}$ spectroscopy. J. Biomol. NMR, 26(2):167-79, 2003.

[126] B. Vogeli, H. Kovacs, and K. Pervushin. Measurements of side-chain ${ }^{13} \mathrm{C}-{ }^{13} \mathrm{C}$ residual dipolar couplings in uniformly deuterated proteins. J. Am. Chem. Soc., 126(8):2414-20, 2004.

[127] E. Miclet, J. Boisbouvier, and A. Bax. Measurement of eight scalar and dipolar couplings for methine-methylene pairs in proteins and nucleic acids. J. Biomol. NMR, 31(3):201-16, 2005.

[128] E. Miclet, D. C. Williams Jr, G. M. Clore, D. L. Bryce, J. Boisbouvier, and A. Bax. Relaxation-optimized NMR spectroscopy of methylene groups in proteins and nucleic acids. J. Am. Chem. Soc., 126(34):10560-70, 2004. 
[129] L. Zidek, H. Wu, J. Feigon, and V. Sklenar. Measurement of small scalar and dipolar couplings in purine and pyrimidine bases. J. Biomol. NMR, 21(2):153-60, 2001.

[130] J. Yan, T. Corpora, P. Pradhan, and J. H. Bushweller. MQ-hCN-based pulse sequences for the measurement of 13C1'-1H1', 13C1'-15N, 1H1'-15N, 13C1'-13C2', 1H1'-13C2',13C6/8-1H6/8, 13C6/8-15N, 1H6/8-15N, 13C6-13C5, 1H6-13C5 dipolar couplings in 13C, 15N-labeled DNA (and RNA). J. Biomol. NMR, 22(1):9-20, 2002.

[131] R. Fiala, F Jiang, and V. Sklenar. Sensitivity optimized $\mathrm{HCN}$ and $\mathrm{HCNCH}$ experiments for ${ }^{13} \mathrm{C} /{ }^{15} \mathrm{~N}$ labeled oligonucleotides. J. Biomol. NMR, 12(3):373-383, 1998.

[132] R. Fiala, J. Czernek, and V. Sklenar. Transverse relaxation optimized triple-resonance NMR experiments for nucleic acids. $J$. Biomol. NMR, 16(4):291-302, 2000.

[133] V. Sklenar, T. Dieckmann, S. E. Butcher, and J. Feigon. Optimization of triple-resonance HCN experiments for application to larger RNA oligonucleotides. J. Magn. Reson., 130(1):119-24, 1998.

[134] W. Hu, Y. Q. Gosser, W. Xu, and D. J. Patel. Novel 2D and 3D multiple-quantum bi-directional HCNCH experiments for the correlation of ribose and base protons/carbons in ${ }^{13} \mathrm{C} /{ }^{15} \mathrm{~N}$ labeled RNA. J. Biomol. NMR, 20(2):167-72, 2001.

[135] J. Boisbouvier, D. L. Bryce, E. O’Neil-Cabello, E. P. Nikonowicz, and A. Bax. Resolution-optimized NMR measurement of ${ }^{1} \mathrm{D}_{\mathrm{CH}},{ }^{1} \mathrm{D}_{\mathrm{CC}}$ and ${ }^{2} \mathrm{D}_{\mathrm{CH}}$ residual dipolar couplings in nucleic acid bases. J. Biomol. NMR., 30:287-301, 2004.

[136] C. P. Jaroniec, J. Boisbouvier, I. Tworowska, E. P. Nikonowicz, and A. Bax. Accurate measurement of ${ }^{15} \mathrm{~N}-{ }^{13} \mathrm{C}$ residual dipolar couplings in nucleic acids. J. Biomol. NMR, 31(3):231-41, 2005.

[137] W. Hu, S. Bouaziz, E. Skripkin, and A. Kettani. Determination of 3J(H3i, Pi+1) and 3J(H5i/5i, Pi) coupling constants in ${ }^{13}$ C-labeled nucleic acids using constant-time HMQC. J. Magn. Reson., 139(1):181-5, 1999.

[138] T. Szyperski, C. Fernandez, A. Ono, K. Wuthrich, and M. Kainosho. The $2 \mathrm{D}[31 \mathrm{P}]$ spin-echo-difference constant-time $\left[{ }^{13} \mathrm{C}\right.$, $\left.{ }^{1} \mathrm{H}\right]$-HMQC experiment for simultaneous determination of 3J(H3'P) and 3J(C4'P) in ${ }^{13} \mathrm{C}$-labeled nucleic acids and their protein complexes. J. Magn. Reson., 140(2):491-4, 1999.

[139] Z. Wu, N. Tjandra, and A. Bax. Measurement of 1H3'-31P dipolar couplings in a DNA oligonucleotide by constant-time NOESY difference spectroscopy. J. Biomol. NMR, 19(4):367-70, 2001.

[140] P. Vallurupalli and P. B. Moor. Measurement of H2'-C2' and H3'-C3' dipolar couplings in RNA molecules. J. Biomol. NMR, 24(1):63-6, 2002.

[141] C. G. Hoogstraten and A. Pardi. Measurement of carbon-phosphorus J coupling constants in RNA using spin-echo difference constant-time HCCH-COSY. J. Magn. Reson., 133(1):236-40, 1998.

[142] E. Miclet, E. O’Neil-Cabello, E. P. Nikonowicz, D. Live, and A. Bax. ${ }^{1} \mathrm{H}^{-1} \mathrm{H}$ dipolar couplings provide a unique probe of RNA backbone structure. J. Am. Chem. Soc., 125(51):15740-1, 2003.

[143] E. O’Neil-Cabello, D. L. Bryce, E. P. Nikonowicz, and A. Bax. Measurement of five dipolar couplings from a single 3D NMR multiplet applied to the study of RNA dynamics. J. Am. Chem. Soc., 126(1):66-7, 2004.

[144] C. J. Langmead and B. R. Donald. An Expectation/Maximization nuclear vector replacement algorithm for automated NMR resonance assignments. J. Biomol. NMR, 29(2):111-138, 2004.

[145] J. C. Hus, J. J. Prompers, and R. Bruschweiler. Assignment strategy for proteins with known structure. J. Magn. Reson., 157:119-123, 2002.

[146] Y. S. Jung and M. Zweckstetter. Backbone assignment of proteins with known structure using residual dipolar couplings. J. Biomol. NMR., 30:25-35, 2004.

[147] H. M. Al-Hashimi, A. Gorin, A. Majumdar, Y. Gosser, and D. J. Patel. Towards structural genomics of rna: rapid NMR resonance assignment and simultaneous RNA tertiary structure determination using residual dipolar couplings. J. Mol. Biol., 318(3):637-649, 2002.

[148] F. Tian, H. Valafar, and J. H. Prestegard. A dipolar coupling based strategy for simultaneous resonance assignment and structure determination of protein backbones. J. Am. Chem. Soc., 123(47):11791-11796, 2001.

[149] J. Meiler and D. Baker. Rapid protein fold determination using unassigned NMR data. Proc. Natl. Acad. Sci. USA, 100:1540415409, 2003.

[150] J. Meiler and D. Baker. The fumarate sensor DcuS: progress in rapid protein fold elucidation by combining protein structure prediction methods with NMR spectroscopy. J. Magn. Reson., 173:310-316, 2005.

[151] T. H. Cormen, C. E. Leiserson, R. L. Rivest, and C Stein. Introduction to Algorithms. The MIT Press, 2001.

[152] A. P. Dempster, N. M. Larid, and D. B. Rubin. Maximum likelihood from incomplete data via the EM-algorithm. J. R. Statist. Sco. B, 39:1-38, 1977.

[153] J. A. Losonczi, M. Andrec, M. W. Fischer, and J. H. Prestegard. Order matrix analysis of residual dipolar couplings using singular value decomposition. J. Magn. Reson., 138(2):334-342, 1999. 
[154] Y. S. Jung and M. Zweckstetter. Mars-robust automatic backbone assignment of proteins. J. Biomol. NMR., 30:11-23, 2004.

[155] N. Tjandra, J. G. Omichinski, A. M. Gronenborn, G. M. Clore, and A. Bax. Use of dipolar ${ }^{1} \mathrm{H}_{-}{ }^{15} \mathrm{~N}$ and ${ }^{1} \mathrm{H}^{-}{ }^{13} \mathrm{C}$ couplings in the structure determination of magnetically oriented macromolecules in solution. Nat. Struct. Biol., 4:732-738, 1997.

[156] G. M. Clore, M.R. Starich, C. A. Bewley, M. Cai, and J Kuszewski. Impact of residual dipolar couplings on the accuracy of nmr structures determined from a minimal number of noe restraints. J. Am. Chem. Soc., 121:6513-6514, 1999.

[157] A. W. Giesen, S. W. Homans, and J. M. Brown. Determination of protein global folds using backbone residual dipolar coupling and long-range NOE restraints. J. Biomol. NMR, 25:63-71, 2003.

[158] G. A. Mueller, W. Y. Choy, D. Yang, J. D. Forman-Kay, R. A. Venters, and L. E. Kay. Global folds of proteins with low densities of NOEs using residual dipolar couplings: application to the 370-residue maltodextrin-binding protein. J. Mol. Biol., 300:197-212, 2000.

[159] W. Y. Choy, M. Tollinger, G. A. Mueller, and L. E. Kay. Direct structure refinement of high molecular weight proteins against residual dipolar couplings and carbonyl chemical shift changes upon alignment: an application to maltose binding protein. $J$. Biomol. NMR, 21:31-40, 2001.

[160] J. C. Hus, D. Marion, and M. Blackledge. Determination of protein backbone using only residual dipolar couplings. J. Am. Chem. Soc., 123:1541-1542, 2001.

[161] M. Andrec, P. Du, and R. M. Levy. Protein backbone structure determination using only residual dipolar couplings from one ordering medium. J. Biomol. NMR, 21(4):335-347, 2001.

[162] C. A. Rohl and D. Baker. De Novo determination of protein backbone structure from residual dipolar couplings using Rosetta. J. Am. Chem. Soc., 124(11):2723-2729, 2002.

[163] W. J. Wedemeyer, C. A. Rohl, and H. A. Scheraga. Exact solutions for chemical bond orientations from residual dipolar couplings. J. Biomol. NMR, 22:137-151, 2002.

[164] A. C. Fowler, F. Tian, H. M. Al-Hashimi, and J. H. Prestegard. Rapid determination of protein folds using residual dipolar couplings. J. Mol. Biol., 304(3):447-460, 2000.

[165] L. Wang and B. R. Donald. An efficient and accurate algorithm for assigning nuclear overhauser effect restraints using a rotamer library ensemble and residual dipolar couplings. In IEEE Computer Society Bioinformatics Conference, pages 189202, Stanford University, CA, 2005.

[166] L. Wang and B. R. Donald. An algebraic geometry approach to backbone structure determination from NMR data. In IEEE Computer Society Bioinformatics Conference, pages 235-246, Stanford University, CA, 2005.

[167] Y. Qu, J. T Guo, V. Olman, and Xu Y. Protein fold recognition using residual dipolar coupling data. Nucleic Acids Research, 32:551-561, 2004.

[168] J. Meiler, W. Peti, and C. Griesinger. Dipocoup: a versatile program for 3d-structure homology comparison based on residual dipolar couplings and pseudocontact shifts. J. Biomol. NMR, 17:283-294, 2000.

[169] A. Annila, H. Aitio, E. Thulin, and T. Drakenberg. Recognition of protein folds via dipolar couplings. J. Biomol. NMR, 14:223-230, 1999.

[170] M. Andrec, P. Du, and R. M. Levy. Protein structural motif recognition via NMR residual dipolar couplings. J. Am. Chem. Soc., 123:1222-1229, 2001.

[171] C. Langmead and B. R. Donald. High-throughput 3D structural homology detection via NMR resonance assignment. In IEEE Computer Society Bioinformatics Conference, pages 278-289, Stanford University, CA, 2004.

[172] C. Tanford. Protein denaturation. part C. theoretical models for the mechanism of denaturation. Adv. Protein Chem., 24:1-95, 1970.

[173] N. C. Fitzkee and G. D. Rose. Reassessing random-coil statistics in unfolded proteins. Proc. Natl. Acad. Sci. USA, 101:1249712502, 2004.

[174] A. K. Jha, A. Colubri, K. F. Freed, and T. R. Sosnick. Statistical coil model of the unfolded state: Resolving the reconciliation problem. Proc. Natl. Acad. Sci. USA, 102:13099-13104, 2005.

[175] P. J. Plory. Statistical Mechanics of Chain Molecules. Oxford University Press, New York, 1988.

[176] I. S. Millett, S. Doniach, and K. W. Plaxco. Toward a taxonomy of the denatured state: small angle scattering studies of unfolded proteins. Adv. Protein Chem., 62:241-262, 2002.

[177] D. Shortle and M. S. Ackerman. Persistence of native-like topology in a denatured protein in $8 \mathrm{~m}$ urea. Science, 293:487-489, 2001.

[178] S. Ohnishi, A. L. Lee, M. H. Edgell, and D. Shortle. Direct demonstration of structural similarity between native and denatured eglin c. Biochemistry, 43:4064-4070, 2004. 
[179] S. Fieber, W. Kristjansdottir and F. M. Poulsen. Short-range, long-range and transition state interactions in the denatured state of acbp from residual dipolar couplings. J. Mol. Biol., 339:1191-1199, 2004.

[180] M. Louhivuori, K. Paakkonen, K. Fredriksson, P. Permi, J. Lounila, and A. Annila. On the origin of residual dipolar couplings from denatured proteins. J. Am. Chem. Soc., 125:15647-15650, 2003.

[181] J. M. Ding, K. Louis and A. M. Gronenborn. Insights into conformation and dynamics of protein GB1 during folding and unfolding by NMR. J. Mol. Biol., 335:1299-1307, 2004.

[182] R. Mohana-Borges, N. K. Goto, G. J. Kroon, H. J. Dyson, and P. E. Wright. Structural characterization of unfolded states of apomyoglobin using residual dipolar couplings. J. Mol. Biol., 340:1131-1142, 2004.

[183] M. S. Ackerman and D. Shortle. Molecular alignment of denatured states of staphylococcal nuclease with strained polyacrylamide gels and surfactant liquid crystalline phases. Biochemistry, 41:3089-3095, 2002.

[184] H. J. Sass, G. Musco, S. J. Stahl, P. T. Wingfield, and S. Grzesiek. Solution NMR of proteins within polyacrylamide gels: diffusional properties and residual alignment by mechanical stress or embedding of oriented purple membranes. J. Biomol. NMR, 18:303-309, 2000.

[185] W. Feller. An Introduction to Probability Theory and Its Applications. John Wiley and Sons, Inc, New York, 1970.

[186] S. Chandrasekhar. Stochastic problems in physics and astronomy. Rev. Mod. Phys., 15:1-89, 1943.

[187] L. Wang and H. Yan. NMR studies of type II human cellular retinoic acid binding protein. Biochimica et Biophysica Acta, 1433:240-252, 1999.

[188] L. Wang, Y. Pang, T. Holder, J. Brender, A. V. Kurochkin, and E. R. P. Zuiderweg. Functional dynamics in the active site of the ribonuclease binase. Proc. Natl. Acad. Sci. USA, 98:7684-7689, 2001.

[189] F. Tian, J. L. Al-Hashimi, H. M. Craighead, and J. H. Prestegard. Conformational analysis of a flexible oligosaccharide using residual dipolar couplings. J. Am. Chem. Soc., 123:485-492, 2001.

[190] B. W. Koenig, G. Kontaxis, D. C. Mitchell, J. M. Louis, B. J. Litman, and A. Bax. Structure and orientation of a g protein fragment in the receptor bound state from residual dipolar couplings. J. Mol. Biol., 322:441-461, 2002.

[191] H. M. Bolon, P. J. Al-Hashimi and J. H. Prestegard. Residual dipolar coupling derived orientational constraints on ligand geometry in a 53 kDa protein-ligand complex. J. Mol. Biol., 293:107-115, 1999.

[192] K. Umemoto, H. Leffler, A. Venot, H. Valafar, and J. H. Prestegard. Conformational differences in liganded and unliganded states of Galectin-3. Biochemistry, 42:3688-3695, 2003.

[193] E. R. Zuiderweg. Mapping protein-protein interactions in solution by NMR spectroscopy. Biochemistry, 41:1-7, 2002.

[194] M. W. Fischer, J. A. Losonczi, J. L. Weaver, and J. H. Prestegard. Domain orientation and dynamics in multidomain proteins from residual dipolar couplings. Biochemistry, 38:9013-9022, 1999.

[195] A. T. Brünger. XPLOR: A system for X-ray crystallography and NMR. Yale University Press: New Haven, 1993.

[196] J. A. Lukin, G. Kontaxis, V. Simplaceanu, Y. Yuan, A Bax, and C. Ho. Quaternary structure of hemoglobin in solution. Proc. Natl. Acad. Sci. USA, 100:517-520, 2003.

[197] Y. Zhang and E. R. Zuiderweg. The 70-kDa heat shock protein chaperone nucleotide-binding domain in solution unveiled as a molecular machine that can reorient its functional subdomains. Proc. Natl. Acad. Sci. USA, 101:10272-10277, 2004.

[198] P. Dosset, J. C. Hus, D. Marion, and M. Blackledge. A novel interactive tool for rigid-body modeling of multi-domain macromolecules using residual dipolar couplings. J. Biomol. NMR., 20:223-31, 2001.

[199] D. S. Garrett, Y. J. Seok, A. Peterkofsky, A. M. Gronenborn, and G. M. Clore. Solution structure of the 40,000 Mr phosphoryl transfer complex between the N-terminal domain of enzyme I and HPr. Nat. Struct. Biol., 6:166-173, 1999.

[200] G. M. Clore and C. A. Bewley. Using conjoined rigid body/torsion angle simulated annealing to determine the relative orientation of covalently linked protein domains from dipolar couplings. J. Magn. Reson., 154:329-335, 2002.

[201] G. Cornilescu, B. R. Lee, C. C. Cornilescu, G. Wang, A. Peterkofsky, and G. M. Clore. Solution structure of the phosphoryl transfer complex between the cytoplasmic a domain of the mannitol transporter II mannitol and HPr of the Escherichia coli phosphotransferase system. J. Biol. Chem., 277:42289-42298, 2002.

[202] N. U. Jain, T. J. Wyckoff, C. R. Raetz, and J. H. Prestegard. Rapid analysis of large protein-protein complexes using nmr-derived orientational constraints: the $95 \mathrm{kda}$ complex of lpxa with acyl carrier protein. J. Mol. Biol., 343:1379-1389, 2004.

[203] C. Dominguez, R. Boelens, and A. M. J. J. Bonvin. HADDOCK: A protein-protein docking approach based on biochemical or biophysical information. J. Am. Chem. Soc., 125:1731-1737, 2003.

[204] M. A. McCoy and D. F. Wyss. Structures of protein-protein complexes are docked using only nmr restraints from residual dipolar coupling and chemical shift perturbations. J. Am. Chem. Soc., 124:2104-2105, 2002. 
[205] Dobrodumov A and Gronenborn AM. Filtering and selection of structural models: combining docking and NMR. Proteins, 53:18-32, 2003.

[206] H. A. Gabb, R. M. Jackson, and M. J. E. Sternberg. Modelling protein docking using shape complimentarity, electrostatics, and biochemical information. J. Mol. Biol., 272:106-120, 1997.

[207] T. Matsuda, T. Ikegami, N. Nakajima, T. Yamazaki, and H. Nakamura. Model building of a protein-protein complexed structure using saturation transfer and residual dipolar coupling without paired intermolecular noe. J. Biomol. NMR., 29:325-328, 2004. 